University of Louisville

ThinkIR: The University of Louisville's Institutional Repository

Electronic Theses and Dissertations

8-2017

\title{
UBQLN1 : a multi-domain protein with multiple functions.
}

Zimple Kurlawala

University of Louisville

Follow this and additional works at: https://ir.library.louisville.edu/etd

Part of the Biological Phenomena, Cell Phenomena, and Immunity Commons, Medical Cell Biology Commons, Medical Molecular Biology Commons, and the Physiological Processes Commons

\section{Recommended Citation}

Kurlawala, Zimple, "UBQLN1 : a multi-domain protein with multiple functions." (2017). Electronic Theses and Dissertations. Paper 2754.

https://doi.org/10.18297/etd/2754

This Doctoral Dissertation is brought to you for free and open access by ThinkIR: The University of Louisville's Institutional Repository. It has been accepted for inclusion in Electronic Theses and Dissertations by an authorized administrator of ThinkIR: The University of Louisville's Institutional Repository. This title appears here courtesy of the author, who has retained all other copyrights. For more information, please contact thinkir@louisville.edu. 
UBQLN1: A MULTI-DOMAIN PROTEIN WITH MULTIPLE FUNCTIONS

By

\author{
Zimple Kurlawala \\ M.D., M.P.H., M.S.

\begin{abstract}
A Dissertation
Submitted to the Faculty of

School of Medicine at University of Louisville

In Partial Fulfillment of the Requirements For the Degree of
\end{abstract}

\author{
Doctor of Philosophy \\ in Pharmacology and Toxicology \\ Department of Pharmacology and Toxicology \\ University of Louisville \\ Louisville, $\mathrm{KY}$
}

August 2017 
Copyright 2017

By

Zimple Kurlawala

All rights reserved 

UBQLN1: A MULTI-DOMAIN PROTEIN WITH MULTPLE FUNCTIONS By

Zimple Kurlawala

M.S. Pharmacology and Toxicology, University of Louisville, 2015 M.P.H Western Kentucky University, 2011

M.D. University of Seychelles-American Institute of Medicine, 2008

Dissertation Approved on July $30^{\text {th }}, 2017$ by

Following Dissertation Committee

Levi Beverly

Leah Siskind

Brian Ceresa

Geoff CLark

Jesse Roman 


\section{DEDICATION}

I dedicate this PhD dissertation to my parents, Dinesh Kurlawala and Hemlata Kurlawala. Without their love, endless support and sacrifices, it would have been impossible for me to achieve this feat. 


\section{ACKNOWLEDGEMENTS}

The four years I spent working towards my $\mathrm{PhD}$ in the Beverly lab have been the most inspirational in my life. For this and for introducing me to the world of scientific research, I thank Dr. Levi Beverly.

I would like to thank Dr. Brian Ceresa for sharing with us his expertise on receptor biology. His attention to detail and proficiency with various pharmacological techniques have influenced me tremendously. This $\mathrm{PhD}$ Dissertation would be incomplete without Dr. Ceresa's inputs.

I would like to express my gratitude towards Dr. Leah Siskind for her guidance and for being a "hype-man" for all lab members whenever we need it. I would also like to thank Leah for her generosity in loaning me her lab desktop and the use of her office for dissertation writing.

I would also like to thank Dr. Geoffery Clark and Dr. Jesse Roman for their invaluable inputs to my project.

I would like to thank all members of the Beverly and Siskind labs for their help, support and friendship, especially Lavona Casson, Mark Doll, Cierra Sharp, Parag Shah and Subathra Marimuthu. Special thanks to Doug Saforo and Zach Sellers for being excellent friends and to Rain Dunaway aka "Draino" for giving me the opportunity to mentor her and for assisting me in completing my dissertation. 


\section{ABSTRACT \\ UBQLN1: A MULTI-DOMAIN PROTEIN WITH MULTPLE FUNCTIONS \\ Zimple Kurlawala}

July 25,2017

There are 5 Ubiquilin proteins (UBQLN1-4, UBQLN-L), which are evolutionarily conserved and structurally similar. UBQLN proteins have 3 functional domains: $\mathrm{N}$ terminal ubiquitin-like domain (UBL), C-terminal ubiquitin-associated domain (UBA) and STI chaperone-like regions in the middle. Alterations in UBQLN1 gene have been detected in a variety of disorders including Alzheimer's disease, Amyotropic Lateral Sclerosis and lung cancer. UBQLN1 has been largely studied in neurodegenerative disorders in the context of protein quality control. Several studies have hypothesized that the UBA domain of UBQLN1 binds to poly-ubiquitin chains of substrate and shuttles it to the proteasome via its UBL domain for degradation. UBQLN1 can both facilitate degradation (Ataxin3, EPS15) and stabilize (PSEN1/2, BCLb) substrates it binds. The signal that determines this fate is unknown and there is conflicting data to support the existing working model of UBQLN1.

BCLb is a member of BCL2 family of proteins that maintain the balance between apoptosis and survival in cells. BCLb is anti-apoptotic and interacts with UBQLN1. Using BCLb as a model substrate, we characterized UBQLN1-substrate interaction. We identified the first two STI domains of UBQLN1 as critical for binding of the UBA domain to these proteins slows their degradation. Similarly, we showed that UBQLN1 interacts with IGF1R and ESYT2 through the STI domains and binding stabilizes these proteins through its UBA domain. Interactions that are not dependent on STI domains, for example UBL mediated interaction with PSMD4 and BAG6, do not 
appear to be stabilized by UBQLN1. We conclude that fate of substrates that UBQLN associates with, is interaction domain specific. We used data derived from UBQLN1BCLb interactions to model how UBQLN1 regulates IGF1R in lung adenocarcinoma cells. We have identified Ubiquilin1 as a novel interaction partner of IGF1R, IGF2R and Insulin Receptor. We demonstrate here that UBQLN1 regulates expression and activity of IGF receptors. Following loss of UBQLN1 in lung adenocarcinoma cells, there is accelerated loss of IGF1R post stimulation with ligand. Despite decreased levels of total receptors, the ratio of active:total receptors is higher in cells that lack UBQLN1. We tested for differences in synthesis, degradation, trafficking, autocrine ligand production, survival, migration potential and response to chemotherapy in lung adenocarcinoma cells that have loss of UBQLN1. UBQLN1 also regulates ligand stimulated IGF2R, Insulin Receptor (INSR), Epidermal Growth Factor Receptor (EGFR). UBQLN1 deficient cells demonstrate increased survival when serum starved and stimulation of IGF pathway in these cells increased their migratory potential by 3fold. In conclusion, UBQLN1 is essential for normal regulation of these receptor tyrosine kinases as UBQLN1 negatively regulates total receptor levels. 


\section{TABLE OF CONTENTS}

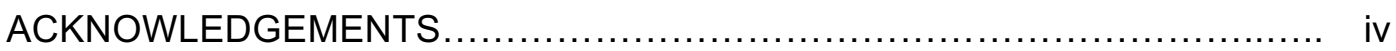

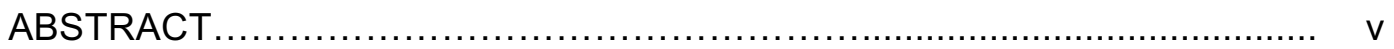

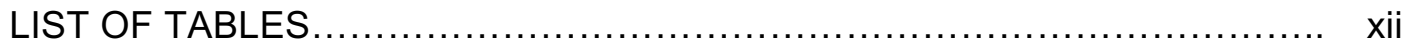

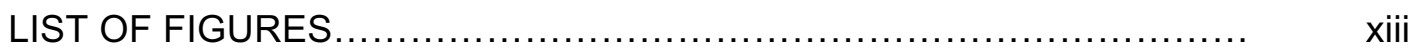

\section{CHAPTER 1}

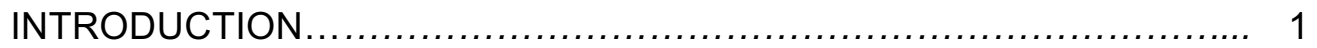

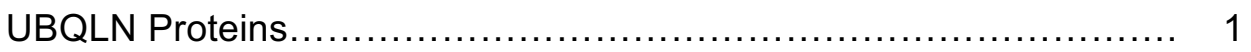

UBQLN and Ubiquitin .............................................. 4

UBQLN and Cell Surface Receptors............................... 6

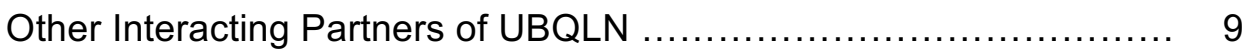

UBQLN in Neurodegenerative Disorders.......................... 10

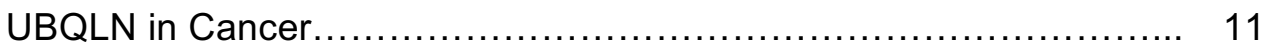

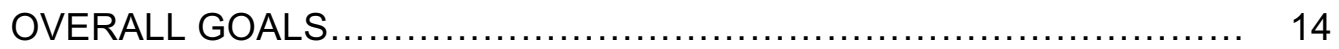

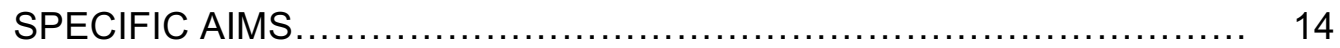

\section{CHAPTER 2}

MATERIALS AND METHODS 15

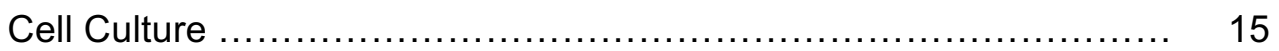

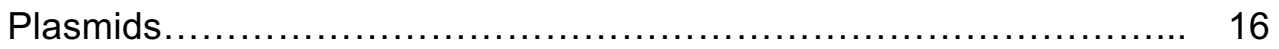

Immunoprecipitation/Western Blot Analysis....................... 17

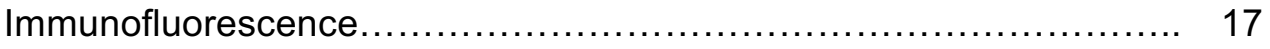

Radioligand Saturation Binding Analysis ........................... 18 
Percoll Density Gradient Centrifugation.

Survival Assay

Transwell Migration

Total RNA Extraction and Real Time PCR.

Antibodies.

RNAi sequences.

INTRODUCTION

RESULTS.

Cellular distribution of UBQLN1

UBQLN1 non-selectively interacts with diverse ubiquitin linkages through its UBA domain.

Recognition of the transmembrane domain of BCLb by STI-1/2 domains of UBQLN1 sequesters BCLb in the cytosol.

Interaction of UBQLN1 with BCLb is independent of ubiquitination of BCLb

UBQLN1 stabilizes BCLb ${ }^{\mathrm{WT}}$ after translational and proteasomal inhibition

IGF1R, ESYT2, and BAG6 are newly identified substrates of UBQLN1. Interaction of UBQLN1 with IGF1R and ESYT2 is STImediated while BAG6 and PSMD4 is UBL-mediated. UBQLN1 is capable of homodimerization through its STI-4 domains 
INTRODUCTION.

Insulin-Like Growth Factor Receptors.

RESULTS

UBQLN1 interacts with IGF1R, IGF2R, and INSR

56

UBQLN1 interacts with pro-form, phosphorylated and non-

phosphorylated forms of IGF1R

UBQLN1 regulates expression and activity of IGF1R in lung

adenocarcinoma cells.

Loss of UBQLN1 results in decreased cell surface expression of IGF1R

Loss of UBQLN1 accelerates loss of IGF1R.

Loss of UBQLN1 inhibits recycling of IGF1R

UBQLN1 does not play a role in IGF1R turnover through the proteasome

UBQLN1 may direct IGF1R towards higher density compartments

UBQLN1 deficient A549 cells have increased survival in serum-free conditions.

Activation of IGF pathway in UBQLN1 deficient cells increases their migration potential 3-fold. 
STI-1/2 domains of UBQLN1 interact with Insulin receptor (INSR) and loss of UBQLN1 regulates expression of INSR and IGF2R poststimulation with IGF1

UBQLN1 deficient A549 cells have decreased autocrine ligand production compared to control

UBQLN1 does not stably associate ith these markers of endocytic trafficking: GRB10, EEA1, RAB7, LAMP1, Caveolin1

Combined loss of UBQLN1 and PTP1B has a synergistic effect on activation of kinase domain of IGF1R

UBQLN1 and UBQLN2 interact with Epidermal Growth Factor Receptor (EGFR).

UBQLN1 regulates EGFR expression in K-RAS mutant A549 cells but not in EGFR mutant $\mathrm{H} 1650$ cells

EGF-dose dependent regulation of EGFR by UBQLN1 ............. 91

DISCUSSION.....

\section{CHAPTER 5}

OVERALL SUMMARY

Overall Goals and Specific Aims.

Major Findings of this Dissertation

Strengths of this Dissertation.

Limitations of this Dissertation

Future Directions

Conclusions. 


\section{LIST OF TABLES}

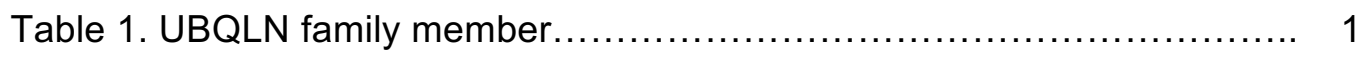

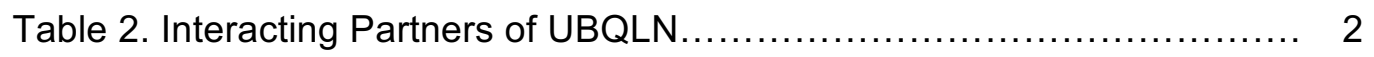




\section{LIST OF FIGURES}

Figure 1. Schematic of structural domains of UBQLN1 .............................. 1

Figure 2. Cellular Distribution of UBQLN1 .................................................. 26

Figure 3. UBQLN1 interacts non-preferentially with diverse ubiquitin linkages through its UBA domain.

Figure 4. Recognition of the transmembrane domain of BCLb by STI-1/2 domains of UBQLN1 sequesters BCLb in the cytosol

Figure 5. Interaction of UBQLN1 with BCLb is independent of ubiquitination of BCLb.

Figure 6. Schematic of mechanism of stabilization of BCLb by UBQLN1.........

Figure 7. UBA domain of UBQLN1 is responsible for stabilization of BCLb ${ }^{\mathrm{WT}} \ldots$

Figure 8. Fate of substrates targeted by UBQLN1 is dependent on interaction of the substrate with specific domains of UBQLN1

Figure 9. UBQLN1 is capable of homodimerization via its STI-4 domains.

Figure 10. Schematic showing substrate domains

Figure 11. Current model vs Proposed model ............................... 45

Figure 12. Models of interaction of UBQLN1 ...................................... 47

Figure 13. Schematic of structural domains of IGF1R................................. 53

Figure 14. IGF1R signaling pathways..................................................... 53

Figure 15. IGF1R structure-function relationship................................... 54

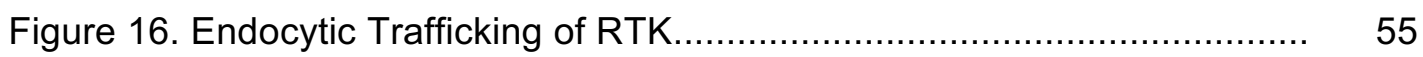

Figure 17. Schematic of clathrin mediated endocytosis of EGFR................. 55

Figure 18. UBQLN1 interacts with IGF1R .............................................. 5 
Figure 19. UBQLN1 interacts with pro-form, phosphorylated and nonphosphorylated forms of IGF1R.

Figure 20. UBQLN1 regulates expression and activity of IGF1R

Figure 21. Loss of UBQLN1 results in decreased cell surface expression of IGF1R

Figure 22. Loss of UBQLN1 accelerates loss of IGF1R

Figure 23. Loss of UBQLN1 inhibits recycling of IGF1R

Figure 24. UBQLN1 does not play a role in IGF1R turnover though the proteasome

Figure 25. Loss of UBQLN1 may direct IGF1R towards higher density compartments

Figure 26. UBQLN1 deficient A549 cells have increased survival in serumfree conditions.

Figure 27. Activation of IGF1 pathway in UBQN1 deficient A549 cells increases their migration potential 3-fold.

Figure 28. STI-1/2 domains of UBQLN1 interact with Insulin receptor (INSR)

Figure 29 Loss of UBQLN1 regulates expression of INSR and IGF2R poststimulation with IGF1

Figure 30. UBQLN1 deficient A549 cells have decreased autocrine ligand production compared to control

Figure 31. UBQLN1 does not stably associate with these markers of endocytic trafficking: GRB10, EEA1, RAB7, LAMP1, Caveolin1....

Figure 32. Combined loss of UBQLN1 and PTP1B has a synergistic effect on activation of kinase domain of IGF1R.

Figure 33. UBQLN1 and UBQLN2 interact with EGFR. 
Figure 34. UBQLN1 regulates EGFR expression in K-RAS mutant A549 cells but not in EGFR mutant H1650 cells.................. 90

Figure 35. EGF-dose dependent regulation of EGFR by UBQLN1 ............... 92

Figure 36. Model of regulation of IGF1R by UBQLN1 ........... 99 


\section{CHAPTER I}

\section{INTRODUCTION}

\section{UBQLN Proteins}

Ubiquilin proteins are implicated in a variety of diseases like Alzheimer's disease, Amyotropic Lateral Sclerosis, Fronto-Temporal dementia, triple repeat pathologies and even in lung, bone and urothelial cancers. How loss of function of a protein can lead to diverse outcomes

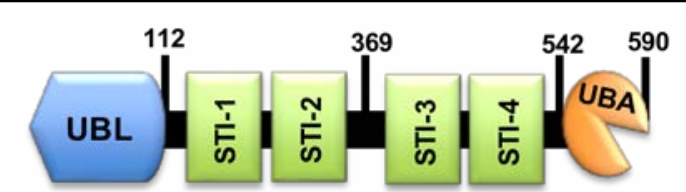

Figure 1: Schematic of structural domains of UBQLN1. UBL domain: UBiquitin-Like domain. STI-1,2,3,4 domains: STress-Inducible proteins. These domains mediate protein-protein interactions. UBA domain: UBiquitinAssociated domain associates with ubiquitin.

in different cell types is intriguing. For this dissertation, we were interested

understanding in mechanism of action of UBQLN1 and how it relates to differential regulation of its substrates.

The Ubiquilin
family of proteins (UBQLN1-4, UBQLNL) are evolutionarily

\begin{tabular}{|c|c|c|c|c|}
\hline Gene & Location & $\begin{array}{l}\text { Size } \\
\text { (amino } \\
\text { acids) }\end{array}$ & $\begin{array}{c}\text { Sequence } \\
\text { similarity } \\
\text { to } \\
\text { UBQLN1 } \\
(\%)\end{array}$ & $\begin{array}{c}\text { Tissue } \\
\text { Distribution }\end{array}$ \\
\hline UBQLN1 & $9 q 21.32$ & 589 & 100 & All \\
\hline UBQLN2 & Xp11.21 & 624 & 74 & $\begin{array}{c}\text { Brain, } \\
\text { spleen, } \\
\text { heart, liver } \\
\text { and } \\
\text { pancreas }\end{array}$ \\
\hline UBQLN3 & $11 \mathrm{p} 15.4$ & 655 & 48 & Testis \\
\hline UBQLN4 & $1 q 22$ & 601 & 60 & $\begin{array}{c}\text { Brain, } \\
\text { spleen, } \\
\text { heart, liver } \\
\text { and } \\
\text { pancreas }\end{array}$ \\
\hline UBQLNL & 11 & 475 & 36 & $\begin{array}{l}\text { Plasma, } \\
\text { platelet and } \\
\text { liver }\end{array}$ \\
\hline
\end{tabular}

Table 1: UBQLN family members, their chromosomal location and tissue distribution. Percent of sequence similarity is in comparison to sequence of UBQLN1. 


\begin{tabular}{|c|c|c|}
\hline \multicolumn{3}{|c|}{ Table 2: Interacting partners of UBQLN } \\
\hline & $\begin{array}{l}\text { Interacting Partner } \\
\end{array}$ & Mapped Domains \\
\hline \multicolumn{3}{|c|}{ Cell Surface Receptors } \\
\hline 1 & $\begin{array}{l}\text { IAP(A. L. Wu, Wang, Zheleznyak, \& Brown, } \\
\text { 1999) }\end{array}$ & UBL \\
\hline 2 & $\begin{array}{l}\begin{array}{l}\text { Presinilin1 and 2(Mah, Perry, Smith, \& } \\
\text { Monteiro, 2000) }\end{array} \\
\end{array}$ & $\begin{array}{l}\text { UBA and } \mathrm{COOH} \text { tail } \\
\text { mediated. }\end{array}$ \\
\hline 3 & $\mathrm{GABA}_{\mathrm{A}}$ (Bedford et al., 2001) & $\begin{array}{l}\text { UBA } \\
\left(\text { GABA }_{A} \text { : between Tm }\right. \\
\text { domains } 3 \text { and } 4 \text { of the } \\
\text { alpha1 subunit) }\end{array}$ \\
\hline 4 & $\begin{array}{l}\text { IGF1R (Kurlawala, Shah, Shah, \& Beverly, } \\
\text { 2017) INSR }\end{array}$ & STI-1 and STI-2 \\
\hline 5 & GPCR's (N'Diaye et al., 2008) & Not mapped \\
\hline 6 & nAChR(Ficklin, Zhao, \& Feng, 2005) & Not mapped \\
\hline 7 & ESYT2 (Kurlawala et al., 2017) & STI-1 and STI-2 \\
\hline \multicolumn{3}{|c|}{ Proteasomal Machinery } \\
\hline 8 & $\begin{array}{l}\text { S5a (Rpn10a), (Ko, Uehara, Tsuruma, \& } \\
\text { Nomura, 2004) Rpn3, Rpn10e }\end{array}$ & UBL \\
\hline 9 & PSMC2 (Muller-Vahl, Kolbe, \& Dengler, 1997) & Not mapped \\
\hline \multicolumn{3}{|c|}{ Transmembrane Proteins } \\
\hline 10 & K7(Feng et al., 2004) & UBA \\
\hline 11 & Omp25 (Itakura et al., 2016) & Not mapped \\
\hline 12 & 12 Mtb proteins (Sakowski et al., 2015) & Not mapped \\
\hline 13 & $\begin{array}{l}\text { BCLb (Beverly, Lockwood, Shah, Erdjument- } \\
\text { Bromage, \& Varmus, 2012; Kurlawala et al., } \\
\text { 2017) }\end{array}$ & STI-1 and STI-2 \\
\hline \multicolumn{3}{|c|}{ Others } \\
\hline 14 & $\begin{array}{l}\text { Ubiquitin(Feng et al., 2004; Ko, Uehara, \& } \\
\text { Nomura, 2002; Kurlawala et al., 2017; Massey } \\
\text { et al., 2004; D. Zhang, Raasi, \& Fushman, } \\
\text { 2008) }\end{array}$ & \\
\hline 15 & $\begin{array}{l}\text { HERC3 (Hochrainer, Kroismayr, Baranyi, } \\
\text { Binder, \& Lipp, 2008) }\end{array}$ & Not mapped \\
\hline 16 & $\begin{array}{l}\text { EPS15, EPS15R, Hrs, Hbp (Regan-Klapisz et } \\
\text { al., 2005) }\end{array}$ & UBL \\
\hline 17 & $\begin{array}{l}\text { UBQLN1 homodimer (Feng et al., 2004; } \\
\text { Kurlawala et al., 2017) } \\
\text { UBQLN2 heterodimer (Feng et al., 2004; } \\
\text { Hjerpe et al., 2016) }\end{array}$ & STI-4 (homodimer) \\
\hline 18 & mTOR (S. Wu et al., 2002) & $\begin{array}{l}\text { STI-1 and STI-2 (226- } \\
323)\end{array}$ \\
\hline 19 & $\begin{array}{l}\text { E6AP, several proteasomal proteins (Kleijnen } \\
\text { et al., 2000) }\end{array}$ & Not mapped \\
\hline 20 & PDI (Ko et al., 2002) & Not mapped \\
\hline
\end{tabular}


conserved and structurally similar to each other. These proteins are highly similar in sequence identity, encoded by genes on different chromosomes and have varied tissue distribution (Table 1) (Beverly et al., 2012). UBQLN1 is approximately $63 \mathrm{kDa}$ protein and all its family members have 3 main domains: ubiquitin-like domain (UBL) at the $\mathrm{N}$-terminus, ubiquitin-associated domain (UBA) at the C-terminus and STI chaperone-like regions in the middle (Fig. 1). UBQLN1 maintains all the functionally important motifs present in this group (Massey et al., 2004) is ubiquitously expressed in all tissues. The UBL domain of UBQLN is structurally similar to ubiquitin and interacts proteasomal cap (S5a) (Buchberger, 2002) while the UBA domain associates with ubiquitin (Wilkinson et al., 2001; D. Zhang et al., 2008).

The central region consisting of STI domains are called so because of their similarity to STI-1 proteins (STress Inducible proteins or Hsp70-Hsp90 organizing protein, HOP). This region acts as a co-chaperone and mediates hydrophobic interactions with other proteins (Mueller, Kamionka, \& Feigon, 2004; L. Zhao \& Ackerman, 2006). Current working model of UBQLN1 is hypothesized as: the UBL domain of Ubiquilin1 binds to the proteasome while the UBA domain binds to ubiquitin on the substrate protein and aids in the substrate's degradation (Buchberger, 2002).

There are 2 types of UBL-UBA proteins: type-1 like SUMO and NEDD1 which covalently attach to ubiquitin and type-2 proteins like UBQLN, Rad23, Parkin, Bat-3, Elongin-b, Bag-1 and so on that associate with ubiquitin through its UBA domain in a non-covalent manner. As a type-2 UBL-UBA protein, UBQLN proteins are mostly studied in the context of ubiquitination, protein degradation and protein quality control and are largely implicated in development of neurodegenerative disorders. Table 1 shows a list of published substrates of UBQLN1. Ubiquilin1 interacts with a variety of proteins - involved in the proteasomal machinery (PSMD4, BAG6, S5a, Rpn3, Rpn10e) (Kurlawala et al., 2017), cell surface receptors (IAP, GABA-A (Saliba, Pangalos, \& Moss, 2008) , GPCR's (N'Diaye et al., 2008), Presinilins 1/2 (Mah et al., 2000; Massey et al., 2004), IGF1R (Kurlawala et al., 2017), transcription factor 
regulators (IKBa) (Feng et al., 2004) and intracellular transmembrane proteins ESYT2 (Kurlawala et al., 2017), CD47 (A. L. Wu et al., 1999), BCLb (Beverly et al., 2012), OMP25 (Itakura et al., 2016), and others like ubiquitin, E3 ligases and so on. Additionally, Ubiquilin1 function is implicated in diverse cellular processes like ERAD (endoplasmic reticulum associated degradation) (Lim et al., 2009; Shah et al., 2015), autophagy (Lee, Arnott, \& Brown, 2013; Elsa-Noah N'Diaye et al., 2009; Sun et al., 2015), apoptosis (Lu et al., 2009) and epithelial to mesenchymal transition (EMT) (Shah et al., 2015; Yadav et al., 2017).

\section{UBQLN1 and Ubiquitin}

The most well understood interacting partner of UBQLN proteins is ubiquitin. Ubiquitin is a $7 \mathrm{kD}$ molecule that covalently binds to lysine residues on proteins. One ubiquitin molecule has 7 lysine residues (K6, K11, K27, K29, K33, K48, K63). Ubiquitin is capable of mono-ubiquitinating proteins and forming ubiquitin chains through one or more of its 7 lysines. The process of ubiquitination involves 3 ubiquitin ligases E1 (ubiquitin-activating enzyme), E2 (ubiquitin-conjugating enzyme) and E3, which recruits an E2 ubiquitin-conjugating enzyme that has been loaded with ubiquitin, recognizes a protein substrate, and assists in or directly catalyzes the transfer of ubiquitin from the E2 ligase to the protein substrate.

Ubiquilin associates non-preferentially with mono- and poly-ubiquitin linkages through its UBA domain (Feng et al., 2004; Ko et al., 2002; Kurlawala et al., 2017; Massey et al., 2004; D. Zhang et al., 2008). The most widely accepted working model of UBQLN1 suggests that UBQLN1 associates with ubiquitin on its substrates through its UBA domain, while the UBL domain (known interactor of the proteasome) directs it for degradation. However, UBA domain of UBQLN1 can act as a receptor for ubiquitin on substrates, and shield them from degradation thus stabilizing the substrate. It is certain that UBA domain binds ubiquitin but there is no consensus on fate of interaction 
of Ubiquilin1 with its substrate. Interestingly, Ubiquilin1 is also reported to interact with three E3 ligases -- E6AP and $\beta^{\text {TRCP }}$ (Kleijnen et al., 2000)' and HERC3 (Hochrainer et al., 2008). Upon interaction with E6AP and $\beta^{\text {TRCP }}$, UBQLN1 and UBQLN2 prevent degradation of their substrates - tumor suppressor p53 and TNF $\alpha$-induced rapid degradation of inflammatory mediator $I_{\kappa} B \alpha$, respectively. Overexpression of UBQLN proteins did not block the proteasome as the control protein Ornithine Decarboxylase, a known substrate of the proteasome continued to be degraded at its usual rate. Therefore, it is likely that UBQLN1 interaction with E3 ligases prevents a step that is common to both pathways in the presence of overexpressed UBQLN's. In contrast, Hochrainer et al showed that UBQLN1 interacts with HERC3, another E3 ligase and stabilizes HERC3's interaction with proteasome and NFKB. UBQLN1 strengthens binding of HERC3 to ReIA, which delivers RelA to proteasome and thus has a degradation enhancing role for NFאB (Hochrainer et al., 2008; Hochrainer et al., 2015). Therefore, it strengthens the hypothesis that UBQLN1 does have substrate specific functions.

\section{UBQLN and Cell Surface Receptors}

The largest group of known substrates of UBQLN proteins are cell surface receptors, namely CD47, Presinilins, GABA $A_{A}$ IGF1R, GPCR's. Here, we'll review the ways Ubiquilin regulates these proteins.

Ubiquilins were originally discovered as Proteins Linking IAP with Cytoskeleton (PLICs) by Wu Ai-Ling in 1999 as proteins that interact with CD47 (integrin associated protein, IAP) that anchor vimentin filaments to the plasma membrane. UBQLN1 and UBQLN2 were distribution was contained in the cytoplasm and membranes of adherent, suspension as well as cytochalasin D (inhibitor of actin polymerization) treated cells (A. L. Wu et al., 1999) and their overexpression increased cell spreading in ovarian cancer cells. 
Soon after, Presenilin1 and Presinilin2 were identified as new interacting partners of UBQLN1 (Mah et al., 2000) and this discovery was of particular interest in the 1990's since mutations in familial cases of Alzheimer's had recently been identified in Presinilin and APP genes. Overexpression of UBQLN1 in HeLa cells led to increased expression (number of receptors) of Presenilins without increase in synthesis or altering protein degradation. This indicates that UBQLN1 may enhance post translational modifications of Presenilins in a way that presence of UBQLN1 prevents Presenilins' untimely loss (Mah et al., 2000; Massey et al., 2004). Additionally, UBQLN1 decreased ubiquitination of Presinilins and as a result decreased its fragmentation in the gamma secretase complex into $\mathrm{N}$-terminal and $\mathrm{C}$ terminal fragments (NTF and CTF). The NTF and CTF fragments of Presenilins form the catalytic core of the gamma secretase complex (Massey, Mah, \& Monteiro, 2005). Both, overexpression of UBQLN1 and blocking of the proteasome with multiple inhibitors inhibited fragmentation of Presinilins. Therefore, UBQLN1 plays a role via the proteasome, either directly or indirectly, in regulating components of the gamma secretase complex. UBQLN1 also effected other components of the gamma secretase complex namely Pen2, Aph-1 and Nicastrin. Overexpression of UBQLN1, increased levels of Pen2 simultaneously as Presenilin fragmentation increased and Aph-1 and mature Nicastrin levels were decreased.

UBQLN1 interacts with another cell surface receptor in neurons, GABA receptors (Bedford et al., 2001). UBQLN1 overexpression enhanced cell surface levels of $\mathrm{GABA}_{\mathrm{A}}$ receptors demonstrated by ELISA (Bedford et al., 2001) and biotinylation assays (Saliba et al., 2008). Overexpression of UBQLN1 increased half-life of GABA receptors and prevented its loss upon proteasomal inhibition. Disruption of interaction between $U B Q L N 1$ with $\mathrm{GABA}_{A}$ receptors produced a rapid decrease in its cell surface receptor expression within minutes of loss of interaction, suggesting that UBQLN1 may play a role in maintaining anchorage of $\mathrm{GABA}_{A}$ in the plasma membrane. Additionally, immunofluorescence localized UBQLN1 to GABAergic synapses in clathrin-coated 
vesicles, intracellular membranes and inhibitory synapses suggesting that UBQLN1 may also play a role in stabilizing both recycled and de novo synthesized $G_{A B A}$ receptors. Transmembrane proteins like $\mathrm{GABA}_{A}$ receptors usually require assembly in the Endoplasmic Reticulum once they are translated. These receptors are conjugated with high mannose $\mathrm{N}$-linked glycans in the ER, that are sensitive to cleavage by the enzyme, Endoglycosidase $\mathrm{H}$ (Endo $\mathrm{H}$ ). Endo $\mathrm{H}$ enzyme is commonly used to study post translational modifications by specifically removing high mannose $\mathrm{N}$-linked carbohydrates from substrates. Once proteins the ER for Golgi for further processing, they are conjugated with mature $\mathrm{N}$-linked glycans which are insensitive to cleavage by Endo $\mathrm{H}$. Overexpression of UBQLN1 increased the amount of Endo $\mathrm{H}$ sensitive GABA in the ER by $\sim 30 \%$ thus confirming a role for UBQLN1 in stabilizing newly translated transmembrane proteins in the ER (Saliba et al., 2008). Additionally, UBQLN1 also increased ubiquitinated GABA-R, enhanced cell surface accumulation of recombinant GABA beta3 subunits in neurons and increased insertion of $G_{A B A}$ into neuronal plasma membranes.

UBQLN1's UBA domain binds to K7, a small membrane protein in Kaposi Sarcoma Associated Herpes Virus infected cells (Feng et al., 2004) and regulates in similar fashion as it regulates $G A B A_{A}$ receptors. As seen with Presenilins and $G A B A_{A}$ receptors, overexpression of UBQLN1 increased overall expression of $\mathrm{K} 7 . \mathrm{K} 7$ has a half-life of 30 minutes and undergoes proteasomal degradation. Overexpressing UBQLN1 increased half-life of K7 by 12 -fold mediated exclusively by its UBA domain as overexpressing UBA domain by itself and AP1, a UBA domain harboring protein, also prevent loss of $\mathrm{K} 7$ through proteasomal degradation implying that UBQLN1 binds to $\mathrm{K} 7$ through its UBA domain and may be preventing its proteasomal degradation thus stabilizing it (Feng et al., 2004). UBA domain overexpression also prevented loss of p53 and $I_{\kappa} B \alpha$, substrates that are known to be stabilized by UBQLN1 overexpression. Reciprocally, overexpression of $\mathrm{K} 7$ led to decreased ubiquitination and dimerization of 
UBQLN1, and accelerated degradation of $p 53$ and $I_{\kappa} B \alpha$, likely due to occupancy of UBA domain by $\mathrm{K} 7$.

UBQLN1 interacts with the $\alpha 3, \alpha 4$ and $\beta 4$ subunits of nicotinic acetylcholine receptors (nAchR's) in SCG neurons. Overexpression of UBQLN1 prevented nicotineinduced upregulation of cell surface nAchR's assessed by biotinylation assays and immunofluorescence staining (Ficklin et al., 2005). UBQLN1 was identified to bind to 12 cell surface proteins (Mtb's) of Mycobacterium Tuberculosis in macrophages. UBQLN1 enhances ubiquitination of these Mtb's and promoted IFN $\gamma$ driven autophagy to restrict growth of these bacteria (Sakowski et al., 2015).

Overall, UBQLN1 interacts with several cell surface receptors. Overexpression of UBQLN1 stabilizes expression of Presenlins, GABA $A_{A}$ receptors, $K 7$ protein. Although its exact function is not known, it is likely that UBQLN1 functions to anchor receptor complexes in the plasma membrane and prevents their untimely activation and degradation.

\section{Other interacting partners of UBQLN1}

Wu et al 2002, identified and studied interaction of UBQLN1 with the protein kinase, mTOR (S. Wu et al., 2002). Region 226-323 i.e. parts of STI-1 and STI-2 domains of UBQLN1 were mapped to interact with catalytically inactive region of mTOR. By sucrose density gradient fractionations, mTOR was detected with $10 \%$ of total Ubiquilin1 in low density membranes. The biological relevance of interaction between these proteins is known as expression of UBQLN1 did not alter phosphorylation status of this kinase or its targets like p70 (S6 kinase). Hypoxia induced expression of UBQLN1 and an ER enzyme Protein Disulfide Isomerase (PDI) in neuronal cells. Their upregulation and interaction with each other protected neuronal cells from hypoxia induced ER stress and DNA fragmentation (apoptosis) (Ko et al., 2002) . Endocytic proteins like EPS15, EPS15R, Hrs, Hbp harbor ubiquitin interacting 
motifs (UIMs) which are like UBA domains and therefore can interact with ubiquitin or or ubiquitin like domains (UBLs). These endocytic proteins interact with UBQLN1's UBL domain. Overexpression of UBQLN1 causes sequestration of these endocytic proteins into ubiquitin rich cytosolic aggregates (Regan-Klapisz et al., 2005). UBQLN2 was identified to heteromerize with other UBQLN1 (UBQLN1, UBQLN2 and UBQLN4) and its interaction with HSP70 mediates unfolding of substrates and lead to their proteasomal degradation independent of autophagy. Disease mutations of UBQLN2 inhibit this interaction and lead to aggregations and degeneration in neurons (Hjerpe et al., 2016). UBQLN1 can bind transmembrane proteins in the cytosol and prevent them from aggregating. UBQLN was proposed to exist in a closed conformation (interaction of UBL domain of one UBQLN molecule with UBA domain of same or other UBQLN molecules) and supposed to "open" when there is a substrate (Itakura et al., 2016). Dsk2p, the yeast homolog of UBQLN1, forms a trimeric complex with Rad23p and Cdc48 and enables degradation of misfolded proteins via the proteasomal system (Medicherla, Kostova, Schaefer, \& Wolf, 2004). In human cells, the UBL domain of UBQLN1 has been shown to regulate degradation of misfolded proteins like Ataxin3, HSJ1a and EPS15 (Heir et al., 2006). UBQLN1 expression aids in poly-ubiquitination of viral polymerase NS5B, decreasing its half-life in HCV infected hepatocytes (Gao et al., 2003). Identifying functions of Ubiquilin proteins is an ongoing area of research. UBQLN1 is a stress-response protein; its expression protects astrocytes from hypoxia (Ko et al., 2002) and HeLa cells from starvation-induced death (E. N. N'Diaye et al., 2009). In 2010, Rothenberg et al showed that UBQLN1 interacts with autophagy marker LC3 in a complex with other proteins and reduction in UBQLN1 expression decreases autophagy levels in HeLa cells, brain and liver tissue of mice (Rothenberg et al., 2010). 


\section{UBQLN in Neurodegenerative Disorders}

UBQLN1 and UBQLN2 are members of UBQLN family that are primarily implicated in neurodegenerative disorders. UBQLN2 has an extra collagen like domain where majority of ALS mutations are found. UBQLN2 mutations cause defects in autophagy and drive accumulation of TDP43, etc. Presenilin proteins (PS1 and 2) are well-established Alzheimer's disease-associated proteins (Tanzi \& Bertram, 2005). UBQLN1 binds to and stabilizes Presinilin1 and 2 and co-localize in a vesicular-like or ER-like pattern (Gao et al., 2003; Massey et al., 2004). A meta-analysis in 2014 confirmed that a single intronic C/T polymorphism in UBQLN1 (UBQ-8i) significantly increased risk of Alzheimer's disease (Bertram et al., 2005; T. Zhang \& Jia, 2014). In the presence of this polymorphism, the STI domains of UBQLN1 are unable to bind to Presinilin proteins as a result of which a gamma secretase complex is formed generating $A \beta 40$ and $A \beta 42$ amyloid aggregations (Ford \& Monteiro, 2007; Mah et al., 2000; C. Zhang \& Saunders, 2009). Ford et al (2006) hypothesize that UBQ-8i polymorphism favors formation of UBQLN dimers and loses its ability to bind to PS proteins. Neurofibrillary tangles and Lewy bodies in brains of Alzheimer's disease and Parkinson's patients robustly stained for anti-UBQLN antibodies provide more evidence to the role of UBQLN in these diseases (Mah et al., 2000).

UBQLN1 is also implicated in pathogenesis of triplet repeat neurodegenerative diseases especially ones characterized by expanded polyglutamine (PolyQ) tracts. Of the nine different types of PolyQ diseases, UBQLN1 plays a role in Huntington's disease (HD) and spinocerebellar ataxia type1 (Riley \& Orr, 2006). The expansion of glutamine tracts leads to aggregation of the affected protein as it is unable to get degraded effectively in absence of UBQLN1. In an animal model of Huntington's disease, UBQLN4 expression was identified to be protective as it decreased polyQinduced protein aggregation though its interaction with Ataxin1 and UBQLN1 $(\mathrm{H}$. Wang \& Monteiro, 2007): UBQLN2 mutations have been identified in X-linked Amyotropic Lateral Sclerosis (ALS) and Fronto-Temporal Dementia (FTD). UBQLN2-positive 
inclusions are detected in spinal cord of ALS patients and in hippocampus of ALS patients with dementia proposing a role of mutant UBQLN2 aggregation in neurodegeneration (Deng et al., 2011).

\section{Ubiquilins in Cancer}

UBQLN1 is linked to development and progression of cancers such as neuroblastoma, lung adenocarcinomas, urothelial carcinoma and osteosarcomas. UBQLN1 aka DA41 in 1997, was identified as an interacting partner of a tumor suppressor protein DAN, frequently deleted in neuroblastoma (Ozaki et al., 1997). DA41 overexpression in Rous sarcoma virus transformed rat fibroblasts (Ras-3Y1), caused a decrease in cell proliferation and colony formation of these transformed cells (Ozaki, Nakamura, Hanaoka, Nakagawara, \& Sakiyama, 2000). Similarly, exogenous DAN expression in Ras-3Y1 cells depleted of endogenous DAN, caused arrest in their DNA synthesis. DA41 or UBQLN1 was believed to suppress overall growth through interaction with a cell cycle regulator, CDK2 and DAN. Additionally, DA41's STI domains (155-232) were mapped as region of interaction with another protein called $S(1-5)$, which bears several EGF like repeats, also capable of controlling cell cycle (Ozaki et al., 1997). Funakoshi and colleagues found XDRP1 in 1999, a Xenopus orthologue that is $60 \%$ similar to Ubiquilin1, which interacted with another cell cycle regulator, Cyclin A, preventing its degradation and arresting cell division in frog embryo. Basic helix-loop-helix (bHLH) proteins are a large family of transcription regulators that function in critical developmental processes of the nervous system. $\mathrm{HASH}-1$ is a bHLH protein, overexpressed in pediatric malignancies like neuroblastoma. HASH-1 and HES-1, another bHLH protein, interact with UBQLN1 in neuroblastoma cells. Overexpression of UBQLN1 leads to their accumulation and coexpression of HASH-1 and UBQLN1 causes UBQLN1 to translocate to the nucleus (Persson, Stockhausen, Pahlman, \& Axelson, 2004). This was the first evidence that UBQLN1 is involved in neurogenesis. Furthermore, 2DE proteome analysis of a 
human fetal midbrain stem cell line identified downregulation in expression of UBQLN1 as the stem cells underwent differentiation from Day 0 to Day 7 (Hoffrogge et al., 2006). Therefore, aberrations in UBQLN1 function can potentially lead to developmental irregularities and even loss of cell cycle control as in cancer. UBQLN1 mRNA and protein levels were increased in the nine tumor and adjacent normal tissues examined and UBQLN1 overexpression was identified as a target candidate for humoral immune response in sera of 2 large lung cancer patient samples (150 patient vs. 100 control and 62 patient vs. 60 control) (Chen et al., 2007). Shimada et al report that urothelial cancer cells produce high levels of reactive oxygen species and consequently have increased levels of UBQLN2 to protect from ROS induced damage. Therefore, immunocytochemistry staining of UBQLN2 can be used to distinguish between neoplastic and non-neoplastic cells in urine. Additionally, nuclear staining of urothelial cancer cells corresponded with a higher grade of cancer (Shimada et al., 2016). A cisplatin-sensitive human ovarian carcinoma cell line was treated with AuL12 and $\mathrm{Au}_{2}$ Phen, cytotoxic gold compounds, known to be potent antiproliferative agents to discover that these compunds resulted in increased expression of UBQLN1 which inhibited degradation of p53 and thus prevented tumor proliferation (Guidi et al., 2012). UBQLN1 was reported to be lost in approximately fifty percent of non-small cell lung adenocarcinomas (Shah et al., 2015). siRNA mediated loss of UBQLN1 and mir155 mediated downregulation of UBQLN1 in lung cancer cells has been shown to promote an EMT-like phenotype. It has been shown that downregulation of UBQLN1 leads to a significant increase in mesenchymal markers, including Vimentin, Snail and ZEB1 and therefore, UBQLN1 has been suggested to play a role in suppression of metastasis in lung cancer (Yadav et al., 2017). However, overexpression of UBQLN1 in lung cancer cells (Chen et al., 2007) and UBQLN2 in osteosarcomas (Tsukamoto et al., 2015) have also been reported. At the protein level, UBQLN1 stabilizes tumor suppressor p53 (Feng et al., 2004; Kleijnen et al., 2000) as well as anti-apoptotic protein BCLb (Beverly et al., 2012; Kurlawala et al., 2017). Previous work from our lab has identified UBQLN1 
as a regulator of anti-apoptotic protein Bcl2L10/Bclb (Beverly et al., 2012). We have reported that UBQLN1 is lost in about $50 \%$ of lung adenocarcinomas and tissue samples have varying UBQLN1 mRNA levels. We have also shown that loss of UBQLN1 or UBQLN2 promotes epithelial to mesenchymal transition (EMT) in lung adenocarcinoma cell lines (Shah et al., 2015). Thus, it is fallible to conclude a definitive role of UBQLN proteins in cancer development until large sample sizes and deeper stratification of patients and subtypes of cancers have been investigated. Equally important is understanding how Ubiquilin1 differentially regulates multiple substrates thus providing a clearer insight into Ubiquilin related diverse findings in cancers.

Considering the range of substrates and cellular processes Ubiquilin1 is involved in, we hypothesize that Ubiquilin1 is a versatile, multi-purpose adaptor, housing domains for binding to other proteins leading to functional specificity of resulting multimeric complexes. 


\section{Overall Hypothesis}

Fate of substrates that UBQLN1 associates with is interaction-domain specific.

\section{Overall Goal}

The overall goal of this dissertation work is to understand the mechanism of action of UBQLN1 in regulating BCLb and IGF1R.

\section{Specific Aims}

\section{To investigate biochemical functions of UBQLN1}

i. Identify domains of UBQLN1 responsible for interaction with multiple substrates

ii. Determine role of UBQLN1 on substrate stability

\section{To investigate role of UBQLN1 in regulation of receptor tyrosine kinases}

i. Determine role of UBQLN1 in trafficking and turnover of IGF1R

ii. Determine biological consequences of UBQLN1 loss and dysregulation of IGF pathway in lung adenocarcinoma cells.

iii. Determine role of UBQLN1 in regulation of INSR and EGFR

UBQLN1 either facilitates degradation or stabilizes substrates it binds to. The signal that determines this fate is unknown. Data supporting the existing working model of UBQLN1 is variable. Using BCLb as a model substrate, we examined characteristics of UBQLN1-BCLb and UBQLN1-IGF1R interaction. We hypothesize that UBQLN1 expression normally acts to prevent untimely loss of IGF1R and terminate phosphorylation signal of activated IGF1R in lung adenocarcinoma cells. By doing so, it aids in controlled regulation of downstream signaling pathways. We studied in detail how loss of UBQLN1 is involved in altering mechanisms of IGF1R expression and activity. 


\section{CHAPTER 2}

\section{MATERIALS AND METHODS}

\section{Cell Culture}

293T cells were cultured in DMEM supplemented with 10\% FBS. DNA transfections were done using PEI (2.5:1). All cell extracts were prepared following scrape harvesting of 293T cells using CHAPS lysis buffer (1\% CHAPS detergent, $150 \mathrm{mM} \mathrm{NaCl}, 50 \mathrm{mM}$ Tris $\mathrm{pH}$ 7, 5 mM EDTA). Subcellular fractionation experiments were performed using the ProteoExtract Subcellular Proteome Extraction kit (Calbiochem/Merck), as per the manufacturer's protocol. For immunoprecipitations, $400 \mathrm{ug}$ of protein was incubated in $400 \mathrm{uL}$ of total CHAPS buffer and incubated with indicated affinity matrix for $1 \mathrm{~h}$ at $4{ }^{\circ} \mathrm{C}$. Following incubation, the matrix was washed three times in CHAPS buffer and then SDS loading buffer was added directly to washed matrix, boiled, and loaded directly into the wells of a PAGE gel. Drug treatments were performed as described in the text using 20uM Cycloheximide, 25 uM proteasome inhibitor MG132, 360nM Epoximide, 1uM Bortezomib. Human non-small cell lung adenocarcinoma cell lines A549 were purchased from American Type Culture Collection (ATCC, Rockville, MD, USA) and cultured in RPMI medium supplemented with 10\% fetal bovine serum (Invitrogen, Carlsbad, CA, USA) and $1 \%$ antibiotic/antimycotic (Sigma, St Louis, MO, USA). The 3 cell lines were routinely subcultured every 3-4 days. All siRNA transfections were performed using Dharmafect1 \#T-2001-03 (Thermo Fisher Scientific Inc, Pittsburgh, PA, USA) as per manufacturer's protocol. After total 48 hours of transfection, cells were serum starved for 12 hours. After 
which IGF1 $50 \mathrm{ng} / \mathrm{ml}$ was added to stimulate the cells. For protein stability studies, Cycloheximide (20uM), and for testing degradation pathways, Bortezomib (1uM) and Monensin (10uM) were added an hour prior to stimulating with IGF1. At the end of stimulation for various time points, cells were harvested in CHAPS lysis buffer ( $1 \%$ CHAPS detergent, $150 \mathrm{mM} \mathrm{NaCl}, 50 \mathrm{mM}$ Tris $\mathrm{pH} 7,5 \mathrm{mM}$ EDTA) supplemented with protease and phosphatase inhibitors. Protein was quantified by using Pierce's BCA Protein Assay Reagent Kit (\# 23227) from Pierce Biotechnology, Rockford, IL, USA as per manufacturer's protocol.

\section{Plasmids}

All experiments using expression of BCLb proteins were performed with vectors engineered for this study. The following accession numbers were used to design oligos for PCR based cloning from cDNA libraries generated from a "universal human RNA" library (Stratagene/Agilent Technologies): BCLb \#NM_020396. FLAG-tagged BCL2 constructs were generated by performing PCR of the MIG- BCL constructs using an oligo that fused the FLAG coding sequences to the amino terminus of each BCL2-protein. This product was then subcloned back into the MIGRX vector. The following plasmids were obtained from Addgene; FLAG-Ubqln (Addgene plasmid 8663, deposited by Peter Howley), FLAG-Ubqln-112X (Addgene plasmid 8664, deposited by Peter Howley), pRK5HA-Ubiquitin-WT (Addgene plasmid 17608, deposited by Ted Dawson), pRK5-HAUbiquitin- K0 (Addgene plasmid 17603, deposited by Ted Dawson), pRK5-HA-UbiquitinK48 (Addgene plasmid 17605, deposited by Ted Dawson), GFP- BCL2 (Addgene plasmid 17999, deposited by Clark Distelhorst). The GFP-tagged BCLb construct was purchased from OriGene catalogue \#RG211604. Lysine-less BCLb construct was engineered using the MIG- or MIT-BCLb constructs described above to do site directed mutagenesis with the Quick Change Multi Site Mutagenesis kit (Stratagene/Ambion), as per the 
manufacturer's protocol. MYC-UBQLN1 construct was a gift from Annakaisa Haapasalo, Finland. Domain deletion constructs of UBQLN1 were created using the Q5 Site-Directed Mutagenesis Kit (New England Biolabs). Antibodies-BCLb \#ab45412 and GFP \#ab6673 (Abcam); VDAC \#4866 (Cell Signaling); IGF1R \#3018 (Cell Signaling), ESYT2 \#HPA002132 (SIGMA), S5a/PSMD4 \#12441 (Cell Signaling), BAG6 \#A302-039A-T (Bethyl), MYC \#F2512 (Santa cruz), Tubulin \#B512, FLAG M2 conjugated agarose beads, FLAG poly-clonal \#F7425 (Sigma); UBQLN poly-clonal was made by inoculating rabbits with a peptide specific to UBQLN1 (Yenzym Antibodies LLC); HA affinity matrix and HA \#3F10 (Roche).

\section{Immunoprecipitation and Western Blot Analysis}

HEK 293 T cells were cultured in DMEM supplemented with 10\% FBS. DNA transfections were done using PEI (PEI 2.5:1 DNA). All cell extracts were prepared following scrape harvesting of 293T cells using CHAPS lysis buffer (1\% CHAPS detergent, $150 \mathrm{mM} \mathrm{NaCl}$, $50 \mathrm{mM}$ Tris $\mathrm{pH} 7,5 \mathrm{mM}$ EDTA), For immunoprecipitation, 400ug of protein was incubated in 400uL of total CHAPS buffer and incubated with indicated affinity matrix (Anti-FLAG beads) for $1 \mathrm{~h}$ at $4{ }^{\circ} \mathrm{C}$. Following incubation, the matrix was washed three times in CHAPS buffer and then SDS loading buffer was added directly to washed matrix, boiled, and loaded directly into the wells of a PAGE gel.

\section{Immunofluorescence}

HEK 293T or HeLa cells were transfected with FLAG-UBQLN1, dry seeded on round coverslips in 12 well plates and 48 hours post transfection were fixed with $4.0 \%$ paraformaldehyde in PBS for $15-20$ min and then permeabilized with $0.1 \%$ Saponin for 60 minutes at room temperature. Cells were rinsed thrice with PBS, and then incubated overnight with anti-UBQLN1 antibody (CST\# 1:1000). Next day, after three successive 
washes with PBS, cells were then incubated with Alexa Fluor 488 goat anti-rabbit IgG (1:300, A11034 Molecular Probes, Invitrogen detection technologies, Eugene, OR, USA). After incubation with secondary antibody for 60 minutes, cells were rinsed with PBS and incubated with Alexa Fluor 568 Phalloidin (1:1000 A12380: Life technologies Eugene, OR, USA) for 10 minutes. After 3 successive washes with PBS, nuclei were counterstained with DAPI (1:000) for $10 \mathrm{~min}$ at room temperature followed by three washes $(5-10 \mathrm{~min}$ each) with PBS. The cells were then imaged under Nikon A1R confocal laser scanning microscope. Multiple images were acquired from multiple experiments and representative images are presented.

\section{Radioligand Binding Assay}

Cells were incubated for 2 hours with increasing concentrations $(0.001 \mathrm{nM}$ to $0.05 \mathrm{nM})$ of ${ }^{125}$ I-IGF1 (Perkin Elmer, Waltham, MA) at $4^{\circ} \mathrm{C}$ in binding buffer (DMEM/20 mM HEPES/0.1\% BSA, pH 7.3). Two fractions were collected - total ligand binding ( ${ }^{125}$ I-IGF1) and non-specific binding (mixture of radiolabeled and non-radiolabeled ligand). After 2 hours incubation on ice, cells were washed four times with binding buffer to remove unbound radioligand. Solubilizing buffer $(0.5 \mathrm{~N}$ SDS, $1 \mathrm{~N} \mathrm{NaCl})$ was used to collect the cells and the radioactivity of each fraction was determined using a Beckman Coulter (Brea, CA) gamma counter. Specific binding for each concentration of ${ }^{125}$ I-IGF1 was calculated by subtracting non-specific binding from total binding for that concentration of ${ }^{125}$ I-IGF1. Bmax and Kd (cpm) were calculated by PRISM analysis based on Specific Binding values (non specific subtracted by total binding). Bmax ( $\mathrm{mmol}), \mathrm{Kd}(\mathrm{nmol} / \mathrm{L})$ and number of receptors were calculated as in picture. 
* Specific activity of $\mathrm{I}^{125}$-IGF $1=3.829 \times 10^{15} \mathrm{dpm} / \mathrm{mmol}$

* $\mathrm{B}_{\max }=16542 \mathrm{cpm}$ (PRISM analysis) (one-site binding)

* Correction for machine $=0.45$

* $\mathrm{Kd}=33,095 \mathrm{cpm}$ (PRISM analysis)

1. $\mathrm{B}_{\max }($ in $\mathrm{mmol})=16,542 \mathrm{cpm} \times \frac{1 \mathrm{dpm}}{0.45 \mathrm{cpm}} \times \frac{1 \mathrm{mmol}}{3.229 \times 10^{15} \mathrm{dpm}}$

$$
=9600.417 \times 10^{-15} \mathrm{mmol}
$$

2. Receptors/assay $=9600.417 \times 10^{-15} \mathrm{mmol} \times \underline{6.023 \times 10^{23}}$ mole

$$
=57.8 \times 10^{5} \text { receptors/assay }
$$

3. Receptors $/$ cell $=\underline{57.8 \times 10^{5}}=\mathbf{4 6 . 9 9}$

4. $\mathrm{Kd}($ in $\mathrm{nmol})=33,095 \mathrm{cpm} \times 1 \mathrm{dpm} \times 1 \mathrm{mmol}$

$\overline{0.45 \mathrm{cpm}} \overline{3.829 \times 10^{5} \mathrm{dpm}}$

$$
=1.9 \times 10^{-5} \mathrm{nmol}
$$

5. Reaction $\mathrm{Vol}=250 \mu \mathrm{L}$

$\therefore \quad \mathrm{Kd}(\mathrm{nmol} / \mathrm{L})=\frac{1.9 \times 10^{-5} \mathrm{nmol}}{0.250 \times 10^{-3} \mathrm{~L}}=0.076 \mathrm{nmol} / \mathrm{L}$

Example of calculations for Bmax, $\mathrm{Kd}$, receptor numbers 


\section{Percoll Gradient Density Centrifugation}

A549 cells expressing UBQLN1 shRNA (shUBQLN1 and shControl) $\left(15 \times 10^{6}\right.$ cells) were seeded onto $15 \mathrm{~cm}^{2}$ plate and cultured for 12 hours in serum-free conditions before stimulating with IGF1 for 1 hour followed by harvesting, lysing and loading onto a Percoll gradient to fractionate organelles makers based on relative density. Detailed methods are described by Kornilova and Sorkin (Kornilova, Sorkina, Beguinot, \& Sorkin, 1996). These experiments were performed 3 separate times and data here are representation of a single experiment.

\section{Survival Assay}

A549 cells expressing UBQLN1 shRNA (U1KD\#1, U1KD\#2 and control) $\left(2 \times 10^{4}\right.$ cells) were seeded onto 96 well plate and cultured for 3 days under 3 different conditions-serum-free media, serum-free media supplemented with IGF1 $(50 \mathrm{ng} / \mathrm{ml})$ or serum-free media supplemented with Linsitinib (1uM). Cell viability was assessed by Alamar Blue assays on each day. Data were normalized to control shRNA cells in serum-free condition. These experiments were performed 2 separate times and data here are representation of a single experiment.

\section{Transwell Migration}

A549 cells expressing UBQLN1 shRNA (shUBQLN1, shUBQLN2 and control) $\left(2.5 \times 10^{4}\right.$ cells $)$ were seeded onto the wells of Transwell filters in a 12-well plate, according to the manufacturer's instructions (Corning). The Transwell setup consisted of an upper chamber (conditions of serum-free media, serum-free media supplemented with IGF1 $(50 \mathrm{ng} / \mathrm{ml})$ or serum-free media supplemented with Linsitinib (1uM)) that was placed onto a lower chamber (RPMI media enhanced with 10\% FBS, creating a chemotactic gradient). The upper chamber contained a microporous membrane allowing passage of 
cells that migrate towards serum. After incubation at $37 \mathrm{C}$ and $5 \% \mathrm{CO}_{2}$ for 24 hours, the inserts were examined for migration in the above 3 conditions. Results were compiled as the mean and standard error of 3 separate experiments.

\section{Total RNA Extraction and Real Time PCR}

Total RNA was isolated from the A549 cells after washing twice with phosphate-buffered saline (PBS) and harvested with E.Z.N.A Total RNA Extraction Kit (Omega, USA) according to the supplier's protocol followed by DNAse digestion. RNA quality and quantity were determined by photometry. Total RNA $(1 \mu \mathrm{g})$ was reverse-transcribed to cDNA using Thermo Script RT-PCR kit. Briefly, RNA was reverse-transcribed in cDNA with oligo (dT) primers and $200 \mathrm{U}$ of Superscript II (Invitrogen) following manufacturer's instructions. Real-time analysis for IGF1R, INSR, IGF1, IGF2, Insulin and normalizing gene human $\beta 2$ Microglobulin or $\beta$-Actin was performed using SYBR Green Master Mix as per the manufacturer's instruction (Applied Biosytems). This technique continuously monitors the cycle-by-cycle accumulation of fluorescently labeled PCR product. Briefly, cDNA corresponding to $25 \mathrm{ng}$ of RNA served as a template in a $10 \mu \mathrm{l}$ reaction mixture containing, $0.2 \mathrm{nM}$ (each) primer, and 5 $\mu \mathrm{l}$ FastStart DNA Master SYBR Green mix (ABI). Samples were loaded into 96-well plate format and incubated in the fluorescence thermocycler 7500 (ABI System). Initial denaturation at $95^{\circ} \mathrm{C}$ for 10 min was followed by 45 cycles, each cycle consisting of $95{ }^{\circ} \mathrm{C}$ for $15 \mathrm{~s}$, touchdown of $1{ }^{\circ} \mathrm{C} /$ cycle from the primer-specific start to end annealing temperatures for 5 seconds, and $60{ }^{\circ} \mathrm{C}$ for 10 seconds. The primer sequences used for specific genes are listed in Table. All quantifications were normalized to the housekeeping HPRT gene, which showed a very stable expression in A549 cells. Fold changes in gene expression were calculated using $2^{-\Delta \Delta C T}$ method. Following are the primer sequences used for the reaction:

1. IGF1R: 
F: GCCAAGCTAAACCGGCTAA

R: TATCCTCTTTTGGCCTGGACATA

2. IGF1

F: GATGCACACCATGTCCTCCT

R: AAAAGCCCCTGTCTCCACAC

3. IGF2:

F: AGTTCTTCCAATATGACACCTGGAA

R: TGAACGCCTCGAGCTCCTT

4. B2M

F: TGACTTTGTCACAGCCCAAGATA

R: AATGCGGCATCTTCAAACCT

5: ACTIN

F: TTGGCAATGAGCGGTTCC

R: GGTAGTTTCGTGGATGCCCAC

\section{Antibodies}

IGF1R-beta (CST\#3027), IGF1R Beta XP (CST\#9750), p-IGF1R beta (CST \#3918), AKT (CST \#9272), p-AKT (CST\#9271); Tubulin \#B512 (Sigma); GAPDH \#FL335 (Santa Cruz); Actin (Sigma\#A5316), Ubiquilin1 (CST\#14526); Anti-FLAG Affinity Gel (Sigma A2220), INSR (CST \#3025), FLAG, EEA1, TfnR

\section{RNAi Sequences}

All RNAi (siRNAs) used for study were ordered from Thermo Fisher Scientific Biosciences Inc. Lafayette, CO 80026, USA and transfections were done using Dharmafect1 as per the supplier's instructions.

1. Non-Targeting Control UAAGGCUAUGAAGAGAUACAA

2. UBQLN1

\#1: GAAGAAAUCUCUAAACGUUUUUU

\#2: GUACUACUGCGCCAAAUUU 


\section{CHAPTER 3}

\section{INTRODUCTION}

The Ubiquilin (UBQLN) family of proteins (UBQLN1-4, UBQLN-L) is evolutionarily conserved and structurally similar. UBQLN1 is $\sim 63 \mathrm{kDa}$ protein and all its family members have 3 functional domains: ubiquitin- like domain (UBL) at the $\mathrm{N}$ terminus, ubiquitin- associated domain (UBA) at the C-terminus and STI chaperonelike regions in the middle (Fig. $1 A$ ). UBQLN1 is ubiquitously expressed in all tissues. Mutations and loss of the UBQLN1 gene have been detected in a variety of disorders ranging from neurodegenerative disorders like Alzheimer's disease (1) to cancers like non-small cell lung adenocarcinoma (2). Initially, UBQLN1 was discovered as a protein that interacted with IAP (CD47) in ovarian cancer cells, and upon loss, increased cell spreading (3). However, since then UBQLN1 has been largely studied in the context of neurodegenerative disorders and protein quality control. UBQLN1 assists in proteasomal degradation of ubiquitinated substrates and also participates in ERAD (Endoplasmic Reticulum Associated Degradation) (2), autophagy (4,5), apoptosis (6) and receptor trafficking (7). As a UBL-UBA protein, UBQLN1 is hypothesized to bind to poly-ubiquitin chains of substrate through its UBA domain and shuttle it to the $19 \mathrm{~s}$ proteasome via its UBL-S5a cap interaction. The central region of UBQLN1 consists of STI domains that are called so because of their similarity to STI-1 proteins (STress Inducible proteins or Hsp70-Hsp90 organizing protein). This region acts as a cochaperone and mediates hydrophobic interactions with other proteins $(8,9)$. There is conflicting data to support the existing working model of UBQLN1. Several stu 
have reported that UBQLN1 facilitates proteolysis of substrates that it binds. Dsk2p, the yeast homolog of UBQLN1, forms a trimeric complex with Rad23 and Cdc48 and enables degradation of misfolded proteins via the proteasomal system (10). In human cells, the UBL domain of UBQLN1 has been shown to regulate degradation of misfolded proteins like Ataxin3, a deubiquitinase enzyme, HSJ1a, a protein that stimulates activity of Hsp70, and EPS15, a protein important in endosomal trafficking (11). UBQLN1 expression aids in poly-ubiquitination of viral polymerase NS5B, decreasing its half-life in HCV infected hepatocytes (12). In contrast, UBQLN1 stabilizes proteins like Presenilin1/2 $(13,14)$, IkBa (15), and BCLb (16) when bound to them. These data indicate that UBQLN1 either stabilizes or facilitates degradation of substrates that it binds to. The signal that determines this fate is unknown.

In this study, we aimed to understand characteristics of substrates that bind to UBQLN1 and whether the nature of UBQLN1-substrate interaction decides the fate of substrates - stabilization or degradation. We show that Ubiquilin interacts with multiple substrates through its STI or UBL domains. We also show that UBQLN1 is capable of forming dimers and continues to interact with proteins in its dimerized form. We have previously published that UBQLN1 interacts with the anti-apoptotic BCL2 protein BCLb and stabilizes it in the cytoplasm. Here, we have used BCLb as a model to characterize the nature of its interaction with UBQLN1. 


\section{RESULTS}

\section{Cellular distribution of UBQLN1}

HPL1D, non-transformed lung epithelial cells and HeLa cells transfected with FLAGtagged UBQLN1 were examined for UBQLN1 staining by indirect immunofluorescence. Both endogenous (green in HPL1D cells) and exogenous UBQLN1 (red in HeLa cells) are ubiquitously expressed in the cytosol (Fig. 2A). However, in both these conditions, UBQLN1 is distinctly absent from nuclei and vacuolar compartments. To further examine if UBQLN1 associated with specific compartments of cells, we collected supra-nuclear lysates from Hela cells and loaded onto Percoll for density gradient centrifugation of proteins (Fig. 2B). There was slightly higher detection of UBQLN1 towards lower density gradients but overall, UBQLN1 seems to be expressed throughout the cytoplasm and possibly associates with multiple cellular compartments and organelles. Na-K-ATPase, an enzyme found in cell membrane of all animal cells is used as a control protein for lower density gradient fractions. 


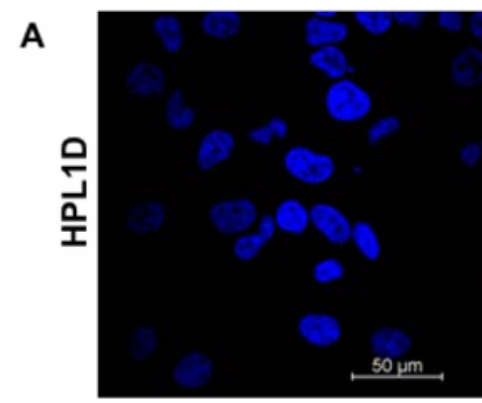

DAPI

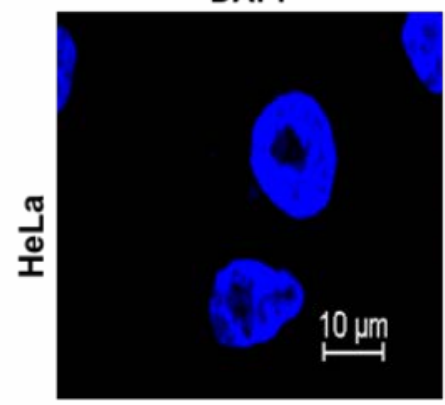

DAPI

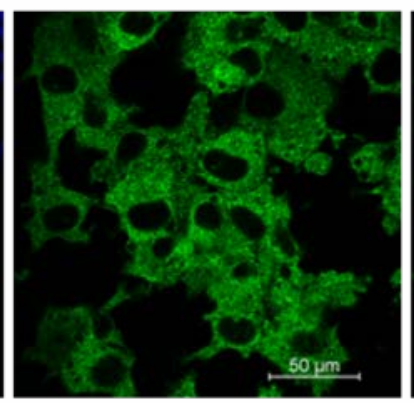

UBQLN1

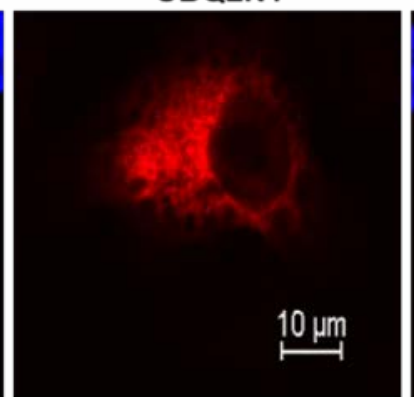

FLAG-UBQLN1

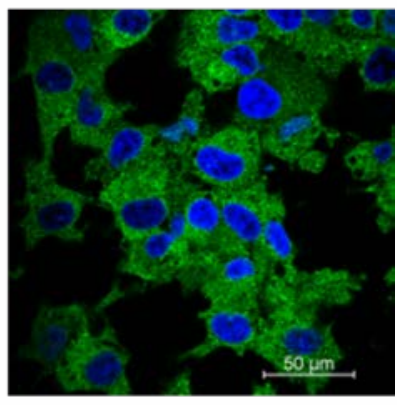

MERGE

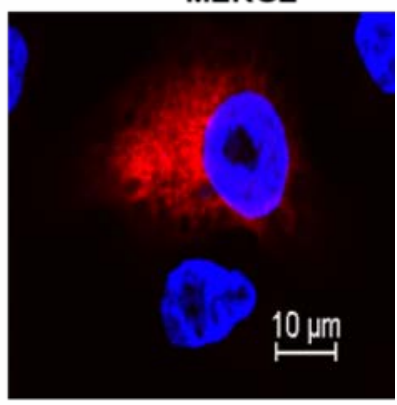

MERGE

B (High Density)

(Low Density)

Rf 1.0 0

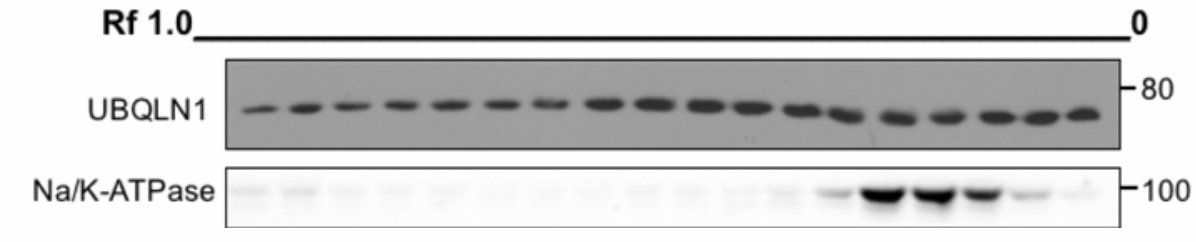

Figure 2: Cellular Distribution of UBQLN1

A: Confocal microscopy images of indirect immunofluorescence staining of endogenous UBQLN1 (green) in normal lung epithelial HPL1D cells and over-expressed FLAG-tagged UBQLN1 (red) in HeLa cells. Both endogenous and exogenous UBQLN1 are ubiquitously expressed in the cytosol. However, in both these conditions, UBQLN1 is distinctly absent from nuclei and vacuolar compartments.

B: Supra-nuclear lysates from Hela cells were loaded onto Percoll for density gradient centrifugation of proteins. Overall, UBQLN1 was detected in all fractions with slightly higher expression towards the lower density gradients. $\mathrm{Na} / \mathrm{K}-\mathrm{ATPase}$, an enzyme found in cell membrane of all animal cells is used as a control protein for lower density gradient fractions. Density fractions were prepared by Julie A. Gosney. (Data are from one experiment). 
UBQLN1 non-selectively interacts with diverse ubiquitin linkages through its UBA domain

Our previous work suggested that UBQLN1 interacts with mono-ubiquitinated BCLb (16). However, previous work by Feng et al (15) and Zhang et al (17) showed that UBA domain of UBQLN1 is also capable of associating with poly- ubiquitin chains. Therefore, we investigated if UBQLN1 interacts with specific ubiquitination linkages on substrates. We engineered constructs of UBQLN1 missing individual domains (Fig. $3 A$ ). Cells were transfected with empty vector (EV), FLAG UBQLN1 ${ }^{\mathrm{WT}}$ or deletion constructs of UBQLN1 (Fig. 3A) and HA-Ub ${ }^{\mathrm{WT}}$ (Ubiquitin) (Fig. 3B,C). FLAG-UBQLN1 was immunoprecipitated and interaction with HA-Ubiquitin was examined. We confirmed that the UBA domain of UBQLN1 interacts with ubiquitin as constructs missing the UBA domain, namely UBQLN1 $1^{112 X}$ and UBQLN1 $1^{\triangle \mathrm{UBA}}$ fail to pull down HAUbiquitin. Next, we performed a similar experiment using our individual domain deletion constructs (Fig. 3A) and confirmed that $\triangle U B A$ does not interact with ubiquitin. Interestingly, $\Delta \mathrm{STI}-4$ construct also failed to pull down ubiquitin probably because of STI-4 domain's close proximity to the UBA domain such that when STI-4 is deleted, it directly impacts UBA domain's ability to interact with ubiquitin. Next, we tested for preference of the UBA domain of UBQLN1 for diverse ubiquitin linkages. We used constructs of HA-Ubiqutin ${ }^{W T}$ that have all Lysines $(K)$ mutated to Arginine $(R)$ except one, for example, HA-UBQTN ${ }^{\mathrm{K} 33}$ has all K's mutated to R except $\mathrm{K} 33$ and therefore overexpression promotes formation of $\mathrm{K} 33$ ubiquitin chains on substrates. $\mathrm{K} 0$ indicates all K's have been mutated to $R$ and this ubiquitin molecule can be conjugated to substrate (mono- ubiquitination), but is not capable of forming ubiquitin chains. Results show that UBQLN1 is capable of interacting with WT, K33-, K48-, K63- poly-ubiquitin chains and mono-ubiquitinated residues (Fig. 3D). Based on these data and literature (17) we conclude that UBQLN1 non-selectively interacts with diverse ubiquitin linkages on substrates via its UBA domain. 

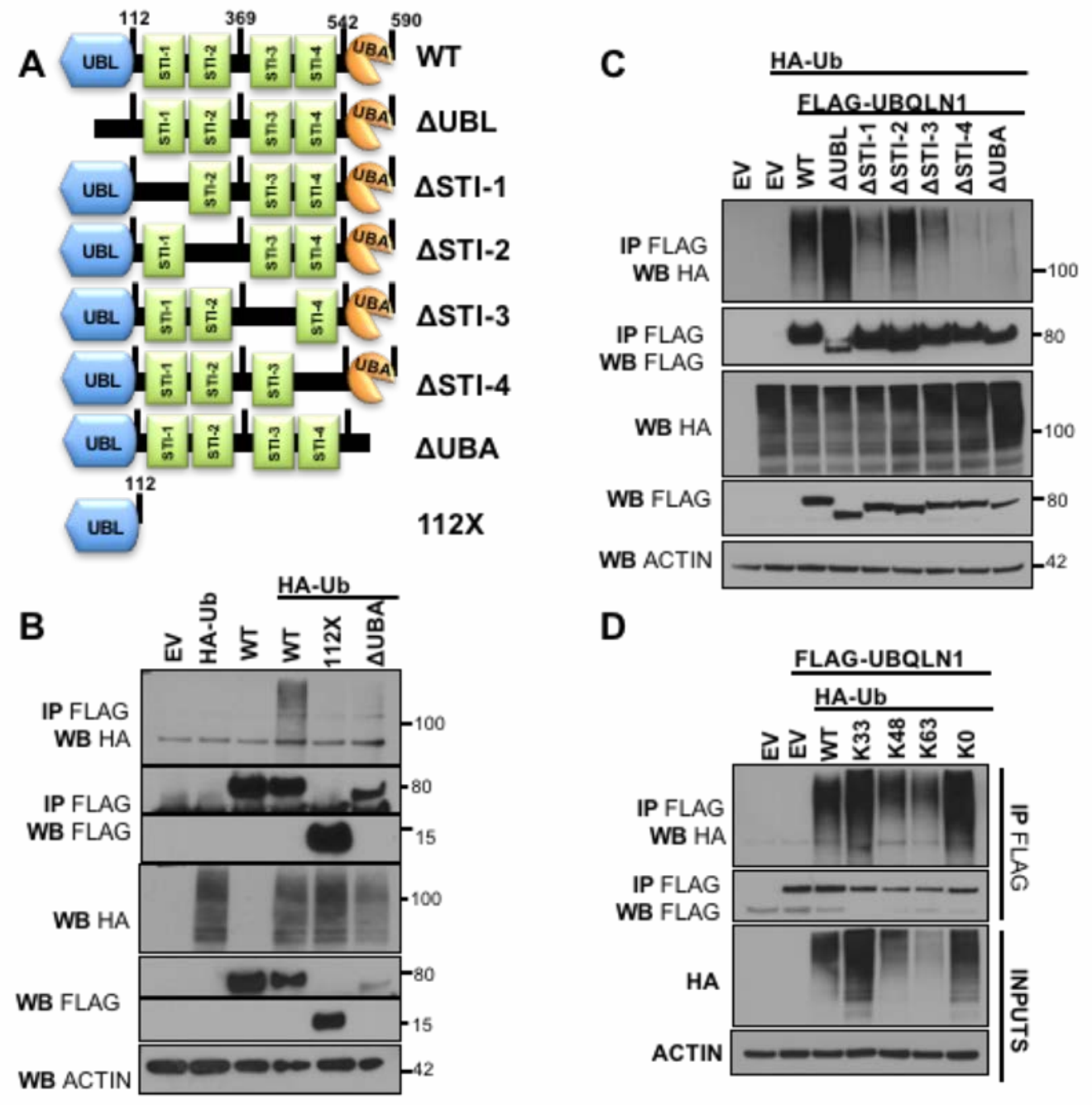

Figure 3: UBQLN1 interacts non-preferentially with diverse ubiquitin linkages through its UBA domain.

(A) Schematic of UBQLN1 ${ }^{\mathrm{WT}}$ and engineered domain deletion constructs missing individual domains. (B,C), 293T cells were transfected with empty vector $(E V)$, FLAG-UBQLN1 ${ }^{\text {WT }}$, domain deletion constructs of UBQLN1 and HA-Ubiquitin ${ }^{\text {WT }} .48$ hours post transfection, cells were lysed and Western Blot analyses were performed with the indicated antibody. Where indicated, immunoprecipitation with anti-FLAG conjugated agarose beads was performed. UBQLN $1{ }^{\mathrm{WT}}$ interacts with ubiquitin but UBQLN1 $1^{112 X}$ and UBQLN1 $1^{\triangle \mathrm{UBA}}$ (both missing UBA domain) lose this interaction. Therefore, UBQLN1 interacts with ubiquitin through the UBA domain. (D) 293T cells were transfected with FLAG-UBQLN1 ${ }^{\text {WT }}$ and constructs of HA-Ubiquitin ${ }^{\text {WT }}$ that have all Lysines (K) mutated to Arginine (R) except one for example, HA-Ubiquitin ${ }^{\mathrm{K} 33}$ has all K's are mutated to R except K33 and therefore overexpressing and promoting formation of K33 ubiquitin chains on substrates. K0 indicates all K's have been mutated to $\mathrm{R}$ and this ubiquitin molecule is can only conjugate to the substrate (mono-ubiquitination) and is incapable for forming ubiquitin linkages. UBQLN1 interacts with WT, K33-, K48-, K63- poly-ubiquitin chains and K0 mono-ubiquitinated substrates non-selectively. (Data are from one experiment). 


\section{Recognition of the transmembrane domain of BCLb by STI-1/2 domains of UBQLN1 sequesters BCLb in the cytosol}

Recently, Itakura et al (18) found that the UBQLN4 recognizes the transmembrane domain of mitochondrial protein OMP25. We have previously shown that UBQLN1 interacts with mitochondrial protein BCLb and stabilizes it in the cytosol. Here, we investigated the domain of UBQLN1 required for interaction with BCLb our deletion constructs of UBQLN1. We identified STI-1 and STI-2 domains on UBQLN1 as critical for interacting with BCLb. 293T cells were transfected with empty vector (EV), FLAGUBQLN1 ${ }^{\mathrm{WT}}$, FLAG-UBQLN1 domain deletion constructs, MYC-BCLb ${ }^{\mathrm{WT}}$ or MYC$\mathrm{BCLb}^{\triangle \mathrm{TM}}$. Where indicated, immunoprecipitation with anti-FLAG beads was performed and probed for MYC-BCLb by Western Blot analyses. STI-1 and STI-2 domains of UBQLN1 are required for interaction with BCLb (Fig. 4A). UBQLN1 ${ }^{\mathrm{WT}}$ and all domain deletion constructs except $\triangle U B A$, interact with multi mono-ubiquitinated BCLb, indicated by the ladder-like pattern at higher molecular weights. Thus, the UBA domain of UBQLN1 is obligatory to interact with ubiquitin on BCLb. Next, we determined that TM domain is essential for UBQLN1 to recognize and interact with BCLb. These data suggest a potential 2-fold interaction between UBQLN1 and BCLb such that that the STI domains of UBQLN1 associate with the TM domain of BCLb and the UBA domain associates with the ubiquitin on BCLb (Fig. 4B). Previously, we have shown that UBQLN1 interacts with BCLb ${ }^{\mathrm{WT}}$ and sequesters it in the cytosol. As we determined that UBQLN1 needs an intact TM domain of BCLb to interact with it, we tested if UBQLN1 alters the location of the $B C L b^{\triangle T M}$. We performed subcellular fractionation by differential centrifugation to determine changes in cellular location of $B C L b^{\triangle T M}$. Results show that UBQLN1 does not recognize $B C L b^{\triangle T M}$, and therefore, does not interact with it and has no effect on its cellular location (Fig. 4C). Our data confirm results by Itakura et al (18) that UBQLN proteins are capable of recognizing transmembrane domains of some proteins, like OMP25 and BCLb. 

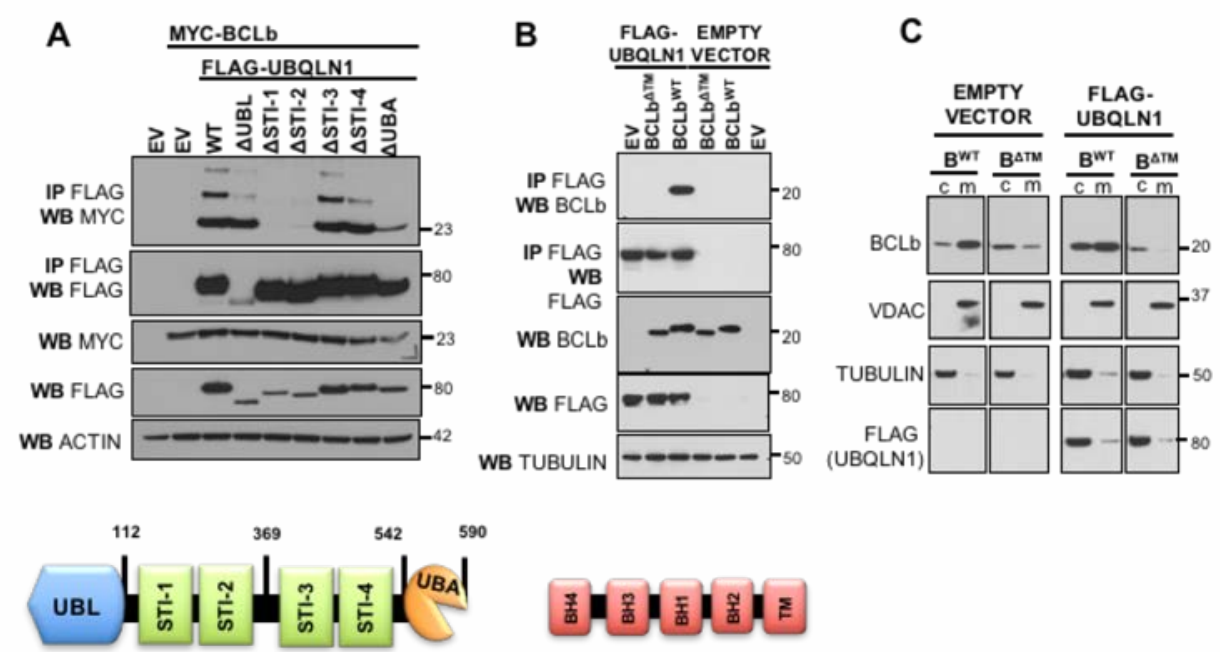

Figure 4: Recognition of the transmembrane domain of BCLb by STI$1 / 2$ domains of UBQLN1 sequesters $B C L b$ in the cytosol

The transmembrane domain of BCLb is recognized by STI-1/2 domains of UBQLN1 and stabilizes BCLb in the cytoplasm. 293T cells were transfected with empty vector (EV), FLAG-UBQLN $1^{\mathrm{WT}}$, or FLAG-tagged domain deletion constructs, MYC-BCLb ${ }^{\mathrm{WT}}$ or MYC-BCLb ${ }^{\triangle T M}$. Forty-eight hours post transfection, cells were lysed and Western Blot analyses were performed with the indicated antibody. Where indicated, immunoprecipitation with antiFLAG conjugated agarose beads was performed to determine interaction between UBQLN1 and BCLb proteins. (A) STI-1/2 domains of UBQLN1 are required to interact with $B C L b$. (B) BCLb ${ }^{\triangle T M}$ does not interact with UBQLN1. A functional transmembrane domain on BCLb is required for UBQLN1 to recognize and interact with it. (C) $293 \mathrm{~T}$ cells expressing $\mathrm{BCLb}^{\mathrm{WT}}$ or $\mathrm{BCLb}^{\Delta \mathrm{TM}}$ with EV or FLAG-UBQLN1, were subjected to subcellular fractionation, followed by Western Blot analyses. Cells were fractionated into cytosolic (c) and membranes (m). VDAC1, a membrane bound protein, and Tubulin, a cytoplasmic protein were used to document the validity of the fractionations. UBQLN1 does not interact with $B C L b^{\triangle T M}$ and therefore, does not alter the location of $B C L b^{\triangle T M}$, as it is unable to recognize it. (Data are from one experiment). 


\section{Interaction of UBQLN1 with BCLb is independent of ubiquitination of BCLb}

We showed above that the UBA domain which interacts with ubiquitin is dispensable for interaction of UBQLN1 with BCLb. BCLb has 4 lysines that can be potentially ubiquitinated (16) and therefore, we investigated whether ubiquitination of BCLb played a role in its interaction with UBQLN1. We generated a construct of BCLb $\left(B C L b^{\mathrm{K} 0}\right)$ in which all 4 lysines were mutated to arginine. We tested whether $\mathrm{BCLb}^{\mathrm{K} 0}$ is capable of being ubiquitinated and whether UBQLN1 interacts with non-ubiquitinated BCLb. Cells were transfected with $B C L b^{W T}$, lysine-less BCLb $\left(B C L b^{K 0}\right)$, HAUbiquitin $^{\mathrm{WT}}$, lysine-less Ubiqutin $\left(\mathrm{HA}-\mathrm{Ubiquitin}^{\mathrm{K} 0}\right.$ ) and FLAG- UBQLN1. Where indicated, immunoprecipitation with either anti-FLAG or anti-HA conjugated beads was performed to determine UBQLN1 - or Ubiquitin-interacting complexes, respectively as previously shown. BCLb ${ }^{\mathrm{WT}}$ gets mono- ubiquitinated on several of its lysine residues as observed by the ladder-like pattern of ubiquitination in $B C L b^{W T}$ in the presence of Ubiquitin $^{\mathrm{WT}}$. BCLb ${ }^{\mathrm{K} 0}$ does not get ubiquitinated. Non-ubiquitinated $\mathrm{BCLb} \mathrm{K}^{\mathrm{K} 0}$ also fails to be pulled down with anti HA-Ubiquitin antibody. Immunoprecipitating with anti-FLAG beads showed that UBQLN1 interacts with both $B C L b^{W T}$ and $B C L b^{K 0}$ implying that UBQLN1 interacts with BCLb in its ubiquitinated $\left(B C L b^{W T}\right)$ and non- ubiquitinated $\left(B C L b^{K 0}\right)$ forms. These data indicate interaction of UBQLN1 with BCLb is independent of ubiquitination of BCLb. 
A

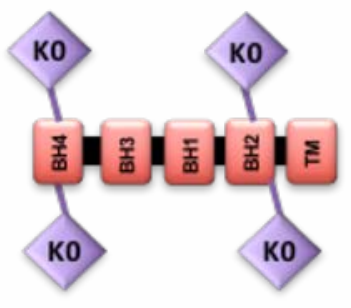

C

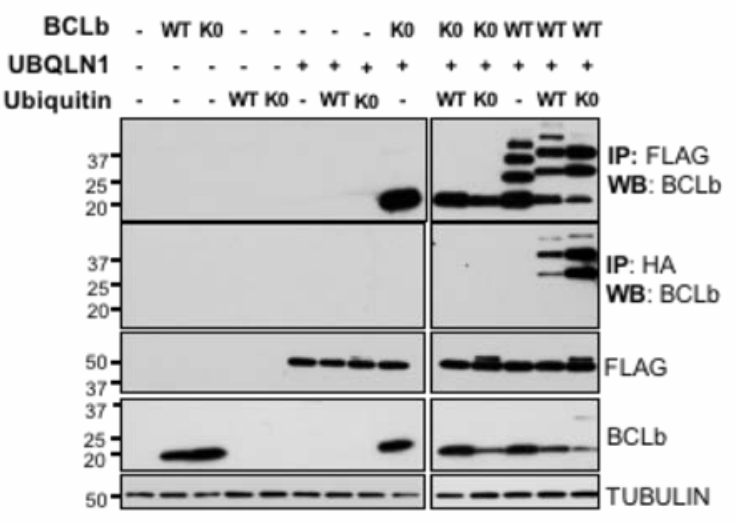

B

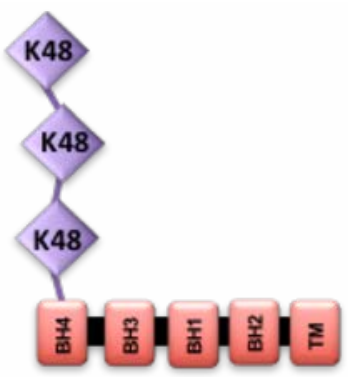

D

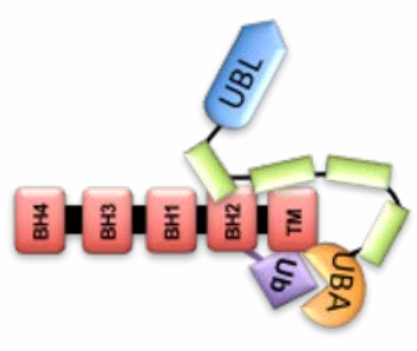

Figure 5: Interaction of UBQLN1 with BCLb is independent of ubiquitination of BCLb.

(A) Schematic of mono-ubiquitination on BCLb. Lysine residues are not mapped, picture is for representation purposes only. (B) Schematic of poly-ubiquitination of BCLb. Lysine residues are not mapped, picture if for representation purposes only. (C) 293T cells were transfected with BCLb (MIG-BCLb ${ }^{\text {WT }}$ ), lysine-less BCLb (MIG$\left.B C L b^{K 0}\right)$, wild-type Ubiquitin ( $\mathrm{HA}-\mathrm{Ub}^{\mathrm{WT}}$ ), lysine-less Ubiquitin (HA$\mathrm{Ub}^{\mathrm{K} 0}$ ), and FLAG-UBQLN1 ${ }^{\mathrm{WT}}$. Forty-eight hours post transfection, cells were lysed and Western Blot analyses were performed with the indicated antibody. Where indicated, immunoprecipitation with either anti-FLAG or anti-HA conjugated agarose beads was performed to determine FLAG-UBQLN1 or HA-Ubiquitin interacting complexes, respectively. $B C L b$ gets ubiquitinated on its lysine residues as seen by failure to pull down $B C L b^{K 0}$ by $\mathrm{HA}$-Ubiquitin. UBQLN1 interacts with both $\mathrm{BCLb}{ }^{\mathrm{WT}}$ and $\mathrm{BCLb}{ }^{\mathrm{K} 0}$ implying that UBQLN1 interacts with ubiquitinated (WT) and non-ubiquitinated (KO) BCLb as observed by the ladder-like pattern of ubiquitination in BCLb ${ }^{W T}$ in the presence of HA-Ubiquitin. Note; HA-tag adds $\sim 1 \mathrm{kDa}$ to the molecular weight of Ubiquitin. (Data are from one experiment). (D) Schematic showing UBQLN1 primarily interacts with BCLb through its STI domains and there may exist a secondary association between UBQLN1's UBA domain and ubiquitin conjugated on BCLb 


\section{Ubiquilin1 stabilizes $\mathrm{BCLb}^{\mathrm{WT}}$ after translational and proteasomal inhibitionWe}

We have previously shown (16) that BCLb gets completely degraded within 16 hours after blocking new protein synthesis by $\mathrm{CH}$. However, in the presence of UBQLN1 ${ }^{\mathrm{WT}}$, it is stabilized and protected from degradation. We wanted to determine whether ubiquitination of BCLb plays a role in its stability. Cells were transfected with BCLb ${ }^{\text {WT }}$ or $\mathrm{BCLb}$ engineered to lack all its four lysine residues $\left(B C L b^{\mathrm{K} 0}\right)$ or empty vector $(E V)$. Cells were treated with the indicated translational $(\mathrm{CH})$ and proteasomal (MG132, Epoximide, Bortezomib) inhibitors for 16 hours. GFP is used as an expression control since GFP is expressed from the same mRNA as BCLb following an internal ribosomal entry sequence. (Fig.7A). BCLb ${ }^{\mathrm{WT}}$ was completely degraded in the presence of $\mathrm{CH}$ for 16 hours while $\mathrm{BCLb}^{\mathrm{K} 0}$ expression was completely stable. This may indicate that $\mathrm{CH}$ induced degradation of $B C L b^{W T}$ is dependent on its lysines and therefore its ubiquitination, whereas degradation induced by proteasomal inhibition is independent of ubiquitination. UBQLN1 was co- transfected with $B C L b^{\mathrm{WT}}$ or $\mathrm{BCLb} \mathrm{K}^{\mathrm{K} 0}$ to test its effect on BCLb stability (Fig. 7B). Presence of UBQLN1 stabilized expression of BCLb ${ }^{\text {WT }}$ following both translational inhibition and proteasomal inhibition. Thus, UBQLN1 generally stabilizes $B C L b^{W T}$. Furthermore, UBQLN1 also stabilizes $B C L b^{\mathrm{K} 0}$ from proteasome inhibition- induced degradation. Immunoprecipitating with FLAG antibody demonstrated that UBQLN1 interacted with both $B C L b^{W T}$ and $B C L b^{K 0}$ under conditions of proteasomal inhibition by MG132 or translational inhibition by $\mathrm{CH}$, implying that primary interaction with BCLb is not lysine mediated (Fig. 7B). We then tested the effect of deletion constructs of UBQLN1 on stability of BCLb ${ }^{\text {WT }}$ (Fig.7C). UBQLN1 ${ }^{\triangle U B A}$ interacts with $\mathrm{BCLb}$, but fails to prevent loss of $\mathrm{BCLb}^{\mathrm{WT}}$ when exposed to $\mathrm{CH}$. We conclude that interaction of UBQLN1 with ubiquitin is dispensable for interaction with BCLb, but secondary interaction with ubiquitin on substrates is essential for its stabilization. As the transmembrane domain of BCLb is required for interaction with UBQLN1, we tested its stability under similar conditions and found that by itself 
$B C L b^{\triangle T M}$ is less stable than BCLb ${ }^{W T}$. However, an interesting observation is that BCLb lacking the transmembrane domain is the only construct that is stabilized following inhibition of the proteasome by MG132. Further, presence of UBQLN1 doesn't not alter stability of BCLb ${ }^{\Delta T M}$ (Fig. 7D), as it is unable to interact with it. Therefore, interaction between UBQLN1 and BCLb is independent of ubiquitination of BCLb but is required for stabilization of BCLb. 

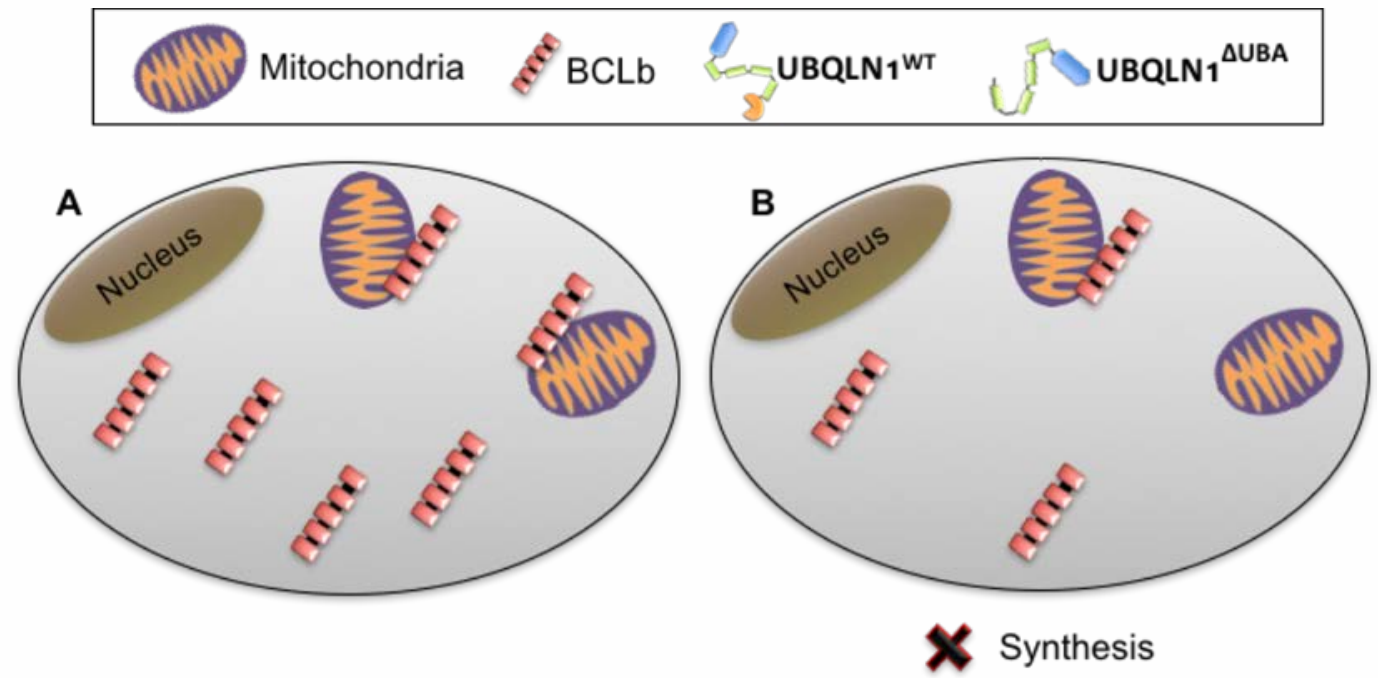

X Proteasome
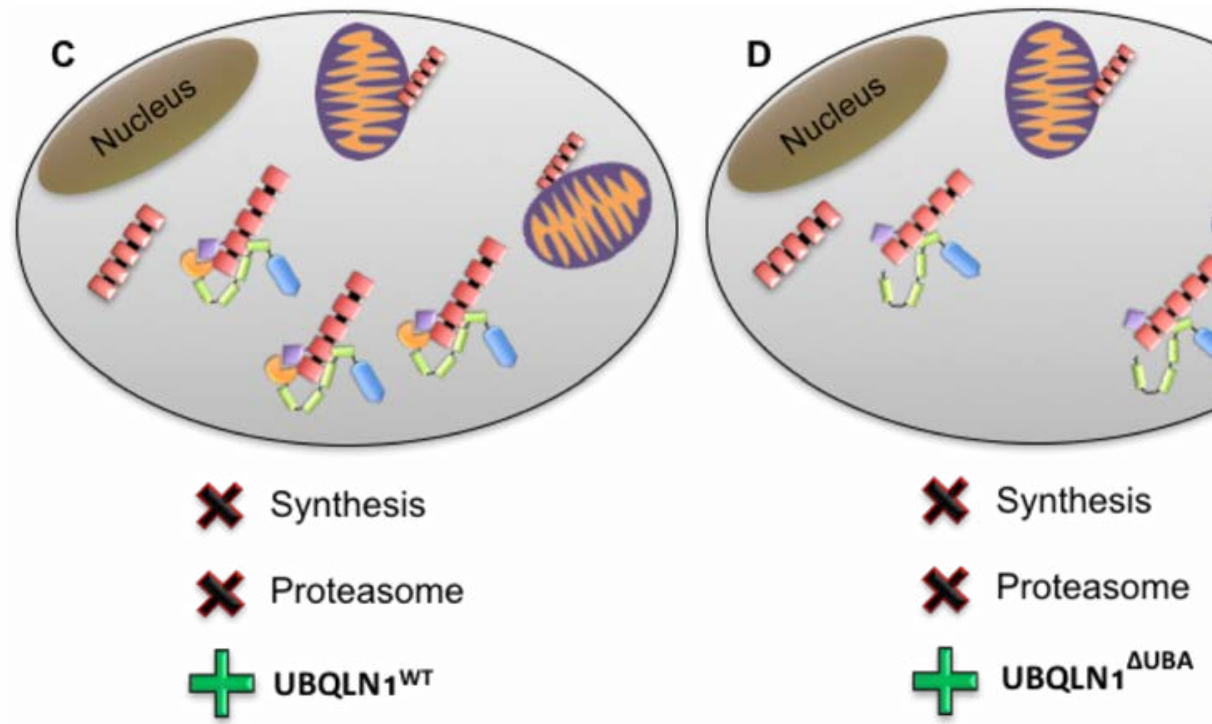

Figure 6: Schematic of mechanism of stabilization of BCLb by UBQLN1.

(A) Steady state expression of BCLb molecules in cells. BCLb is distributed in the cytoplasm and anchored in the mitochondrial outer membrane. (B) BCLb protein expression is lost within 16 hours of blocking new protein synthesis or blocking the proteasome. (C) When UBQLN1 is overexpressed, loss of BCLb due to mechanisms in $B$, is prevent and BCLb expression is stabilized overall. (D) UBQLN1 $1^{\triangle \mathrm{UBA}}$ cannot prevent loss of BCLb as UBQLN1 ${ }^{\mathrm{WT}}$. 
A

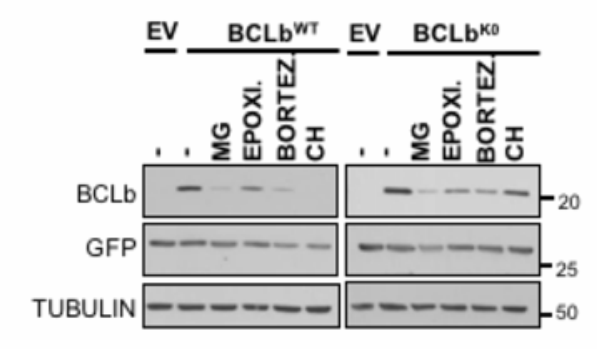

C

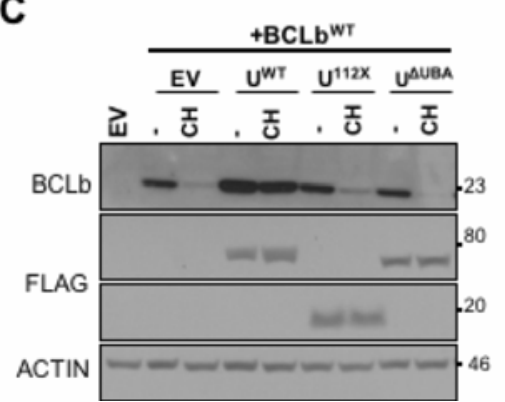

B

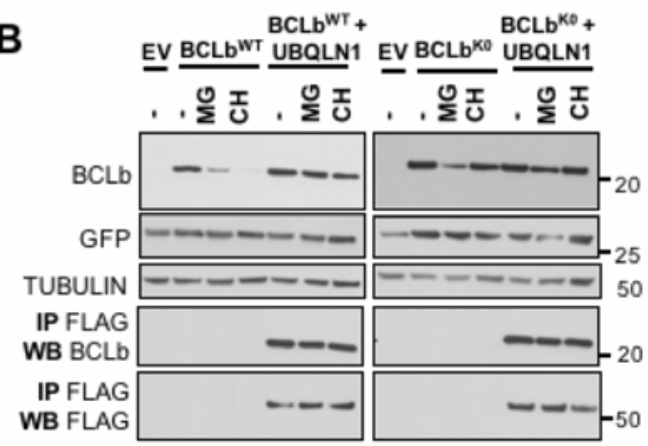

D

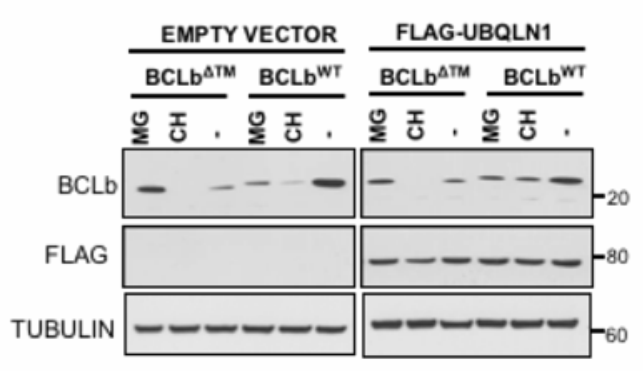

Figure 7: UBA domain of UBQLN1 is responsible for stabilization of $\mathrm{BCLb}^{\mathrm{WT}}$.

293T cells were transfected with MIG-BCLb ${ }^{W T}$, MIG-BCLb ${ }^{W T}, M^{W I G-B C L b}{ }^{\Delta T M}$, FLAG-UBQLN1 ${ }^{\mathrm{WT}}$, domain deletion constructs FLAG-UBQLN $1^{112 X}$ and FLAG-UBQLN1 ${ }^{\triangle U B A}$ as indicated. 36 hours post transfection, cells were treated with the indicated inhibitor for 16 hours; vehicle (-), translational elongation inhibitor Cycloheximide $(\mathrm{CH})$ and proteasomal inhibitors MG132 (MG), Epoximicin (Epoxi.) and Bortezomib (Bortez.). Cells were lysed and Western Blot analyses were performed with the indicated antibody. (A) Stability of $B C L b^{W T}$ and $B C L b^{K 0}$ was tested in the presence of UBQLN1, when exposed to the indicated inhibitors for 16 hours. (B) Experiment was performed as in A but in the presence of UBQLN1. As seen in A, expression of $B C L b^{W T}$ is lost upon exposure to $\mathrm{CH}$ for 16 hours, but in the presence of UBQLN1, it is stabilized. Loss of BCLb ${ }^{W T}$ seen with proteasomal inhibitors is also rescued by the presence of UBQLN1. UBQLN1 also prevents loss of $B C L b^{K 0}$ seen with MG132. (C) Experiment was performed as in $A$ in the presence of $\mathrm{CH}$ and domain deletion constructs of UBQLN1 and we mapped the UBA domain to be responsible for stabilizing BCLb ${ }^{W T}$. Both $\mathrm{UBQLN}^{112 \mathrm{X}}$ and UBQLN1 ${ }^{\triangle \mathrm{UBA}}$ failed to prevent loss of BCLb ${ }^{\mathrm{WT}}$. (D) Stability of $B C L b^{\triangle T M}$ was tested similar to $B C L b^{W T}$ and $B C L b^{K 0}$. BCLb ${ }^{\triangle T M}$ is generally less stable than BCLb ${ }^{W T}$ and presence of UBQLN1 does not alter its expression. (These experiments were performed twice). 
IGF1R, ESYT2 and BAG6 are newly identified substrates of UBQLN1. Interaction of UBQLN1 with IGF1R and ESYT2 is STI-mediated while with BAG6 and PSMD4 is UBL-mediated

We characterized the nature of interaction between UBQLN1 and BCLb using BCLb as a model substrate. We wanted to determine if these interaction characteristics could be generalized to other substrates of UBQLN1. Our IP/MS data (not shown) identified insulin-like growth factor 1 receptor (IGF1R), a cell surface receptor tyrosine kinase, Extended Synaptogammin-2 (ESYT2), a calcium regulated intrinsic membrane protein and BAG6, a protein involved in proteasomal degradation machinery of misfolded proteins, as potential interacting partners of UBQLN1. We mapped the interaction of UBQLN1 with IGF1R and ESYT2 to the STI domains and BAG6 and PSMD4 (or S5a, $26 S$ proteasomal subunit and known interacting partner of UBQLN proteins) to the UBL domain (Fig. 8A). Like with BCLb, STI domains of UBQLN1 are required for interaction with IGF1R and ESYT2 as UBQLN1 ${ }^{112 X}$ missing the STI domains is unable to interact with these 2 substrates. Interaction of UBQLN1 with BAG6 and PSMD4 is purely UBL mediated as detected with all 3 constructs, UBQLN1 $1^{\mathrm{WT}}$, UBQLN $1^{112 \mathrm{X}}$ and UBQLN1 $1^{\triangle \mathrm{UBA}}$ that have the UBL domain intact. Like BCLb, UBQLN1 interacts with IGF1R and ESYT2 via its STI domains but needs the UBA domain to stabilize it after proteasomal inhibition

Once we identified additional substrates that interact with different domains of UBQLN1, we wanted to determine substrate stability with specific interaction domains (Fig. 8B). We performed stability experiments with IGF1R, ESYT2, PSMD4 and BAG6 as we did with BCLb. Cells were transfected with empty vector (EV), UBQLN1 ${ }^{\mathrm{WT}}$, UBQLN $1^{112 X}$ or UBQLN $1^{\triangle \mathrm{UBA}}$ and exposed to $\mathrm{CH}$ or MG132 for 48 hours. Our results show that IGF1R expression decreased in the presence of $\mathrm{CH}$, which blocks new protein synthesis. IGF1R expression also decreased in response to proteasomal inhibition by MG132, indicating that it is being degraded through proteasomal- 
independent pathways (Fig. $8 \mathrm{C}$ ). In the presence of UBQLN1 ${ }^{\triangle \mathrm{UBA}}$, degradation of IGF1R through these non-proteasomal pathways seemed to be accelerated indicating that the presence of UBA domain prevents the loss of expression of IGF1R with MG132 exposure. We found strikingly similar data when we tested stability of another membrane protein ESYT2. UBQLN1 ${ }^{\triangle \mathrm{UBA}}$ failed to prevent loss of IGF1R and ESYT2 under proteasomal blockade suggesting that the UBA domain of UBQLN1 is essential in stabilizing IGF1R and preventing its degradation through non-proteasomal pathways. PSMD4 and BAG6 levels seemed steady and unchanging under both $\mathrm{CH}$ and MG132 exposure and the presence of UBQLN1 $1^{\mathrm{WT}}$, UBQLN $1^{112 \mathrm{X}}$ or UBQLN1 $1^{\triangle \mathrm{UBA}}$ did not alter their expression suggesting that substrate interactions of UBQLN1 that are UBL mediated, are not stabilized as a result of their interaction. 


\section{A}

\begin{tabular}{|c|c|c|}
\hline \multicolumn{3}{|c|}{$\begin{array}{c}\text { Immunoprecipitation/Mass } \\
\text { Spectrometry }\end{array}$} \\
\cline { 1 - 2 } $\begin{array}{c}\text { Unique Peptide } \\
\text { Count }\end{array}$ & \multirow{2}{*}{$\begin{array}{c}\text { Gene } \\
\text { Name }\end{array}$} \\
\cline { 1 - 2 } EV & $\begin{array}{c}\text { MIG- } \\
\text { UBQLN1 }\end{array}$ & \multirow{2}{*}{ UBQLN1 } \\
\cline { 1 - 2 }- & 26 & IGF1R \\
\hline- & 10 & ESYT2 \\
\hline- & 30 & PSMD4 \\
\hline- & 7 & BAT3/BAG6 \\
\hline- & 34 & \\
\hline
\end{tabular}

B

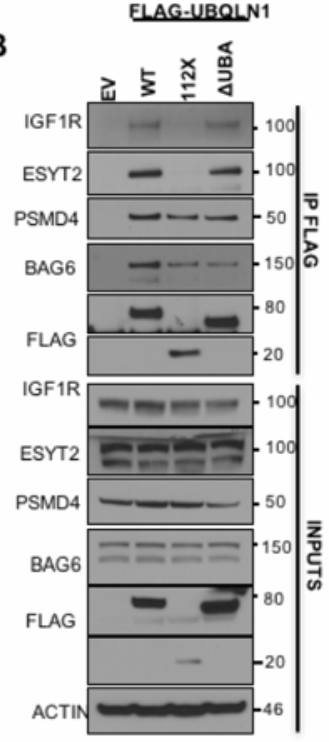

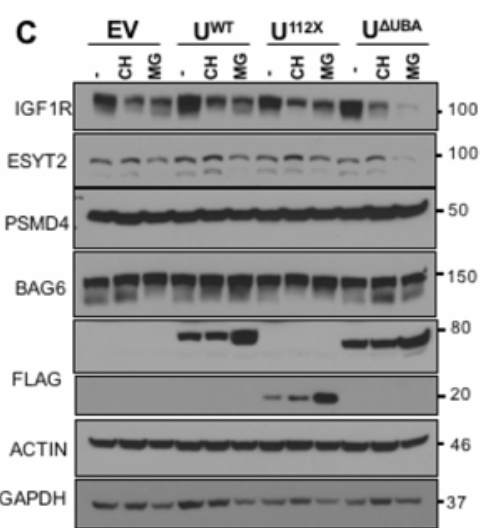

Figure 8: Fate of substrates targeted by UBQLN1 is dependent on interaction of the substrate with specific domains of UBQLN1.

(A) 293T cells were transfected with FLAG-tagged UBQLN1 (MIG-PLIC1) and an empty vector control (EV) followed by immunoprecipitation and analysis by mass spectrometry. The data from one representative experiment is shown. The number of unique peptides identified for each UBQLN1-interacting protein is shown. (B) 293T cells were transfected with FLAG-tagged deletion constructs of UBQLN1 followed by immunoprecipitation by FLAG antibody and Western Blot analysis. Like $\mathrm{BCLb}^{\mathrm{WT}}$, STI domains of UBQLN1 are required to interact with IGF1R and ESYT2 as UBQLN $1^{112 X}$ missing the STI and UBA domains do not interact with these two substrates. UBQLN1 ${ }^{\mathrm{WT}}$, UBQLN1 $1^{112 \mathrm{X}}$, and UBQLN1 ${ }^{\triangle U B A}$ interact with both PSMD4 and BAG6 indicating that this interaction is UBL mediated. (C) Stability of IGF1R, ESYT2, PSMD4, and BAG6 were tested upon $\mathrm{CH}$ and MG132 exposure for $48 \mathrm{~h}$ as with BCLb in Fig. 4. In the absence of the UBA domain (UBQLN1 ${ }^{\triangle U B A}$ ), IGF1R and ESYT2 expression are lost upon proteasomal inhibition with MG132. PSMD4 and BAG6 expression are unchanged after $48 \mathrm{~h}$ of $\mathrm{CH}$ and MG132 and the presence of UBQLN $1^{\mathrm{WT}}$, UBQLN $1^{112 \mathrm{X}}$, or UBQLN $1^{\triangle \mathrm{UBA}}$ does not affect their stability.(These experiments were performed twice). 


\section{UBQLN1 is capable of homodimerization through its STI-4 domains}

Several studies have reported that UBQLN1 is capable of homodimerizing (19) and UBQLN2 heterodimerizes with UBQLN4 (20). Feng (15) and Ford (19) showed that the middle region with STI domains is responsible for dimerization while other studies suggest a UBL-UBA dependent interaction. We investigated the presence of UBQLN1 dimers and determined if it is capable of interacting with substrates in its dimerized state. Cells were transfected with empty vector (EV), FLAG-UBQLN1 ${ }^{\mathrm{WT}}$ or deletion constructs of FLAG-UBQLN $1^{\mathrm{WT}}$ and MYC-UBQLN1 ${ }^{\mathrm{WT}}$. Results show MYCUBQLN1 was pulled down when we immunoprecipitated FLAG- UBQLN1 and the STI domains located between amino acid 112 and 542 in UBQLN1 are required for this interaction to take place (Fig. 9A). We mapped this interaction specifically to the STI4 domain (Fig. 9B). Our data do not indicate a UBL- UBA interaction, as recently suggested (18). These data indicate that FLAG-UBQLN1 and MYC-UBQLN1 associate with each other. However, whether this complex is between 2 or more molecules of UBQLN1 cannot be answered by these data. It is possible that UBQLN1 participates in a multimeric complex with other molecules of UBQLN1 or its isoforms. Next, we investigated if interaction with substrate disrupted UBQLN1 dimers (Fig. 9C). Results show that endogenous substrates of UBQLN1 (IGF1R; lanes 2,6,7) or introducing exogenous substrates (BCLb and IGF1R; lanes 7 and 8 respectively) do not alter the amount of dimerized UBQLN1 protein as witnessed by a consistent amount of MYC-UBQLN1 being pulled down with FLAG-UBQLN1. This suggests that the dimers do not need to be disrupted for interaction with substrates. 


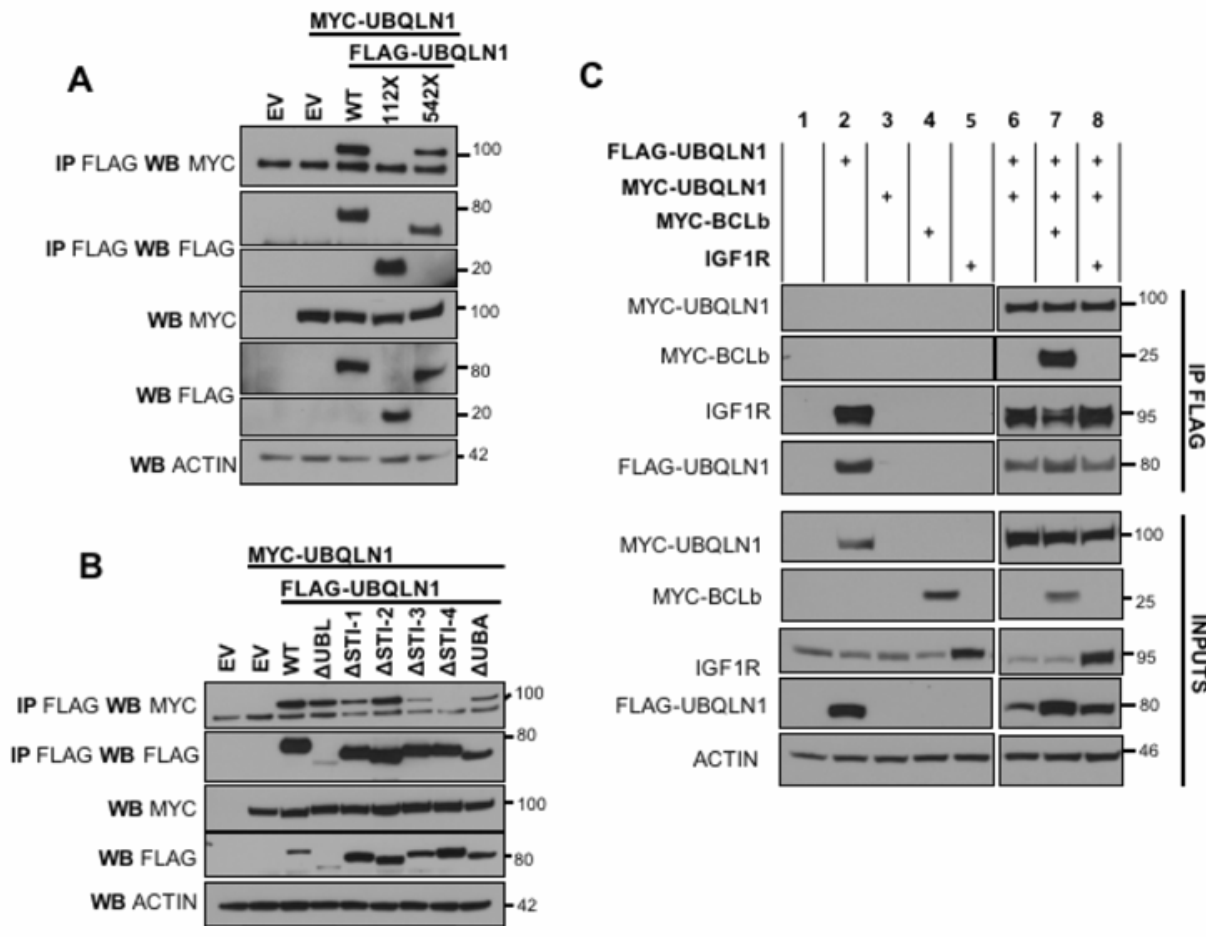

Figure 9: UBQLN1 is capable of homodimerization through its STI4 domains

UBQLN1 dimerizes via its STI-4 domains. A total of 293T cells were transfected with empty vector (EV), MYC-UBQLN1 ${ }^{\mathrm{WT}}$, FLAGUBQLN1 ${ }^{W T}$, domain deletion constructs of UBQLN1 MYC$U B Q L N 1^{W T}$ as indicated. Forty-eight hours post transfection, cells were lysed and Western Blot analyses were performed with the indicated antibody. Where indicated, immunoprecipitation with anti-FLAG conjugated agarose beads was performed to determine interaction. (A) FLAG-UBQLN1 ${ }^{\mathrm{WT}}$ interacts with MYC-UBQLN1 and the STI domains located between amino acid 112 and 542 are required for this interaction to take place. (B) Domain deletion constructs map the dimerization to the STI-4 domain. (C) The level of dimerization of UBQLN1 proteins remain unchanged in the presence of endogenous substrates (IGF1R; lanes 2,6,7) and introducing exogenous substrates (BCLb and IGF1R; lanes 7 and 8, respectively). (These experiments were performed twice). 


\section{DISCUSSION}

In this study we aimed to identify characteristics of UBQLN1-substrate interaction that lead to proteostasis of the substrate. Using BCLb as a model substrate, our data challenge the dogma that the primary function of UBQLN1 is to facilitate degradation of misfolded proteins. We undertook a systematic approach to investigate indicators of stabilization of BCLb as a substrate of UBQLN1.

'First, we investigated whether diverse ubiquitin linkages on proteins that interact with UBQLN1 play a role in its proteostasis. We used domain deletion constructs of UBQLN1 and confirmed that its UBA domain associates with ubiquitin (Fig. 3B,C). Further, we found no difference in the type of ubiquitin linkage recognized by the UBA domain when we overexpressed HA-Ubiquitin constructs capable of forming $\mathrm{K} 33, \mathrm{~K} 48$, $\mathrm{K} 63$ poly-ubiquitin chains as well as mono-ubiquitinating substrates (Fig. 3D). Our data complement findings by Zhang et al (17) that the UBA domain of UBQLN1 binds to both monomeric ubiquitin as well as poly-ubiquitin chains. Feng et al (15) also reported that the UBA domain is capable of interacting with poly-ubiquitin chains but did not test for interaction with monomeric ubiquitin. We have previously shown that BCLb lysine residues get mono- ubiquitinated and van de Kooji et al (21) showed that one out of its 4 lysines, K128 residue on BCLb is capable of forming K48 poly-ubiquitin chains which signals BCLb for proteasomal degradation. Our data indicate that the UBA domain non-selectively interacts with ubiquitin on BCLb, prevents binding of additional ubiquitin molecules that signal BCLb for proteasomal degradation (Fig. 3,4,7). Thus, unlike K48 ubiquitination, which specifically signals for degradation or K63 ubiquitination, which signals for trafficking, the ubiquitin code on UBQLN1's substrates does not decide its fate. To further understand the characteristics of UBQLN1-BCLb interaction, we mapped the location of their interaction on both proteins. We identified the STI-1/2 domains on UBQLN1 and transmembrane domain (TM) of BCLb as essential for this association to take place 
(Fig. $4 A, B$ ). UBQLN1 most likely recognizes the TM domain as BCLb is being translated and binds to it, stabilizing BCLb in the cytoplasm. We performed several stability experiments of BCLb in the presence of translational and proteasomal inhibitors (Fig. 7). BCLb ${ }^{\mathrm{WT}}$ is degraded completely following 16 hours of cycloheximide $(\mathrm{CH})$ treatment. UBQLN1 prevents loss of BCLb implying that their physical interaction protects $B C L b$ from degradation. In the presence of $C H, U B Q L N 1^{\triangle U B A}$ interacts with BCLb via its STI-1/2 domains (Fig. 4A) but fails to stabilize it (Fig. $7 C$ ) indicating that the UBA domain is required to stabilize BCLb. Lysine-less $B C L b\left(B C L b^{K 0}\right)$ is more stable than BCLb ${ }^{\mathrm{WT}}$ at 16 hours post cycloheximide treatment as our group (2009) and van de Kooji et al in 2013 have previously shown. Added stability of BCLb ${ }^{\mathrm{K} 0}$ over $B C L b^{W T}$ suggests that degradation is dependent on its lysines and therefore, likely its ubiquitination. van de Kooji et al showed that BCLb gets K48 poly-ubiquitinated on its $\mathrm{K} 128$ residue, which signals $\mathrm{BCLb}$ for proteasomal degradation. We hypothesize that when UBQLN1 is overexpressed, its UBA domain acts as a ubiquitin receptor and blocks chain elongation past mono-ubiquitination, thus preventing formation of K48 ubiquitin chains and degradation.

We tested whether UBQLN1-induced stability observed with BCLb could also be a phenomenon for other substrates of UBQLN1. Our IP/MS data (not shown) and IP/WB data (Fig. 8A) indicate that UBQLN1 interacts with insulin-like growth factor 1 receptor (IGF1R) and Extended Synaptogammin-2 (ESYT2) and like BCLb, it interacts with these 2 membrane proteins through its STI domains. We tested stability of IGF1R and ESYT2 (Fig. 5B) under similar conditions as with BCLb (Fig. 7). Upon blocking the proteasome with MG132, IGF1R and ESYT2 expression is lost and most likely due to degradation via proteasomal-independent pathways. However, in the presence of UBQLN1, its loss is prevented. UBQLN1 $1^{112 X}$ and UBQLN1 $1^{\triangle U B A}$, both missing the UBA domain, fail to protect IGF1R and ESYT2 from degradation. We found that as with BCLb, the UBA domain of UBQLN1 is responsible for stabilizing IGF1R and ESYT2. 
Recently, Suzuki et al (22), showed that UBQLN4 is a component of the multi-protein proteasomal machinery complex involving BAG6 and is responsible for recognition of the exposed transmembrane domains of newly synthesized defective polypeptides, eventually leading to their

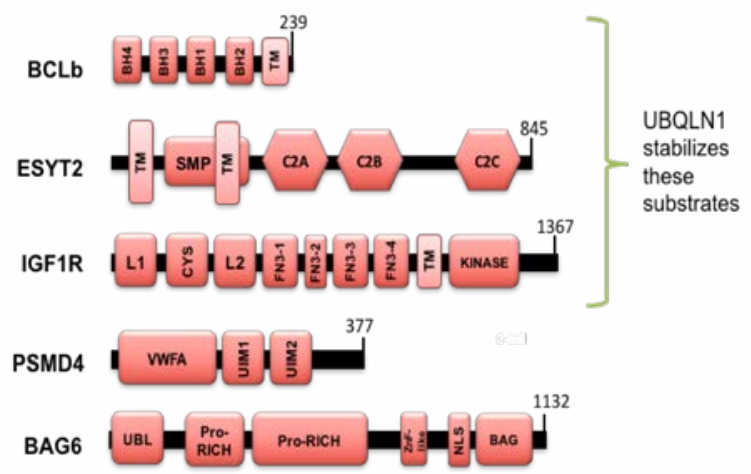

Figure 10: Schematic showing domains of substrates UBQLN1 interacts with. Substrates with transmembrane domains (TM) such as BCLb, IGF1R, ESYT2 are stabilized as a result of their interaction. proteasomal degradation. We UBL-mediated interactions with PSMD4 found that BAG6 interacts with the UBL domain of UBQLN1 and also confirmed PSMD4 to be an interacting partner of UBQLN1 (Fig. 8A). As UBL-interacting substrates of UBQLN1, we examined whether UBQLN1 binding affects stability of PSMD4 and BAG6 (Fig. 8B). We tested this under similar conditions as with BCLb, IGF1R and ESYT2 and found that UBQLN1 does not regulate proteostasis of PSMD4 and BAG6 (Fig. 8B). We detected no differences in the stability of PSMD4 and BAG6 in the presence UBQLN1 ${ }^{\mathrm{WT}}$, UBQLN $11^{112 X}$ and UBQLN1 $1^{\triangle \mathrm{UBA}}$ when treated with translational or proteasomal inhibitors.

Several studies have addressed the role of UBA domain of UBQLN1 in stabilizing proteins. Mah et al (14), showed that the UBA domain of UBQLN1 binds Presenilin1 (PSEN1) and Presenilin2 (PSEN2) proteins. Massey et al (13) confirmed that the UBA domain binds PSEN1/2 and stabilizes these proteins by preventing its poly-ubiquitination and proteasomal degradation. Haapaslo et al (23) found that overexpression of UBQLN1 stabilized higher molecular weight PSEN1, preventing its proteasomal enodproteolysis into smaller N-terminal fragment (NTF) and C-terminal 
fragment (CTF). Other substrates that have been reported to be stabilized by the UBA domain of UBQLN1 are p53 and IKBa. Overexpression of UBQLN1/2 prevented degradation of $p 53$ by E6AP ubiquitin ligases and IKBa by SCF ${ }^{\beta T R C P}$ ubiquitin ligase complex by blocking additional ubiquitination (24).

Currently, there is little consensus on the working model of UBQLN1. Our data

\section{Current model UBQLN1-Substrate-Proteasome interaction}
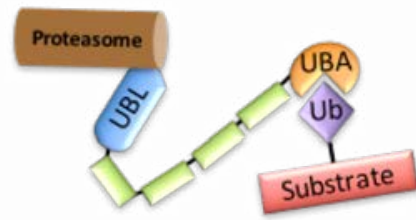

\section{Proposed Model}

Substrate Stabilization Model

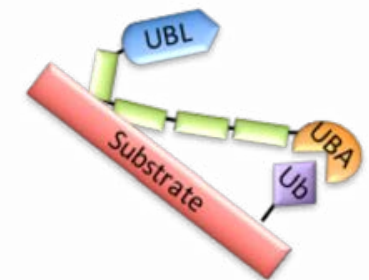

Figure 11: Current Model: UBQLN1 interacts with substrates through the UBA domain and with the proteasome through the UBL domain, directing it for degradation.

Proposed Model: UBQLN1 interacts with transmembrane proteins through its STI domains and secondary association through the UBA domain which stabilizes it and prevents its untimely loss.

suggest that UBQLN1 is capable of forming dimers via its STI- 4 domain (Fig. 9A,B) and is active in its dimerized state as it continues to interact with exogenous BCLb as well as endogenous and exogenous IGF1R (Fig. 9C). Our dimerization data confirm the mapped location of dimerization shown by Feng (15) (region 441-589) and Ford (19) referring to the STI-4 domain (Fig. 9B) as middle regions, i.e. not the $\mathrm{N}$-terminal UBL or C-terminal UBA. Hjerpe et al (20) suggest that when not associated with substrates, UBQLN2 exists in a UBL-UBA conformation or in an inactive resting state. They hypothesize that UBQLN1 opens up and adopts a monomer form to execute its function. The UBL-UBA dimer hypothesis subsists because the UBL domain is essentially like the ubiquitin molecule, but this domain shares only 4 lysines (K6, K11, $\mathrm{K} 27, \mathrm{~K} 48$ ) out of 7 (other three $-\mathrm{K} 29, \mathrm{~K} 33, \mathrm{~K} 63$ ) found in a ubiquitin molecule which may contribute to its decreased affinity for the UBA domain. The UBL domain of UBQLN1 has been shown to interact with proteins involved in the proteasomal 
machinery and not just UBA containing proteins. hHR23a is a UBL- UBA protein in this class that adopts a closed conformation due to a UBL-UBA interaction When UBL of hHR23a binds to the proteasomal subunit S5a, the closed conformation is disrupted (25). However, hHR23a has a UBL domain with 11 lysines and 2 UBA domains and an XPC region for DNA excision repair. Functionally and location wise, UBQLN1 is different from hHR23a. UBQLN1 is a largely cytosolic protein, its UBL domain has fewer lysines and one UBA domain and it is probably ill advised to compare functional dynamics of UBQLN1 to another UBL-UBA protein.

Current model of UBQLN1 suggests that UBA domain of UBQLN1 binds to poly-ubiquitin chains of substrate and shuttles it to the proteasome via its UBL domain for degradation (Fig. 11). Our data indicate that UBQLN1 is capable of interacting with substrates via STI domains (BCLb, IGF1R, ESYT2), UBL domain (PSMD4, BAG6), and UBA domain (ubiquitin). Kleijnen et al (26) showed that not only the UBL domain but also UBA domain on UBQLN2 is capable of directly binding to the S5a cap of the proteasome. The ability of the UBA domain to bind the proteasome is unique to UBQLN proteins as UBA domain of $\mathrm{c}-\mathrm{CBL}$ is unable to bind to the proteasome (26). Therefore, it is possible that the role of UBQLN1's UBA domain is to bind to ubiquitin on substrates and shield them from being recognized for degradation. The UBA domain of UBQLN1 is a highly-conserved region across different species and across different isoforms of UBQLN1 protein (UBQLN1-4, UBQLNL). For over a decade, UBQLN1 has been shown to assist in degradation of proteins but our data suggest that UBQLN1 also stabilizes some proteins that it binds to, like BCLb, IGF1R and ESYT2. We propose a substrate-stabilization model of UBQLN1 (Fig. 11) such that primary association of UBQLN1 with substrate occurs through the STI domains and a secondary association through its UBA domain, which leads to stabilization of the substrate. We do not know whether primary associations through the UBL domain result in degradation of substrate but it does not appear to stabilize the substrates it binds to. We conclude 
that fate of substrates that UBQLN1 associates with, is interaction-domain specific. All 3 interacting partners of UBQLN1 stabilized by the UBA domain are membrane proteins; BCLb - mitochondrial membrane protein, IGF1R - plasma membrane protein, ESYT2 - endoplasmic reticulum/plasma membrane protein (27). We identified the transmembrane domain of BCLb to be essential to be recognized by UBQLN1. Thus, it is a plausible hypothesis that UBQLN1 recognizes proteins with

\begin{tabular}{|c|c|c|c|}
\hline $\begin{array}{c}\text { UBL mediated } \\
\text { interactions }\end{array}$ & $\begin{array}{c}\text { STI mediated } \\
\text { interactions }\end{array}$ & $\begin{array}{c}\text { UBA mediated } \\
\text { interactions }\end{array}$ & $\begin{array}{c}\text { Indirect } \\
\text { interactions }\end{array}$ \\
\hline
\end{tabular}

Figure 12: Models of Interaction of UBQLN1

This schematic shows hypothetical mechanisms of interaction of UBQLN1 with its substrates through it's individual domains.

transmembrane domains via its STI domains and stabilizes them via its UBA domain.

UBQLN1 is a known stress response protein and protects cells from heat shock and hypoxic stress (20) and it seems reasonable that UBQLN1 stabilizes crucial survival proteins like BCLb, IGF1R and p53 over others. Future work will involve characterizing the mechanisms involved in regulation of IGF1R expression and activity by UBQLN1 and investigating whether UBQLN1 can be targeted in disorders resulting from IGF1R dysregulation. 


\section{CHAPTER 4}

\section{INTRODUCTION}

\section{Insulin-like Growth Factor-1 Receptor}

IGF1R (Insulin-like Growth Factor-1 Receptor) is a receptor tyrosine kinase ubiquitously expressed on cell surfaces of all tissues. Ligand stimulation causes the receptor to transmit signals to stimulate cell proliferation, differentiation, survival and cellular metabolism. The transmembrane IGF receptors exist in the plasma membrane as preformed dimers. Known ligands of IGF1R are IGF-1 (highest affinity), IGF-2 and insulin and stimulate IGF1R to activate PI3-AKT and RAS-MAPK downstream pathways among others. IGF binding proteins 1-6 (IGFBPs) bind ligands and limit their bioavailability and binding to IGF1R (Kurlawala et al., 2017; Yarden, 2001).

The IGF pathway has been under investigation as an anti-cancer drug target for several decades since IGF1R expression was determined to be crucial for SV40 large tumor antigen to transform mouse embryonic fibroblasts and its activity was discovered to stimulate proliferation in vitro (Sell et al., 1993). IGF1R over expression is associated with an increased risk of recurrence of non-small cell lung cancer (NSCLC)(Nakagawa et al., 2012). A meta-analysis of 17 studies comprising 3,294 NSCLC patients concluded that expression of IGF1R positively correlated with patients' smoking status, tumor size and negatively correlated with disease-free survival (S. Zhao, Qiu, He, Li, \& Li, 2014). Additionally, autocrine and paracrine production of IGF1 has been linked to an increased risk of development of breast, prostate, and colorectal cancers (Brahmkhatri, Prasanna, \& Atreya, 2015; Vigneri et al., 2015).

Acromegaly or gigantism, an endocrinopathy associated with high circulating levels of IGF1 is associated with three times increased risk of developing colorectal 
cancer (Renehan et al., 2003). In a contrasting condition called Laron dwarfism, patients have low circulating IGF1 levels and are resistant to development of cancer and diabetes (Laron, 2008).

The IGF1R gene does not harbor activating mutations like other receptor tyrosine kinases (EGFR and FGFR) and ligand independent activation of IGF1R is not known. Still, overexpression of IGF1R is a hallmark finding in lung cancer, malignant melanoma, primary breast cancer and pancreatic adenocarcinoma (Brahmkhatri et al., 2015). This implies that alterations in expression and activity of IGF1R can result from anomalous mechanisms of autocrine and paracrine stimulation of receptor, hybrid assemblies of IGF receptors, abnormalities in other proteins that regulate its trafficking and turnover, and even epigenetic and transcriptional control (Grimberg, 2003). For example, mutations in c-Cbl, an E3 ligase, can dysregulate turnover of c-MET, another receptor tyrosine kinase, leading to persistent signaling even in the absence of receptor overexpression or activating oncogenic mutations (Peschard et al., 2001). Currently, the IGF1/IGF1R axis is a major target of research for cancer therapy. However, several pharmacological agents -monoclonal antibodies and small molecule inhibitors, targeting this axis have failed to show significant benefit on overall survival (Janssen \& Varewijck, 2014). Unfortunately, expression of IGF1R does not always correlate with its cell surface expression and therefore it can be misleading to correlate its expression with expected response to therapy. This highlights the role of cellular and extracellular factors that modulate the activity of IGF1R directly or indirectly.

We have identified UBQLN1 as a regulator of IGF1R expression and activity. The Ubiquilin family of proteins (UBQLN1-4, UBQLNL) are evolutionarily conserved structurally similar to each other. UBQLN1 is approximately a $63 \mathrm{kDa}$ protein and has 3 main domains: ubiquitin-like domain (UBL) at the $\mathrm{N}$-terminus, ubiquitin-associated domain (UBA) at the C-terminus and STI-1 chaperone-like regions in the middle (Fig. 1A). UBQLN1 interacts with IGF1R and its UBA domain is required to stabilize IGF1R expression (Kurlawala et al., 2017). UBQLN1 is lost and under-expressed in $50 \%$ of 
lung adenocarcinomas and loss of either UBQLN1 or UBQLN2 promotes epithelial to mesenchymal transition (EMT) in lung adenocarcinoma cell lines (Shah et al., 2015). UBQLN1 is known to regulate other cell surface receptors like Presenilins (Mah et al., 2000), GABA $_{A}$ receptors (Saliba et al., 2008; Y. Zhang et al., 2015) and nicotinic acetylcholine receptors (Ficklin et al., 2005). Here, we demonstrate that UBQLN1 interacts with IGF1R, IGF2R and INSR and is essential for the normal expression and activity of IGF1R in lung adenocarcinoma cells.

IGF1R Structure and Trafficking: The IGF1R is synthesized as an immature pro-receptor monomer that undergoes maturation though proteolysis and glycosylation to form dimers. IGF1 receptors are present on the cell surface as preformed dimers unlike EGFR that form dimers upon ligand binding (Figure 3). The $\beta$ subunit consists of 627 amino acids, has a small extracellular portion, transmembrane portion and the intracellular C-terminal. The juxtamembranous region in the $\beta$ subunit hosts the NPXY motif and the catalytic region. The NPXY motif participates in the process of receptor internalization while the catalytic region is crucial for ATP binding. Tyrosine 1131, 1135 and 1136 in the intracellular tyrosine kinase domains are critical for receptor autophosphorylation. The intracellular domains hold crucial signals that decide the fate of receptor trafficking once it is activated. For example, even though overall there is $70 \%$ amino acid homology between IGF1R and Insulin receptor, there is only $44 \%$ homology in their $\mathrm{C}$-terminus sequence which dictates the differences in downstream signaling pathways and functions between the 2 receptors. The NPXY motif recognizes the phosphotyrosine binding domains (PTB) of IRS proteins and SH2 proteins (Figure 4). The IRS proteins (IRS 1-4) undergo full activation within 1-2 mins and Shc protein within 5-10 mins of ligand binding to IGF1R (Girnita et al., 2014). IRS proteins interact with IGF1R on the cell surface through their pleckstrin homology domains $(\mathrm{PH})$ and PTB domains are present in their $\mathrm{N}$-terminus. The $\mathrm{C}$-terminus region of IRS proteins are variable and control the variety of interactions with other signaling molecules. The C-terminal of IRS proteins have a high affinity for proteins 
that have an SH 2 domain like PI3K, Grb2, SH-PTP2 (phosphatase), adaptor proteins like CRK, NCK1 which act as docking proteins for interaction of IGF1R with other proteins like $\beta 1$ integrins on the cell surface. Like IRS proteins, SHC proteins (SHC AD) also consist of a PTB domain at their N-terminal and a SH2 domain at their Cterminal and interact with proteins in a similar fashion. These domains, their characteristics and the interactions they are capable of control further trafficking events of the activated receptor. Internalization of IGF1R is known to occur through clathrin mediated endocytosis (CME) and also through caveolin-mediated endocytosis at high levels of IGF1 (Goh \& Sorkin, 2013). Internalized RTK's in early endosomes proceed to late endosomes and continue to send signals through their C-terminus in the cytoplasm. In the late endosome, the RTK either gets recycled back to the plasma membrane through a recycling endosome or gets degraded via lysosomal or proteasomal pathways (Figure 5). Multi-protein complexes called Endosomal Sorting Complex Required for Transport (ESCRT) are critical for cellular transport of activated receptors (Goh \& Sorkin, 2013). These processes occur by systematic co-operation of a variety of adaptor proteins and in absence of an interacting partner can potentially disturb trafficking and alter biological consequences. Ubiquitin interacting motif (UIM) containing adaptor proteins like Epsin, Eps15, Eps15R recognize ubiquitin on RTKs, bind to clathrin and AP2 simultaneously and are critical for clathrin mediated endocytosis (CME) of some RTKs like EGFR (Figure 6). The NPXY motif not only regulates internalization of IGF1R but also its downregulation. All RTKs possess a ubiquitin binding motif and ubiquitination has been well established as a crucial regulatory process of RTK trafficking. Ubiquitin is a small protein $(7 \mathrm{kD})$ that is added to IGF1R in 3 consecutive steps via the E1, E2 and E3 ligases. The first two enzymes, E1 and E2 work to load the last enzyme E3 on to IGF1R to add ubiquitin molecules on the receptor. IGF1R is a known substrate of three E3 ligases: Mdm2 (Girnita, Girnita, \& Larsson, 2003) Nedd4 (Vecchione, Marchese, Henry, Rotin, \& Morrione, 2003) and c-Cbl (Sehat, Andersson, Girnita, \& Larsson, 2008). Ubiquitination of IGF1R occurs 
before entering the endocytic vessels during internalization (Vecchione et al., 2003). Mdm2 poly-ubiquitinates IGF1R with K63-type chains and $\beta$-arrestins have been identified as crucial adaptor proteins to recruit Mdm2 to IGF1R (Girnita et al., 2005). Similarly, Grb10 is a key adaptor protein that recruits Nedd4 to IGF1R which multi mono-ubiquitnates the receptor (Vecchione et al., 2003). At higher doses of IGF1, cCbl poly-ubiquitinates IGF1R with K48-type chains. Mdm2 recruitment favors stimulation of MAPK over PI3K pathways. Like IGF1R, EGFR is also poly-ubiquitinated with K63-type chains (Huang, Kirkpatrick, Jiang, Gygi, \& Sorkin, 2006). K48 polyubiquitination signals target substrates for proteasomal degradation while K63 monoand poly-ubiquitination are recognized by other proteins through their ubiquitin binding domains (UBD) and activate enzyme cascades like kinases, phosphatases, phospholipases and so on that feed into and activate multiple downstream pathways like PI3K/AKT, RAS/MAPK, metabolic pathways and others (Adhikari \& Chen, 2009; Varadan et al., 2004). Ubiquitination is a major molecular signal responsible for endocytic sorting such that ubiquitination of IGF1R is essential for its internalization while is not for other RTKs like EGFR and FGFR2. However, degradation of these 3 RTK's is highly dependent on their ubiquitin-conjugation (Haugsten, Malecki, Bjorklund, Olsnes, \& Wesche, 2008; Huang et al., 2006; Mao et al., 2011). Previously, we have reported that UBQLN1 interacts with IGF1R and UBQLN1's UBA domain is required to stabilize it. We have also published that UBQLN1 is lost and underexpressed in $50 \%$ of lung adenocarcinomas and loss of either UBQLN1 or UBQLN2 promotes epithelial to mesenchymal transition (EMT) in lung adenocarcinoma cell lines (Shah et al., 2015). Here, we demonstrate that UBQLN1 interacts with IGF1R, IGF2R and INSR and is essential for their normal expression and activity. 

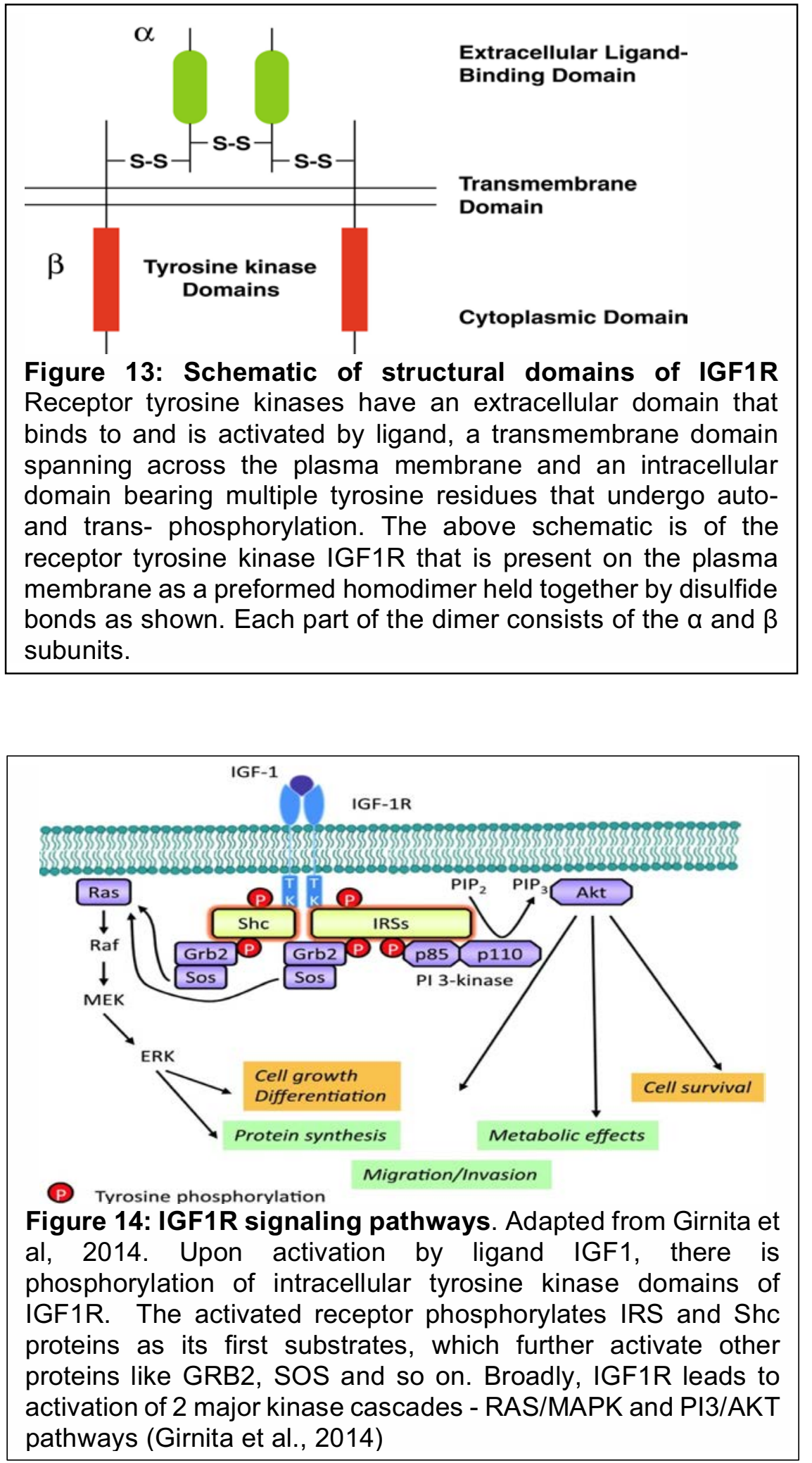


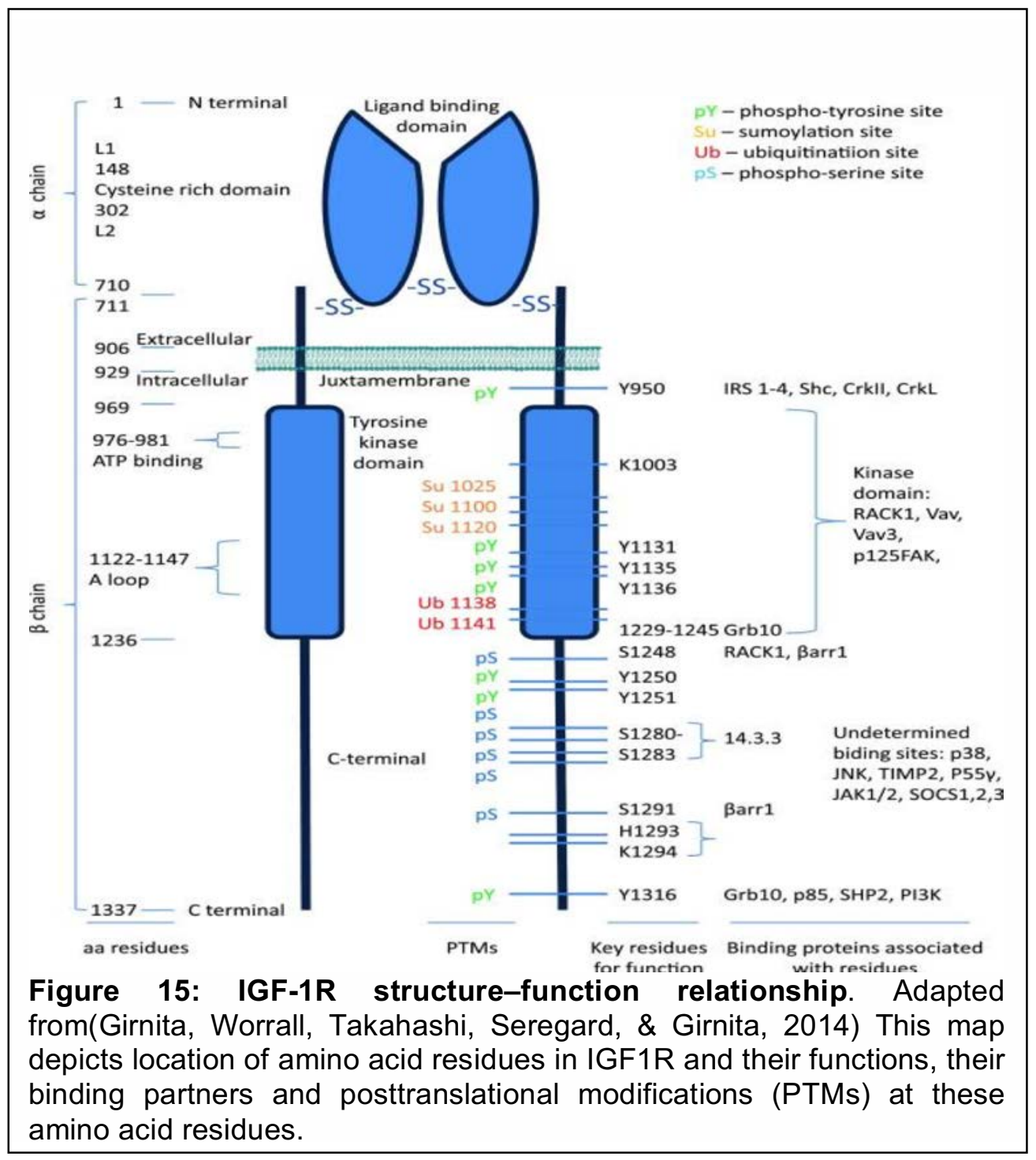




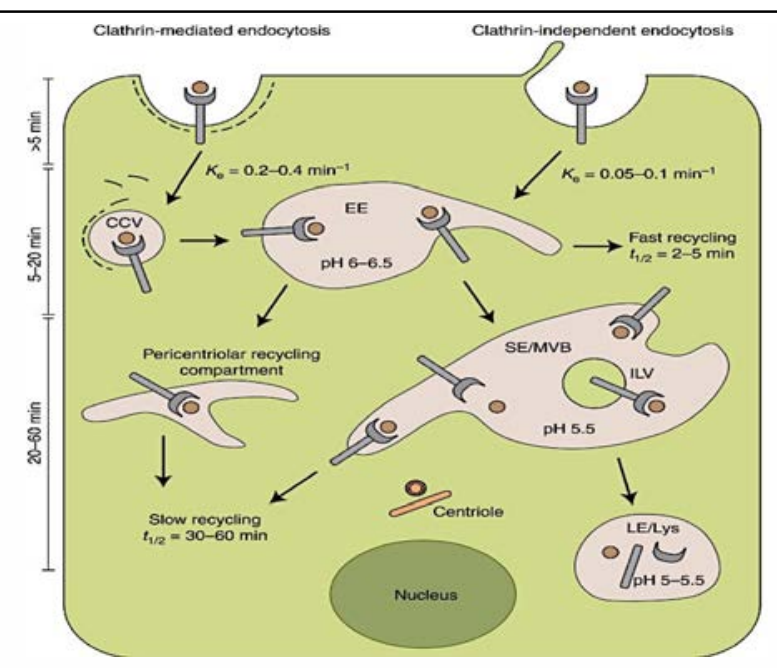

Figure 16: Endocytic Trafficking of RTK

Adapted from Goh \& Sorkin (Goh \& Sorkin, 2013). This figure shows 2 pathways of endocytosis of ligand bound RTK: clathrin mediated and calveolin mediated. The internalized receptor in clathrin-coated vesicles (CCV) is transported to the early endosome (EE) to the sorting endosome (SE) or the mutivescicular body (MVB) and from here either to the recycle endosome to be recycled back to the plasma membrane or degraded in the lysosomal endosome (LE).

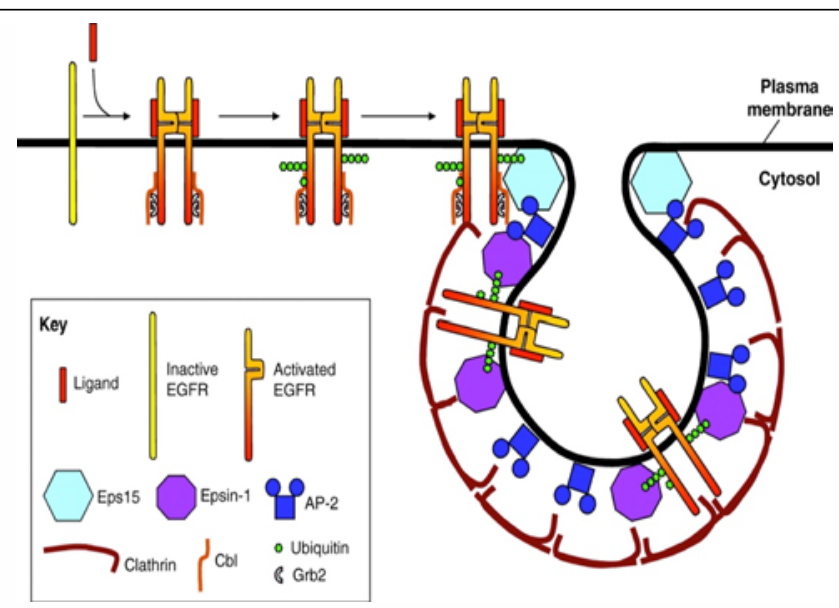

Figure 17: Schematic of clathrin-mediated endocytosis of EGFR. Adapted from Madshus et al (Madshus \& Stang, 2009) Phosphorylated RTK and activates tyrosine kinases facilitating binding of an E3 ligase $\mathrm{Cbl}$ to the C-terminal of the RTK, or indirectly via an adaptor protein Grb2. Poly-Ub chains added by Cbl interact with Ub-interaction motifs (UIMs) of Epsin-1 and Eps15 which act in co-ordination with other proteins (not shown) and subsequently cause invagination of the plasma membrane and form a clathrin-coated vesicle 


\section{RESULTS}

\section{UBQLN1 interacts with IGF1R, IGF2R, and INSR}

We recently demonstrated that UBQLN1 interacts with IGF1R and UBQLN1's UBA domain is required to protect IGF1R from MG132 (proteasomal inhibitor) mediated degradation (Kurlawala et al., 2017). Fig.18A shows the schematic of UBQLN1. We confirmed endogenous interaction between UBQLN1 and IGF1R by Immunoprecipitation/Western Blot (IP/WB) analysis (Fig. 18B,C). In addition to IGF1R, UBQLN1 also interacts with IGF2R and INSR. We used IGF1R as a model receptor for future experiments. To determine IGF1R and UBQLN1 co-localize in cells, FLAGUBQLN1 was overexpressed in HEK 293T cells followed by immunofluorescence (Fig. 18D). After adjusting for image saturation by JACop plugin of ImageJ software analysis, $33.5 \%$ of FLAG-UBQLN1 was determined to overlap with endogenous IGF1R, calculated by the Costes method and Mander's correlation coefficients. These immunofluorescence data imply that a fraction of total UBQLN1 in cells associates with IGF1R and support our recently published findings that UBQLN1 is available for interaction with other proteins and thus, is capable of participating in a variety of cellular processes (Kurlawala et al., 2017). 

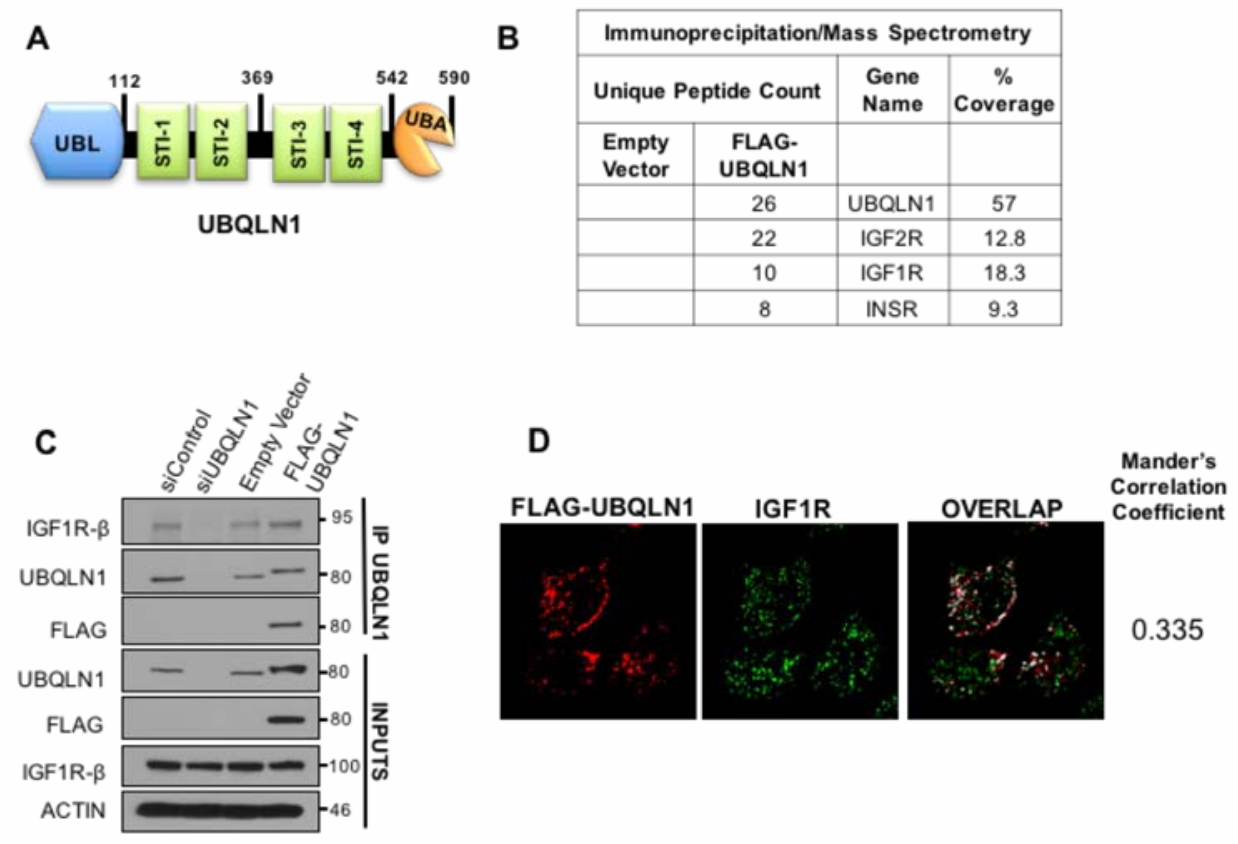

Figure 18: UBQLN1 interacts with IGF1R: A) Schematic of structure of UBQLN1 ${ }^{\text {wt }}$ protein. N-terminal UBL (Ubiquitin-Like) domain, C-terminal UBA (Ubiquitin-Associated) domain and $4 \mathrm{STI}-1$ domains in between. B) Immunoprecipitation (IP) of overexpressed FLAG-tagged UBQLN1 followed by mass spectrometry (MS) analysis to identify FLAG conjugated proteins. MIG is the empty vector used as a control for overexpression. IGF1R, IGF2R and INSR were some of the top interacting partners of UBQLN1. C) HEK293T cells were transfected with either control siRNA (siNT) or siRNA against UBQLN1 (siUBQLN1) to knock down UBQLN1 and empty vector (control) or FLAG-tagged UBQLN1 (FLAG-UBQLN1) to overexpress UBQLN1, followed by immunoprecipitation by UBQLN1 antibody and Western Blot analysis. Interaction was detected between endogenous UBQLN1 and IGF1R which was absent when UBQLN1 was absent (siUBQLN1) and slightly increased with UBQLN1 overexpression. (D) Confocal microscopy images of indirect immunofluorescence staining for FLAG-UBQLN1 (red) and IGF1R (green) in HeLa cells. Co-localization was determined for using JACop plugin of ImageJ software. Automatic threshold for images were determined by the Costes method and overlap coefficients (Mander's Correlation Coefficients) were calculated. For the chosen field, 33.5\% of FLAG-UBQLN1 overlaps with IGF1R. (Experiments were performed twice) 
UBQLN1 interacts with pro-form, phosphorylated and non-phosphorylated forms of IGF1R

Next, we mapped the domains of UBQLN1 required for interaction with IGF1R using a series of domain deletion constructs previously described (Kurlawala et al., 2017). As we showed with another transmembrane protein BCLb (Kurlawala et al., 2017), we found that the STI1 and STI2 domains of UBQLN1 are crucial for interaction with IGF1R. We examined cellular distribution of these constructs (FLAG tagged UBQLN1 ${ }^{\mathrm{WT}}$, UBQLN1 ${ }^{\triangle U B L}$, UBQLN1 $1^{\triangle S T I-1}$ and UBQLN1 ${ }^{\triangle \mathrm{UBA}}$ ) (Fig. 19A) in HEK 293T cells by immunofluorescence to confirm that overall distribution of UBQLN1 protein missing individual domains did not change (Fig. 19A). All constructs of UBQLN1 showed uniform distribution in the cytoplasm and all were distinctly absent from nuclei and vacuoles. STI-1 and STI-2 domains are responsible for interaction with IGF1R (Fig. 19B). We conducted additional interaction studies with overexpressed UBQLN1 in serum-free conditions devoid of receptor ligands and stimulation with exogenous IGF1 (Fig. 19C). Interestingly, UBQLN1 interacts with phosphorylated IGF1R, detected when the receptor is stimulated with exogenous IGF1, and this interaction disappears when phosphorylation is inhibited by Linsitinib, a specific small molecule inhibitor of IGF1R activity. UBQLN1 also interacts with non-phosphorylated IGF1R as detected in cells devoid of receptor stimulation (serum-free media) as well as complete media, serum-free media supplemented with IGF1 and in the presence of Linsitinib. Additionally, UBQLN1 interacts with the larger, pro-form of IGF1R detected at 135 kD (Fig. 19D). Based on our interaction data, we conclude that UBQLN1's association with IGF1R is independent of its phosphorylation status and it appears that UBQLN1 is recruited to the receptor from the time of its synthesis. 


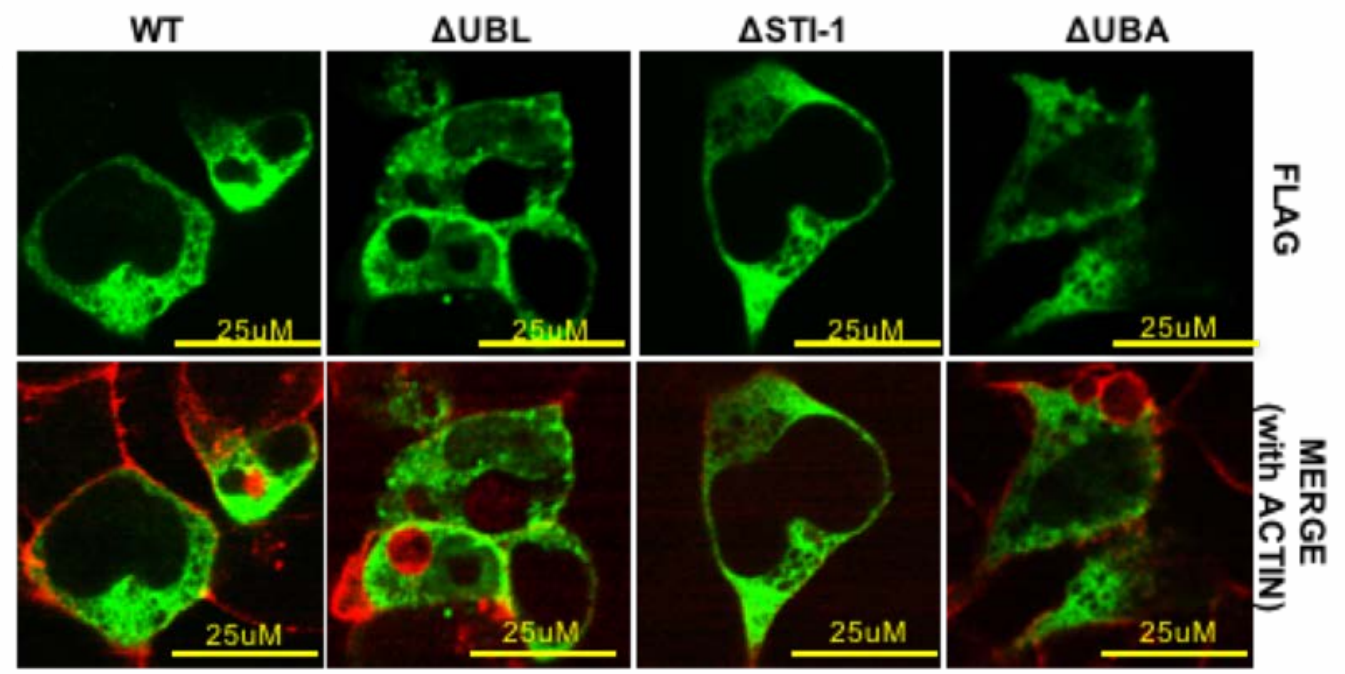

Figure 19: UBQLN1 interacts with pro-form, phosphorylated and nonphosphorylated forms of IGF1R

(A) HEK 293T cells were transfected with FLAG-UBQLN1 ${ }^{\triangle U B L}$, FLAG-UBQLN1 ${ }^{\triangle S T I-}$ 1 and FLAG-UBQLN1 $1^{\triangle \mathrm{UBA}}$ constructs followed by indirect immunofluorescence of FLAG-UBQLN1. UBQLN $1^{\mathrm{WT}}$ is distributed in the cytoplasm of transfected cells and is absent from nuclei and vacuoles. UBQLN1 $1^{\triangle \mathrm{UBL}}$, UBQLN1 $1^{\triangle \mathrm{STI}-1}$ and UBQLN1 ${ }^{\triangle \mathrm{UBA}}$ show similar cellular expression. (Experiment was performed once) 
B

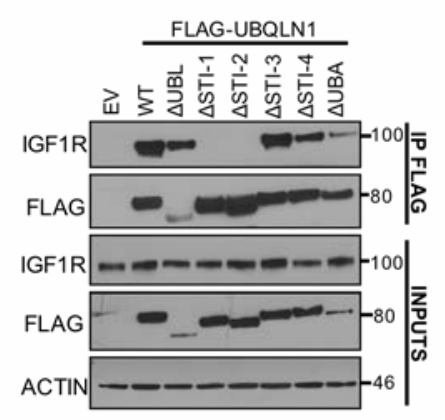

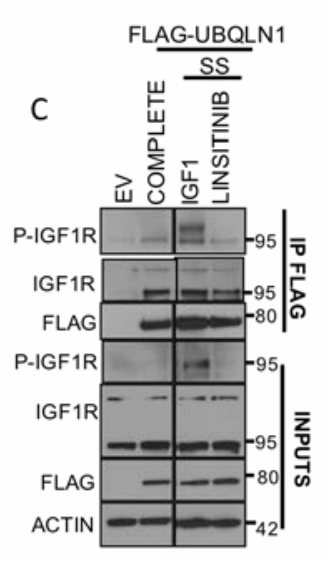

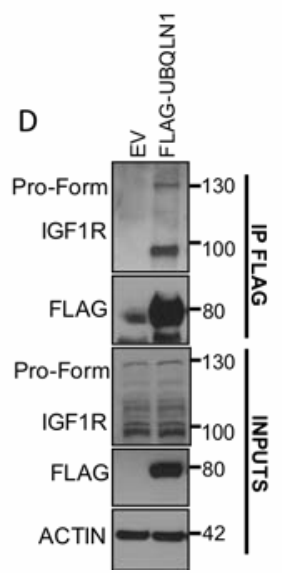

Figure 19: UBQLN1 interacts with pro-form, phosphorylated and nonphosphorylated forms of IGF1R

(B) HEK 293T cells were transfected with FLAG-tagged constructs of UBQLN1 (in A) followed by co-immunoprecipitation and Western Blot analysis. STI-1 and STI-2 domains of UBQLN1 are required for interaction with IGF1R. (C) HEK 293T cells were transfected with empty vector or FLAG-UBQLN1 and cells were cultured in complete media (COMPLETE) or serum-free media supplemented with IGF1 (SS, IGF1) or Linsitinib (SS, LINSITINIB), a small molecule inhibitor of IGF1R activity, followed by co-immunoprecipitation and Western Blot analysis. UBQLN1 interacts with phosphorylated and nonphosphorylated IGF1R. (IGF1:50ng/ml, Linsitinib:1uM). (D) HEK 293T cells were transfected with empty vector or FLAG-UBQLN1 showed that FLAGUBQLN1 interacts with both immature pro-form of IGF1R at 130kD and mature, processed IGF1R at 100kD. (Experiments were performed twice). 
UBQLN1 regulates expression and activity of IGF1R in lung adenocarcinoma cells. Following confirmation of interaction between UBQLN1 and IGF1R, we tested effects of loss of UBQLN1 on steady state expression of IGF1R in A549 cells, a non-small cell lung adenocarcinoma cell line. UBQLN1 protein expression was downregulated using two different siRNAs for UBQLN1 (U1 KD\#1 and U1 KD\#2). A549 cells were cultured in 2 different conditions: serum-free media for 12 hours and serum-free media (12 hours) supplemented with IGF1 (6 hours). Cells were incubated with Cycloheximide for an hour before adding IGF1 to block de novo protein synthesis, to allow assessment of steady state expression of IGF1R. Post-stimulation, cells were harvested, lysed and analyzed by Western Blot for total and phosphorylated IGF1R expression levels. While total IGF1R expression was decreased in UBQLN1 deficient cells in both conditions (Fig. 20A), differences were more pronounced post-stimulation with IGF1. Phosphorylated IGF1R levels were undetectable in serum free media, however, post stimulation with IGF1, the ratio of phosphorylated to total IGF1R levels was greatly increased in siUBQLN1 cells $(2.4$ and 2.2 times) compared to control (Fig. 20B). 

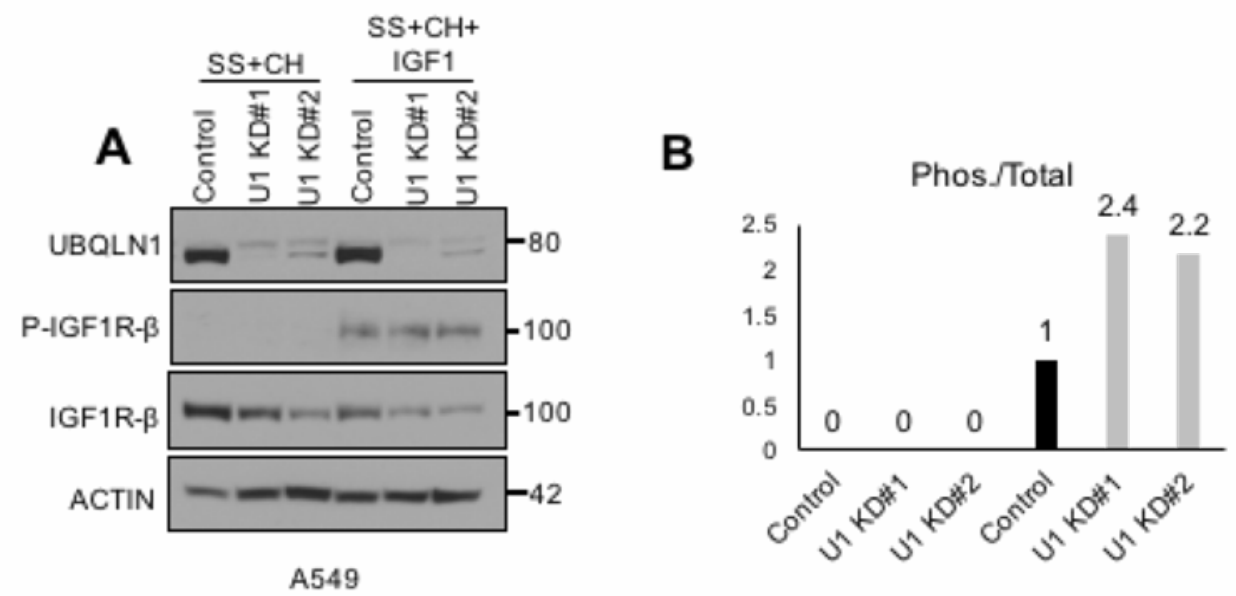

Figure 20: UBQLN1 regulates expression and activity of IGF1R

(A) Expression and activity of IGF1R were tested in A549 lung cancer cells following downregulation of UBQLN1 with two different siRNA (U1 KD\#1 and U1 KD\#2). Cells were serum starved (SS) overnight (12 hours), incubated with protein synthesis inhibitor Cycloheximide one hour prior to supplementing serum-free media with IGF1. 6 hours later, cells were harvested and lysed analyzed by Western Blot. UBQLN1 knock down cells showed decrease in total IGF1R expression compared to control in both conditions. Post-stimulation with IGF1, phosphorylated IGF1R levels were detected in both control and UBQLN1 deficient cells, however, the ratio of phosphorylated to total IGF1R levels was greatly increased in UBQLN1 deficient cells (2.4 and 2.2 times compared to control) as seen in the densitometry graphs in (B). Data are normalized to non-targeting siRNA control in unstimulated cells. (Experiments were performed thrice). 


\section{Loss of UBQLN1 results in decreased cell surface expression of IGF1R}

Following Western Blot data that showed decreased total IGF1R expression in cells, saturation binding assays were performed to test if the overall decrease in total IGF1R expression also reflected as a decrease in receptor number on the cell surface. Radioligand binding assays were performed utilizing radioactive ligand $\left(I^{125}\right.$-IGF1) to test for differences in the number of binding sites (number of cell surface receptors) in HPL1D cells between UBQLN1 deficient cells and control (Fig. 21). In these experiments, HPL1D cells were transfected with UBQLN1 siRNA (siU1) or control (siNT) and 48 hours post-transfection, cells were incubated with increasing concentrations of the radiolabeled ligand (I25-IGF1) with the intention to saturate the receptors. The purpose of performing saturation binding assays was to determine the differences in maximum binding capacity $B_{\max }$ (number of cell surface receptors) between UBQLN1 deficient cells and control. Plotting saturation curves showed that although the amount of radioactive ligand used did not saturate the receptors. Overall, number of cell surface IGF1 receptors were lower in UBQLN1 deficient cells while the Kd i.e. affinity is almost the same. Data are representative of one experiment. 


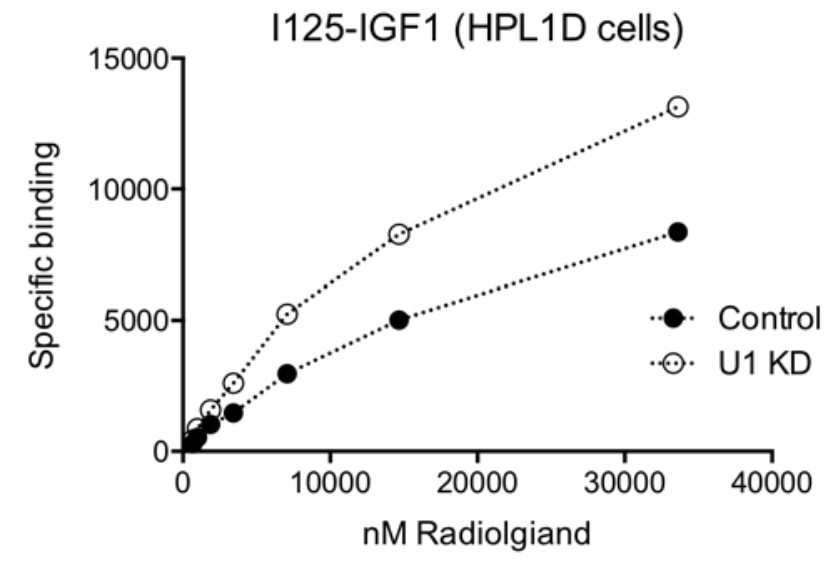

\begin{tabular}{|c|c|c|}
\hline & siNT & siUBQLN1 \\
\hline \multirow{2}{*}{ Bmax } & $16542 \mathrm{cpm}$ & $23149 \mathrm{cpm}$ \\
\cline { 2 - 3 } & $9600.47 \times 10^{-15} \mathrm{mmoles}$ & $\begin{array}{c}13,434.89 \times 10-^{15} \\
\mathrm{mmoles}\end{array}$ \\
\hline Receptors/cell & $46.955+/-16.779$ & $15.65+/-7.991$ \\
\hline Kd & $0.061+/-0.0077 \mathrm{nmol} / \mathrm{L}$ & $0.058+/-0.0035 \mathrm{nmol} / \mathrm{L}$ \\
\hline
\end{tabular}

Figure 21: Loss of UBQLN1 results in decreased cell surface expression of IGF1R

Specific Binding for $\mathrm{I}^{125}$-IGF1 for both control and UBQLN1 deficient cells are graphed. The table represents the differences in Bmax, Kd and number of receptors in HPL1D cells that have loss of UBQLN1. Bmax values are representative of one experiment for graphing purposes but receptor number and $\mathrm{Kd}$ values are calculated as average +/- standard error of mean from 3 individual experiments. Radioactivity was measured as counts per minute (CPM) in a Beckman gamma counter (efficiency=0.45). Specific activity of $\mathrm{I}^{125}$-IGF1 = $1870 \mathrm{Ci} / \mathrm{mmol}$. (Experiment was performed thrice. Data are representative of one individual experiment). 


\section{Loss of UBQLN1 accelerates loss of IGF1R}

After confirming that UBQLN1 regulates protein expression and phosphorylation activity of IGF1R at 6 hours post-stimulation with IGF1 (Fig. 21A), we asked whether loss of UBQLN1 altered recycling of IGF1R. Therefore, we examined cells at shorter and longer time points after IGF1 stimulation. A549 cells with stable expression of shRNA against UBQLN1 and control were treated with Cycloheximide, an inhibitor of de novo protein synthesis and tracked the expression and activity of IGF1R after IGF1 stimulation. We examined shorter time points, at intervals of 30 minutes from 0 to 180 minutes (Fig. 22A) and longer time points up to 24 hours (Fig. 22B) after adding exogenous IGF1. Overall, the pattern of phosphorylation of IGF1R between UBQLN1 deficient cells and control cells were almost identical. Based on our time points, IGF1R achieved peak phosphorylation by 30 minutes. While control cells maintained steady levels of total IGF1R throughout 180 mins of stimulation, its expression started to decline in UBQLN1 deficient cells by 90 minutes. This effect on IGF1R loss in UBQLN1 deficient cells was seen more clearly when we tracked receptor expression for 24 hours following IGF1 stimulation. While expression of IGF1R declined and begun to rise gradually in control cells, UBQLN1 deficient cells lost almost all IGF1R by 24 hours. Cycloheximide causes arrest of translational machinery thus preventing all new protein synthesis resulting from transcription. Therefore, it is likely that the increase in IGF1R expression observed in control cells is not due to increase in transcription but due to stimulation of Endoplasmic Reticulum (ER) and Golgi processing of pre-formed, already translated monomers to its mature dimerized form. UBQLN1 plays a role in stabilizing and assembling newly translated $\mathrm{GABA}_{\mathrm{A}}$ receptors in the ER (Saliba et al., 2008) and we hypothesize that it may do the same with IGF1R. Thus, it is possible that UBQLN1 deficient cells cannot replenish lost IGF1R like control cells do. 
A
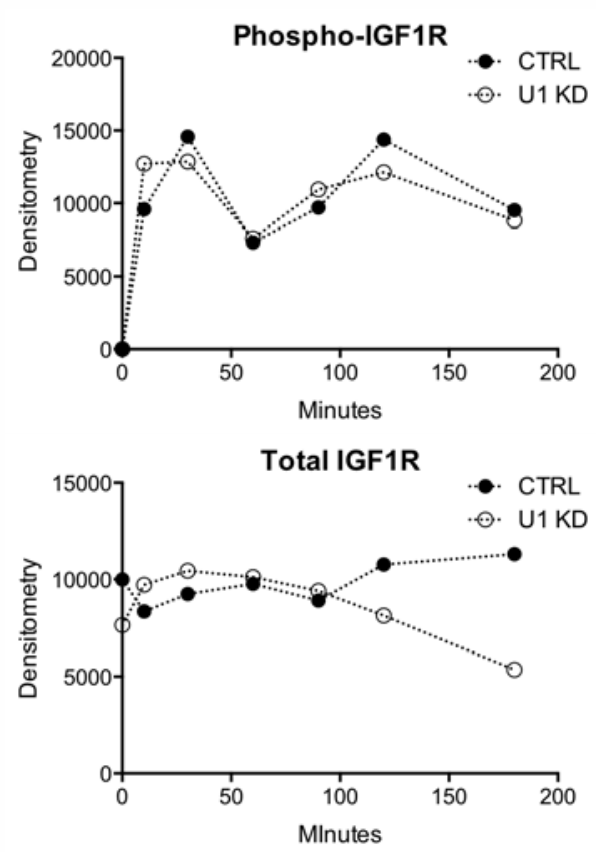

CTRL.

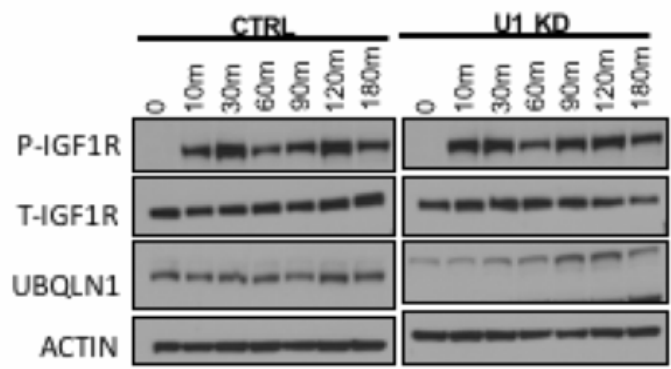

B
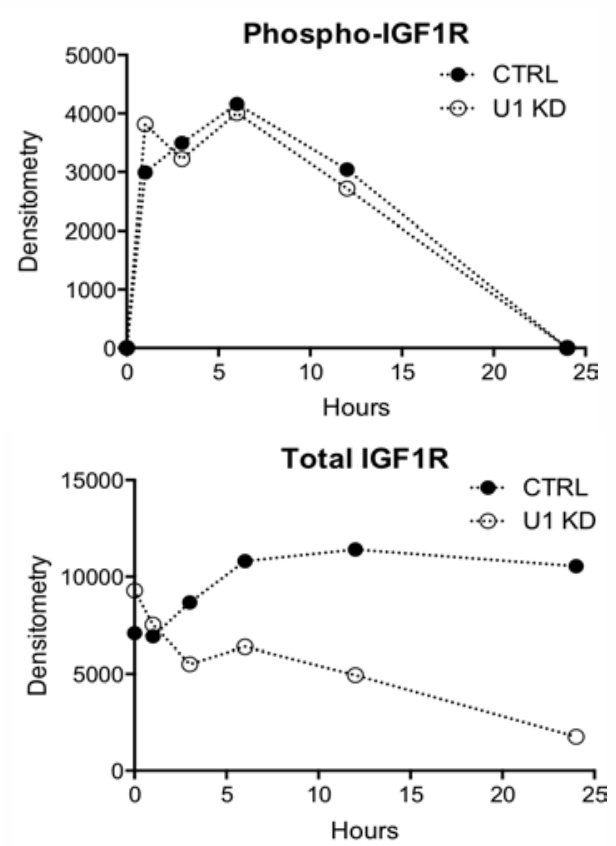

Figure 22: Loss of UBQLN1 accelerates loss of IGF1R

A549 cells expressing shRNA against UBQLN1 were incubated with Cycloheximide (20uM), an inhibitor of de novo protein synthesis to study loss of IGF1R expression in UBQLN1 deficient cells, post-stimulation with IGF1. Cells were harvested at the indicated time points and Western Blot analysis for phosphorylated and total receptor levels were performed and graphed. (A) Post-stimulation, cells were harvested at intervals of 30 mins from 0 to 180 minutes Western Blot analysis were performed with the indicated antibodies. (B) Longer time points until 24 hours were examined post-stimulation with IGF1. 


\section{Loss of UBQLN1 inhibits recycling of IGF1R}

Since UBQLN1 deficient cells have accelerated loss of IGF1R, we studied whether UBQLN1 altered recycling and degradation of the receptor. We used Monensin, an ionophore that traps the internalized receptor within early endosomes and prevents their recycling back to the cell surface. With vehicle treatment (Fig. 23A), UBQLN1 deficient cells have faster loss of IGF1R when compared to control. With Monensin treatment (Fig. 23B), expression of total IGF1R was parallel between control and UBQLN1 deficient cells. Interestingly, this pattern of IGF1R expression in Monensin treated cells imitated the pattern of IGF1R expression in vehicle treated UBQLN1 deficient cells. Based on these data, we conclude that loss of UBQLN1 has the same effect on IGF1R expression as Monensin does. This implies that loss of UBQLN1 may block recycling of IGF1R and cause accelerated degradation of internalized receptor trapped within endosomes. 

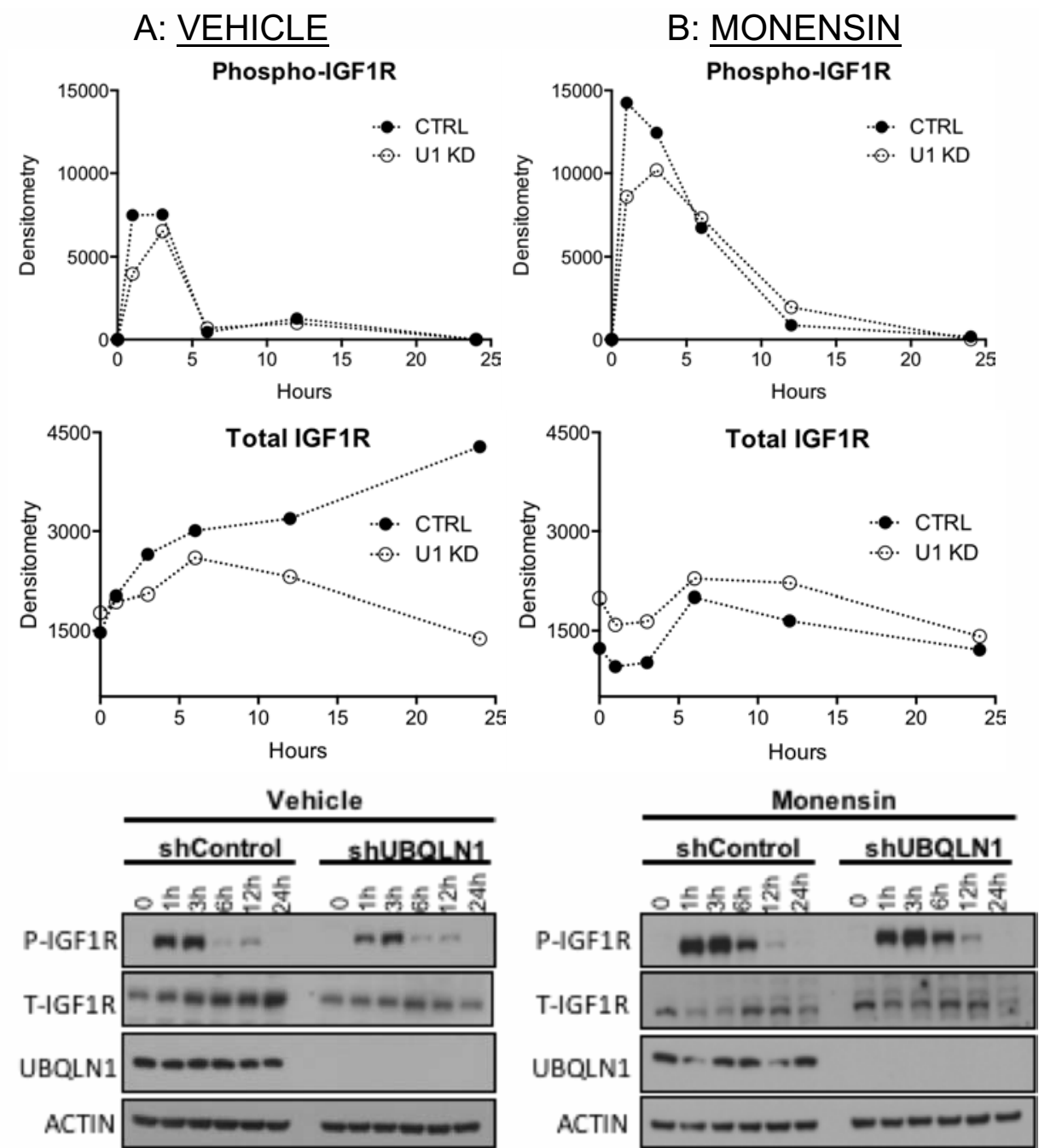

Figure 23: Loss of UBQLN1 inhibits recycling of IGF1R

A549 cells expressing shRNA against UBQLN1 were serum starved for 12 hours, incubated with Vehicle (A) or Monensin (B), inhibitor of early endosomes for an hour prior to stimulation with IGF1 and harvested post-stimulation at the indicated time points. Western Blot analysis for phosphorylated and total receptor levels were performed and graphed in both control and UBQLN1 deficient cells. Densitometry of Western Blot images were performed using Image J and PIGF1R and T-IGF1R expression were normalized to Actin expression in control cells for each treatment. (Experiment was performed twice for Monensin but once for Vehicle. Data representative of one complete vehicle and Monensin experiment). 


\section{UBQLN1 does not play a role in IGF1R turnover through the proteasome}

We conducted a similar experiment by inhibiting the proteasome with Bortezomib. As seen in vehicle (Fig. 24A), UBQLN1 deficient cells have lower expression of IGF1R, while expression in control cells steadily increases. Inhibiting proteasomal degradation with Boretzomib (Fig. 24B) prolonged the phosphorylation of IGF1R compared to vehicles and caused an initial accumulation of total IGF1R in UBQLN1 deficient cells which is eventually lost probably due to recruitment of other degradation pathways like the lysosome. There are no gross differences between vehicle and Bortezomib treatment in both control and UBQLN1 deficient cells. Based on these data, we conclude that UBQLN1 may not play a role in IGF1R turnover through the proteasome. 

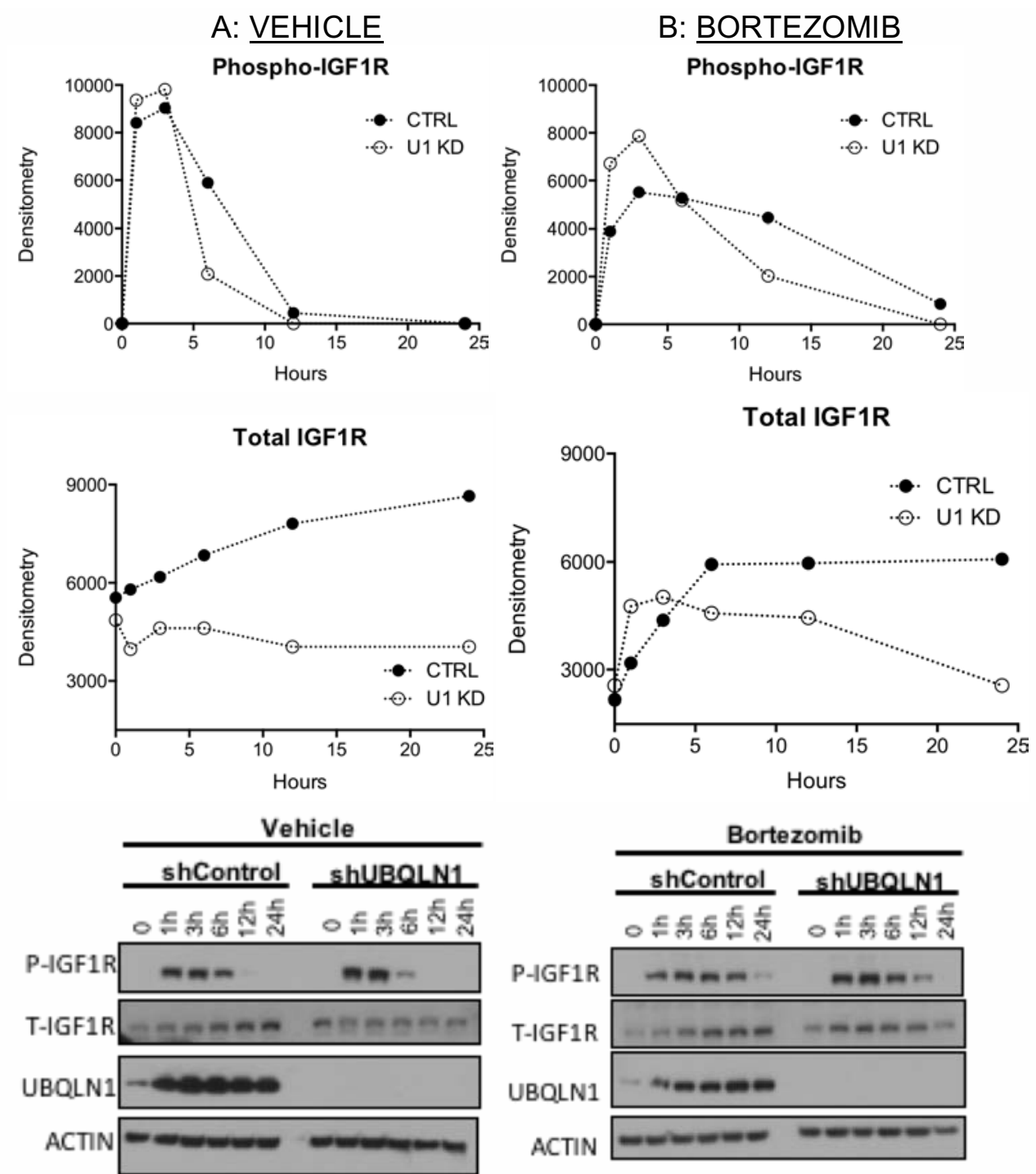

Figure 24: UBQLN1 does not play a role in IGF1R turnover through the proteasome

A549 cells expressing shRNA against UBQLN1 were serum starved for 12 hours, incubated with Vehicle (A) or Bortezomib (B), a proteasomal inhibitor for an hour prior to stimulation with IGF1 and harvested post-stimulation at the indicated time points. Western Blot analysis for phosphorylated and total receptor levels were performed and graphed. Densitometry of Western Blot images were performed using ImageJ and P-IGF1R and T-IGF1R expression was normalized to Actin expression in control. Experiment was performed twice for Bortezomib but once for Vehicle. Data representative of one complete vehicle and Bortezomib experiment. 


\section{Loss of UBQLN1 may direct IGF1R towards higher density compartments}

Next, we examined whether loss of UBQLN1 altered intracellular transport of IGF1R. To study gross differences in trafficking and organelle distribution of IGF1R in UBQLN1 deficient cells, A549 cells (control and shRNA against UBQLN1) were serum starved for 12 hours (Fig. 25A,C) and stimulated with IGF1 for 1 hour (Fig. 25B,D), harvested and cell lysates were loaded onto a Percoll density gradient. Every other fraction was resolved via SDS-PAGE and immunoblotted for markers of various organelles (EEA1=early endosomes, TfnR=plasma membrane/recycling/early endosomes). Note that an $R_{f}$ value of 1.0 represents the top of the gradient tube, while an $R_{f}$ value of 0 represents the bottom of the gradient. Therefore, higher density organelle associated markers like LAMP1 for late endosomes are detected in the higher gradients towards $R_{f=1.0}$ and lower density gradient associated markers like EEA1 for early endosomes are detected towards $R_{f=} 0$. Here, we probed for IGF1R, TfnR and EEA1. In UBQLN1 deficient cells, distribution of IGF1R and TfnR was slightly shifted towards higher density gradients like the lysosome compared to control. This indicates that loss of UBQLN1 may be altering trafficking of plasma membrane receptors as they get internalized from the plasma membrane 

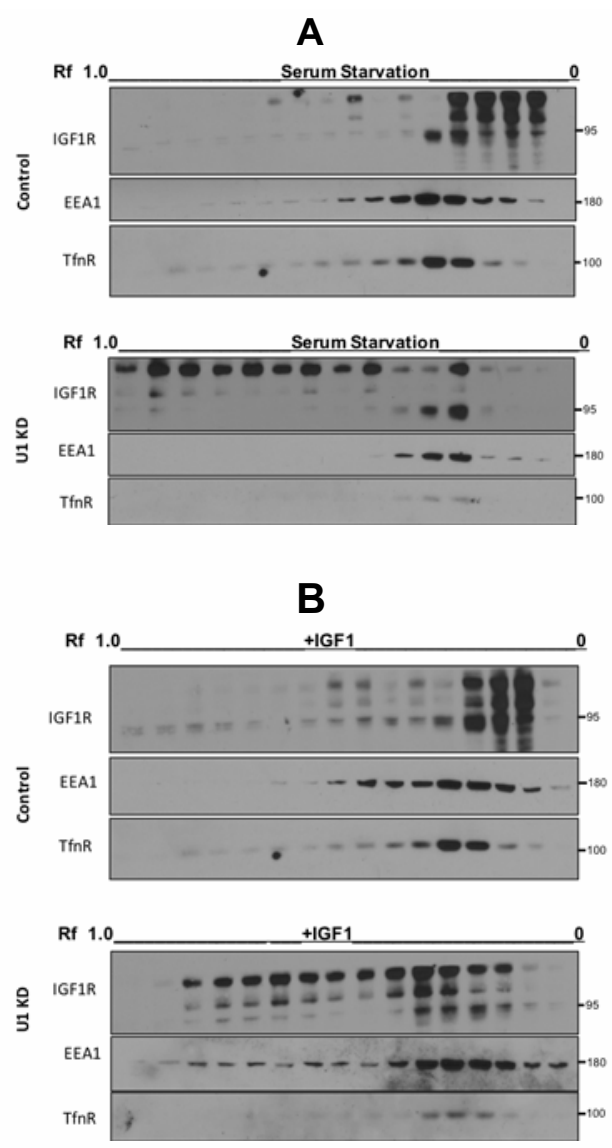

Figure 25: Loss of UBQLN1 may direct IGF1R towards higher density compartments

A549 cells (control and shRNA against UBQLN1) were serum starved (12 hours) (A) and stimulated with IGF1 for 1 hour (B), harvested and cell lysates were loaded onto a Percoll density gradient. Every other fraction was resolved via SDS-PAGE and immunoblotted IGF1R and for markers of various organelles (TfnR=transferrin receptor, plasma membrane/recycling/early endosomes, EEA1=early endosome antigen 1 , early endosomes). Note that an $R_{f}$ value of 1.0 represents markers associated with higher density gradients, while an $R_{f}$ value of 0 represents lower density gradients). (Experiment was performed thrice. Data are representative of one individual experiment). 


\section{C: SERUM STARVATION}

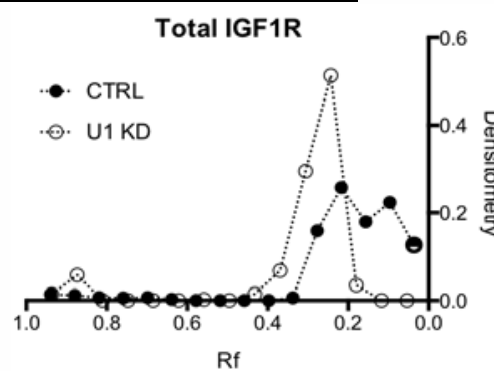

TnfR

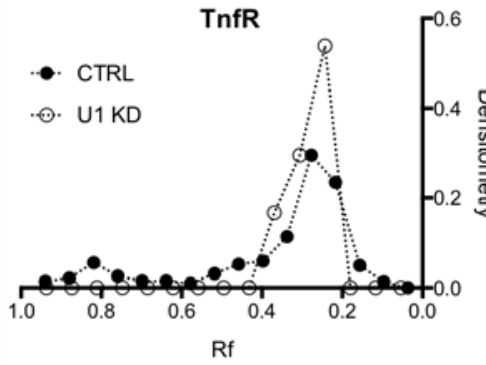

EEA1

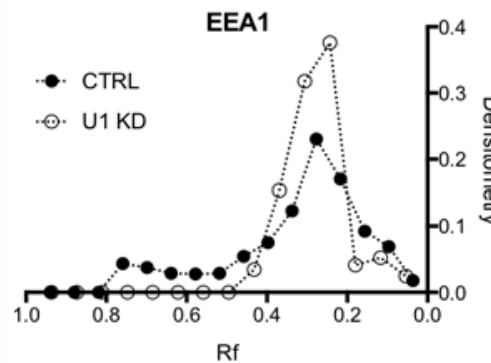

D: SERUM STARVATION+IGF1
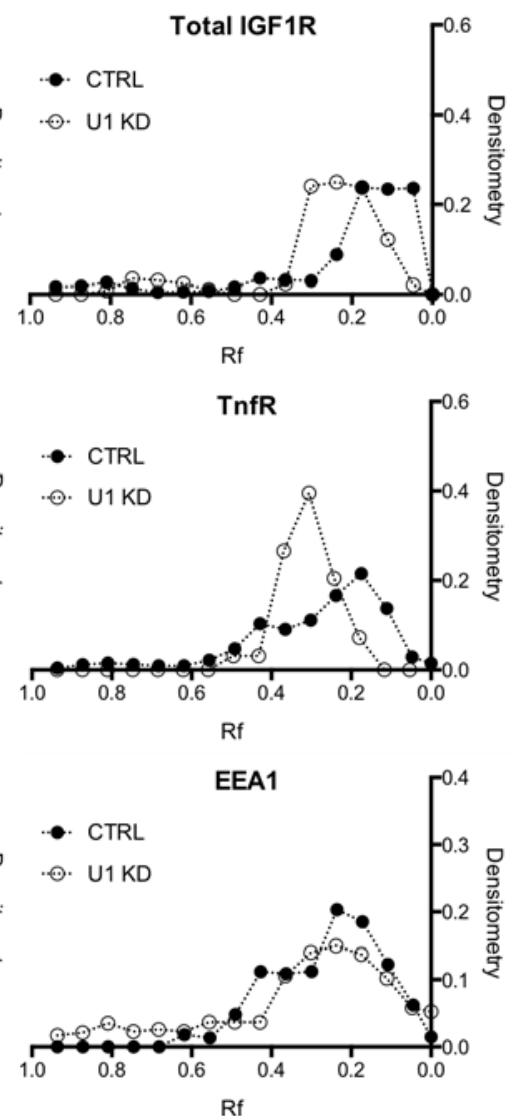

Figure 25: Loss of UBQLN1 may direct IGF1R towards higher density compartments

These graphs represent densitometry quantifications of Western Blot analysis $n$ both serum starved (C) and IGF1 stimulated (D) conditions, there is a slight leftward shift in distribution of IGF1R and TfnR in UBQLN1 deficient cells compared to control indicating that loss of UBQLN1 may be directing internalized cell surface receptors (IGF1R and TfnR) towards higher density compartments like lysosomes. 
UBQLN1 deficient A549 cells have increased survival in serum-free conditions A549 cells expressing stable shRNA against UBQLN1 (U1 KD\#1, U1 KD\#2, and control) were cultured in conditions of serum-free media and serum-free media supplemented with IGF1 for 4 days (Fig. 26). Alamar Blue readings were recorded every 24 hours. UBQLN1 deficient cells gradually begun to outsurvive control cells when cultured in serum-free media by Day 2 (Fig. 26A). Supplementing serum-free media with IGF1 (Fig. 26B) corresponded with a slight survival advantage in UBQLN1 deficient cells compared to control. Based on these data, we conclude that loss of UBQLN1 inherently confered a growth and survival advantage to A549 cells when cultured in serum-free media, and this effect is enhanced by stimulation of the IGF pathway by exogenous ligand. 
A

\section{Serum Starvation}

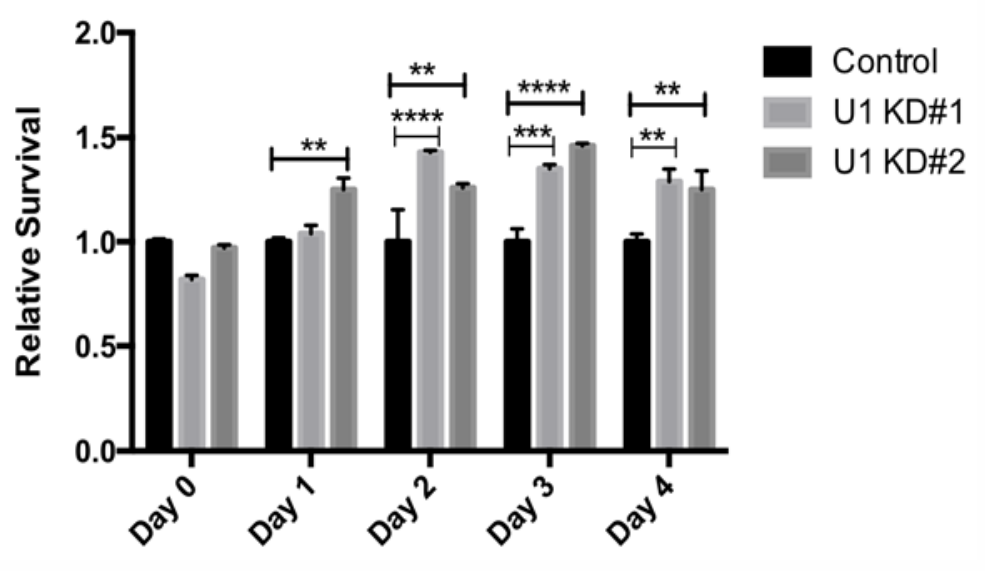

B

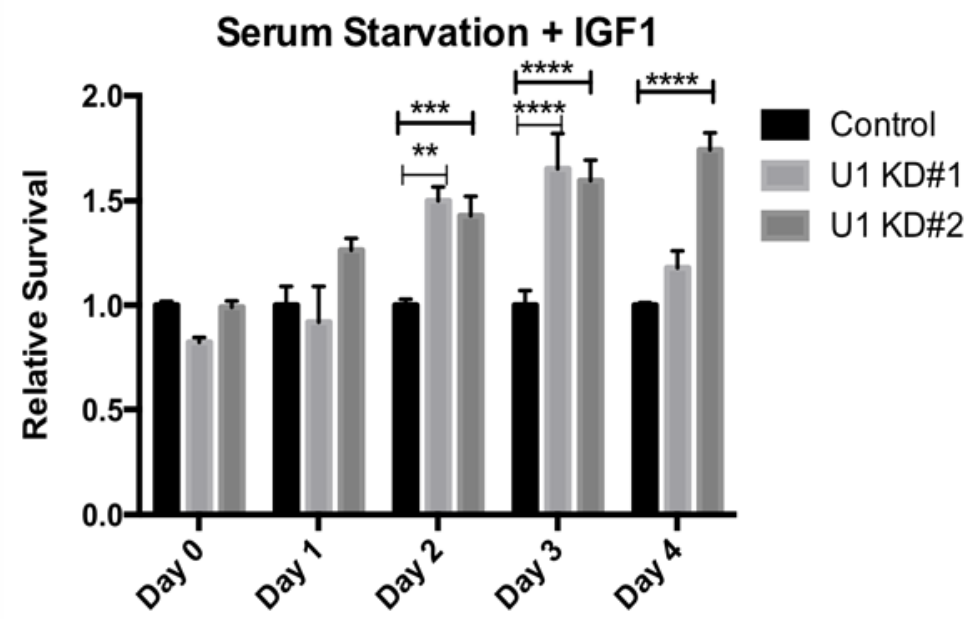

Figure 26: UBQLN1 deficient A549 cells have increased survival in serumfree conditions

A549 cells expressing stable shRNA against UBQLN1 (U1 KD\#1, U1 KD\#2, and control) were cultured in conditions of serum-free media and serum-free media supplemented with IGF1 for 4 days. Alamar Blue readings were recorded every 24 hours. Day 2 onwards, UBQLN1 deficient cells gradually begun to outsurvive control cells when cultured in serum-free media (A) and supplementing serumfree media with IGF1 (B) enhanced survival of these cells. Data are represented as mean \pm SEM from 2 independent experiments. ${ }^{*} p \leq 0.05,{ }^{* *} p \leq 0.01,{ }^{* * *} p \leq$ 0.001 , all relative to control in serum-free conditions. 


\section{Activation of IGF1 pathway in UBQLN1 deficient A549 cells increases their migration potential 3-fold}

We have previously published that lung adenocarcinoma cells (A549 and H358) that have loss of UBQLN1 are more migratory and invasive (Shah et al., 2015). Here, we investigated whether UBQLN1 deficient A549 cells migrate more upon stimulation of IGF pathway. In a transwell cell migration assay, cells were cultured in the top chamber in one of 3 conditions - serum-free media, serum-free media supplemented with IGF1 and serum-free media supplemented with IGF1 and Linsitinib, a small molecule inhibitor of IGF1R activity. Media supplemented with 10\% FBS was used as chemo-attractant in the bottom chamber. At the end of 24 hours, pictures of migrated cells were captured (Fig. 27A) and number of migrated cells were quantified by ImageJ software (Fig. 27B). UBQLN1 deficient cells are inherently more migratory compared to control as observed in serum-free conditions. When stimulated with IGF1, there was a 3 -fold increase in migration in UBQLN1 deficient cells compared to serum-free conditions $(p<0.01)$ whereas IGF1 stimulation did not significantly increase migration in control cells. Migration returned to baseline by Linsitinib in all cells and it appears that UBQLN1 deficient cells are more sensitive to inhibition than control. Based on these data, we conlude that activation of IGF1 pathway may prime UBQLN1 deficient cells to become more migratory. 
A

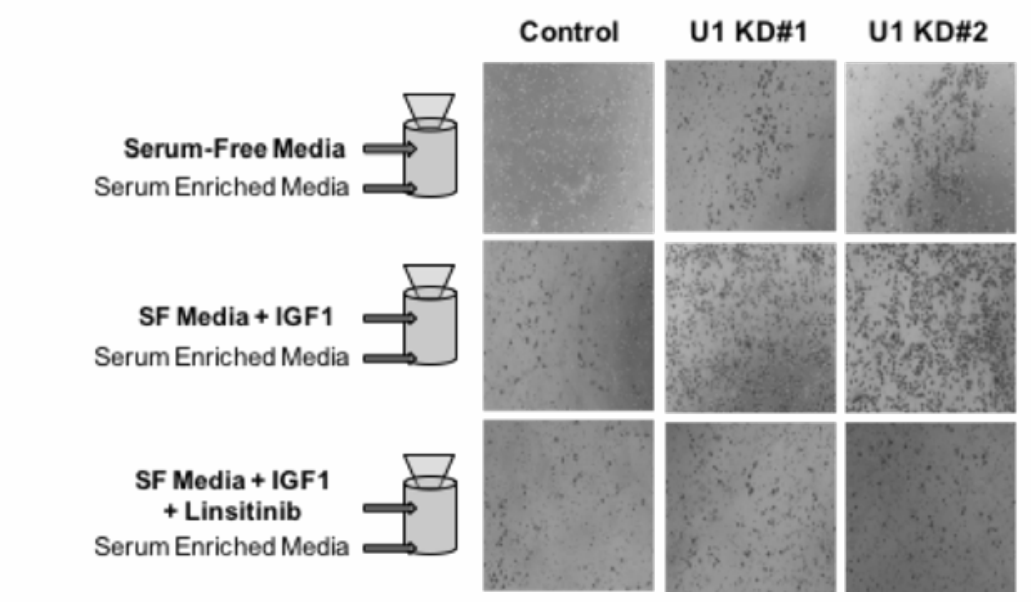

B

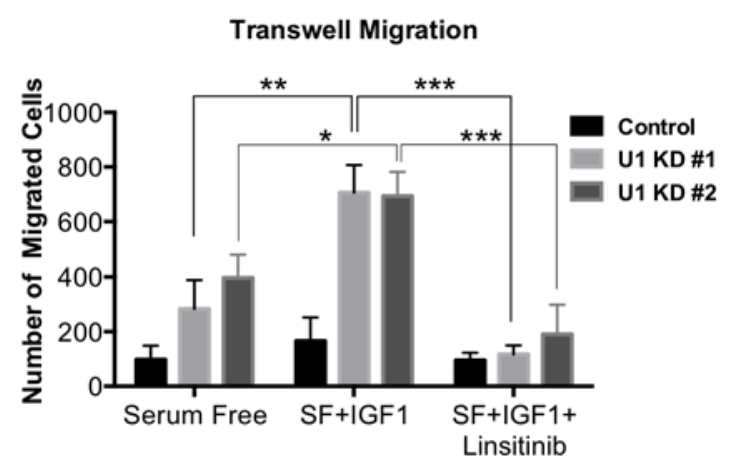

Figure 27: Activation of IGF1 pathway in UBQN1 deficient A549 cells increases their migration potential 3-fold

A549 cells expressing stable shRNA against UBQLN1 (U1 KD\#1, U1 KD\#2, and control) (Fig. 29E) were seeded in a transwell setup to assess cell migration in response to IGF1 stimulation. Cells were cultured in the top chamber in one of 3 conditions - serum-free media, serum-free media supplemented with IGF1 and serum-free media supplemented with Linsitinib, a small molecule inhibitor of IGF1R activity. Media supplemented with $10 \%$ FBS was used as chemo-attractant in the bottom chamber. At the end of 24 hours, cells were fixed and HEMA3 stained (A). Number of migrated cells were quantified by ImageJ software and analyzed by ANOVA (B). UBQLN1 deficient cells are inherently more migratory (3-4 times compared to control) as observed in serum-free conditions. When UBQLN1 deficient cells were stimulated with IGF1, there was a 3-fold increase in migration compared to control cells. Linsitinib blocked cell migration and brought it back to control cells' baseline levels. Data are represented as mean \pm SEM from three independent experiments. ${ }^{*} p \leq 0.05,{ }^{* *} p \leq 0.01,{ }^{* * *} p \leq 0.001$, all relative to control. 
STI-1/2 domains of UBQLN1 interact with Insulin receptor (INSR) and loss of UBQLN1 regulates expression of INSR and IGF2R post-stimulation with IGF1.

HEK293T cells were transfected domain deletion constructs of UBQLN1 followed by immunoprecipitation by FLAG antibody and probed for INSR. As with IGF1R, UBQLN1 interacts with INSR and we mapped this interaction to the STI-1/2 domains (Fig. 28). We examined whether UBQLN1 regulates expression of INSR as it does with IGF1R. A549 cells with stable expression of shRNA against UBQLN1 and control were cultured in 4 different conditions (Fig 29A): complete media, serum-free media for 12 hours and serumfree media (12 hours) supplemented with IGF1 (6 hours) and serum-free media (12 hours) supplemented with Linsitinib (1uM), an IGF1R activity inhibitor. Cells were incubated with Cycloheximide an hour before adding IGF1 to block de novo protein synthesis, which enabled assessment of steady state expression of INSR. Post-stimulation, cells were harvested, lysed and analyzed by Western Blot. The antibody used to detect phosphoIGF1R recognizes auto-phosphorylation site Tyr1135 on IGF1R and cross-reacts with Tyr1150 on INSR, which shows increased phosphorylation in UBQLN1 deficient cells. INSR expression in UBQLN1 knock down cells was notably decreased upon serumstarvation and stimulation with IGF1. Loss of UBQLN1 also caused loss of IGF2R upon ligand stimulation in A549 cells (Fig. 29B). Based on these data, we conclude that UBQLN1 interacts with INSR and regulates expression of INSR and IGF2R poststimulation with IGF1. Additionally, AKT, a critical regular of survival and apoptosis is activated (p-AKT) in A549 cells that have loss of UBQLN1 and more so when the IGF pathway is stimulated with exogenous ligand. Therefore, next we tested for survival of A549 cells in serum-free media and in presence of IGF1 with or without UBQLN1. 


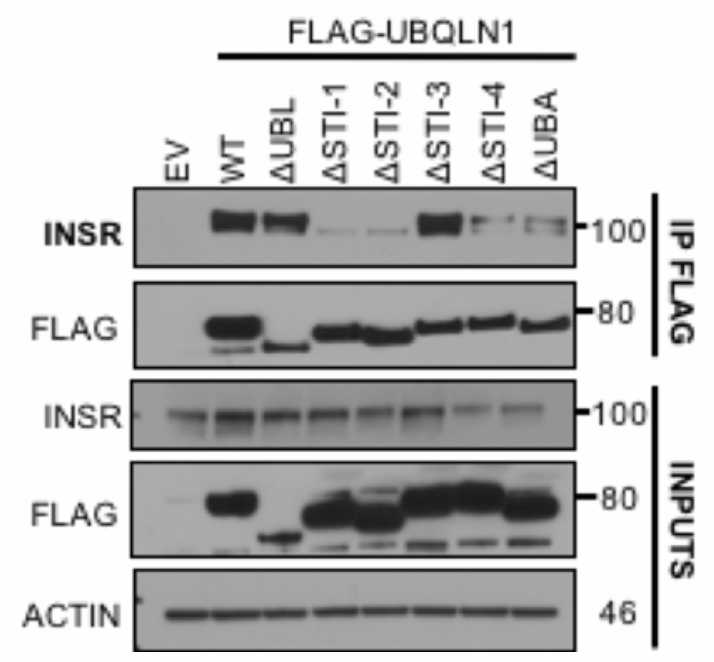

Figure 28: STI-1/2 domains of UBQLN1 interact with Insulin receptor (INSR)

HEK 293T cells were transfected with FLAG-tagged constructs of UBQLN1 followed by co-immunoprecipitation by FLAG antibody and Western Blot analysis. STI-1 and STI-2 domains of UBQLN1 are required for interaction with INSR. (Data are from one experiment). 
A

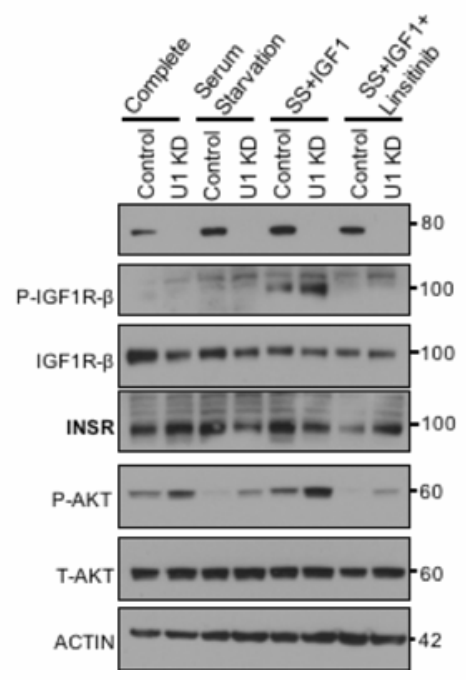

B

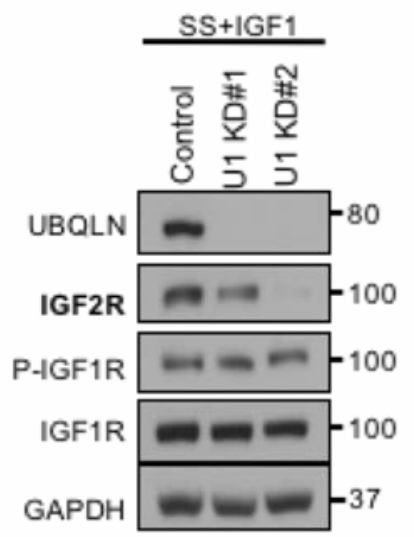

Figure 29: Loss of UBQLN1 regulates expression of INSR and IGF2R poststimulation with IGF1.

(A) Expression and activity of INSR were tested in A549 lung cancer cells (control and shRNA against UBQLN1: U1 KD\#1 and U1 KD\#2). Cells were serum starved (SS) for 12 hours, or supplemented with IGF1 (6 hours) or Linsitinib, a small molecule inhibitor of IGF1R activity. INSR expression was decreased in UBQLN1 deficient cells only in serum starved and IGF1-stimulated conditions. Additionally, UBQLN1 deficient cells showed increased basal expression of P-AKT which was enhanced by IGF1 stimulation, while TAKT levels remained constant across all conditions for both control and UBQLN1 deficient A549 cells. (B) Expression and activity of IGF2R were tested in the above cells. Cells were serum starved (SS) for 12 hours and supplemented with IGF as in D. Expression of IGF2R is decreased with loss of UBQLN1. (Data are from one experiment). 


\section{UBQLN1 deficient A549 cells have decreased autocrine ligand production compared to control}

We hypothesized that UBQLN1 deficient A549 cells may be responsible for autocrine production of its ligands (IGF1, IGF2, Insulin), which can consequently lead to activation of AKT and therefore survival and anti-apoptotic pathways. To investigate this, mRNA expression of IGF1 and IGF2 were tested in these cells cultured in complete media and serum-free conditions (72 hours). Contrary to our hypothesis, IGF1 expression was significantly lower in UBQLN1 deficient cells in both conditions (Fig. 30A,B). IGF2 expression was unchanged between control and UBQLN1 deficient cells in complete media (Fig. 30C,D). Although not statistically significant, when serum starved, production of IGF2 seemed to be decreasing in UBQLN1 knock down cells compared to control. Based on these data, we conclude that survival advantage conferred to cells upon UBQLN1 knock down may not be due to autocrine ligand production but mediated through other intracellular survival pathways. 
A

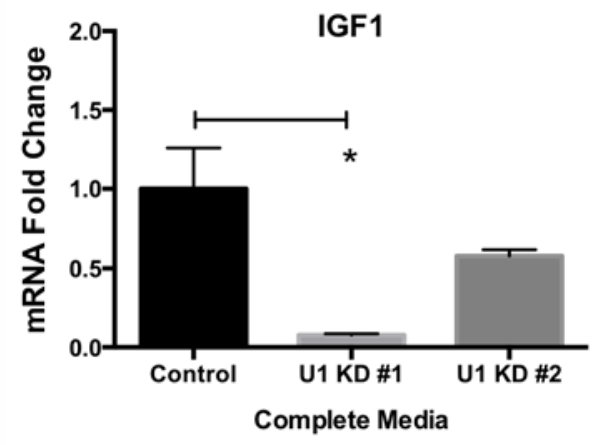

C

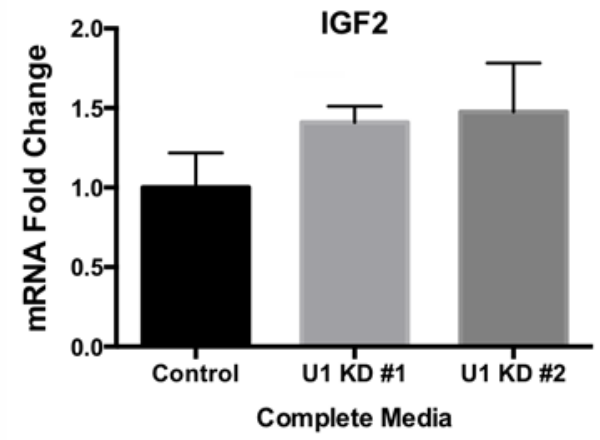

B

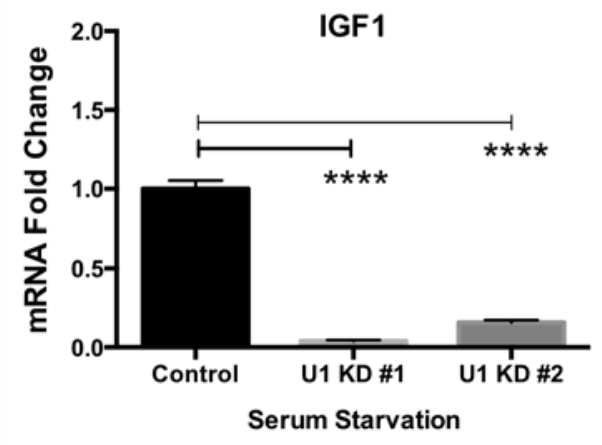

D

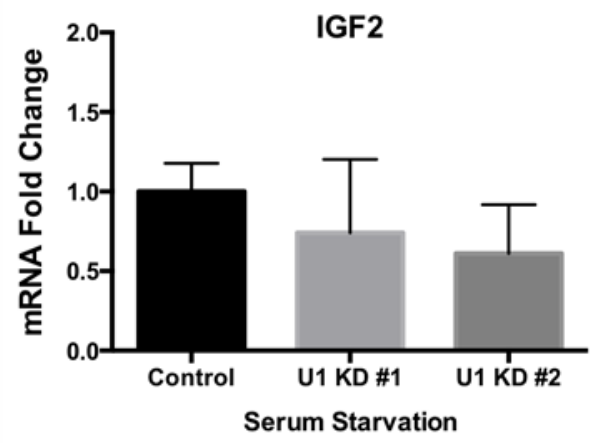

Figure 30: UBQLN1 deficient A549 cells have decreased autocrine ligand production compared to control

A549 cells expressing stable shRNA against UBQLN1 (U1 KD\#1, U1 KD\#2 and control) were cultured in conditions of complete media or serum-free media for 72 hours and real time analysis of IGF1 and IGF2 mRNA were performed. UBQLN1 deficient A549 cells showed significantly decreased production of IGF1 compared to control when these cells were cultured in both, complete $(\mathbf{A})(p<0.05)$ and serum-free media (B) $(p<0.001)$. Production of IGF2 was unchanged between these cells in complete media (C) but showed a decreasing trend in UBQLN1 deficient cells upon serum starvation (D). These data were not statistically significant. (Data are averaged from 3 individual experiments). 
UBQLN1 does not stably associate with these markers of endocytic trafficking: GRB10, EEA1, RAB7, LAMP1, Caveolin1

Loss of UBQLN1 altered Iysosomal trafficking of IGF1R and Percoll density gradient fractionation showed that there may be a slight shift of IGF1R toards higher density gradients like the lysosomes, we investigated whether UBQLN1 interacts with IGF1R as it is internalized and proceeds through the endocytic pathway and possibly alters its trafficking (Fig. 31). We simply tested UBQLN1's interaction with a few known markers on endocytosis. FLAG-UBQLN $1^{\mathrm{WT}}$, UBQLN $1^{112 \mathrm{X}}$ and UBQLN1 $1^{\triangle \mathrm{UBA}}$ were overexpressed in cells followed by Immunoprecipitation pull down with FLAG antibody and probed for the respective proteins. We did not detect an interaction for any of these markers. However, based on these data, we cannot exclude UBQLN1's role in trafficking of IGF1R. 


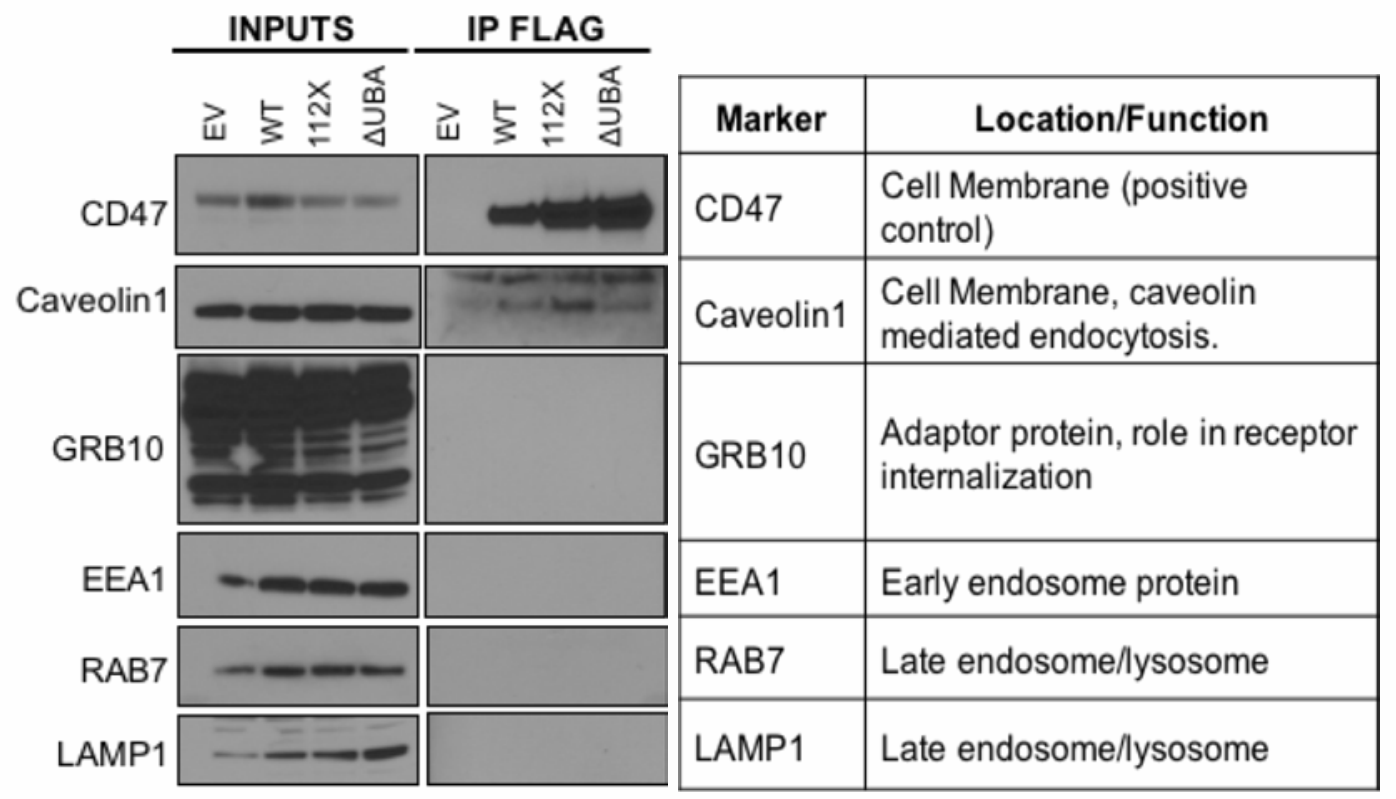

Figure 31: UBQLN1 does not stably associate with these markers of endocytic trafficking: GRB10, EEA1, RAB7, LAMP1, Caveolin1

HEK 293T cells were transfected with FLAG-UBQLN1 ${ }^{\text {WT }}$, UBQLN $1^{112 X}$ and UBQLN1 $1^{\triangle \mathrm{UBA}}$ followed by immunoprecipitation by FLAG antibody and Western Blot analysis. Interaction was tested for the markers of the endocytic pathway as shown above in the table. CD47 or Integrin Associated Protein (IAP) was used as a positive control, as a known interacting partner of UBQLN1. UBQLN1 did not interact with these proteins tested. However, a faint interaction is detected for Caveolin1, a marker of the caveolin-mediated endocytosis. (Data are from one experiment). 
Combined loss of UBQLN1 and PTP1B has a synergistic effect on activation of kinase domain of IGF1R

Since UBQLN1 deficient cells have an increased ratio of active:total IGF receptors, we investigated whether this effect was exclusive of action of phosphatases for IGF1R. H358 lung adenocarcinoma cells were transfected with siRNA for UBQLN1 and PTP1B, a known phosphatase of IGF1R. Cells were cultured in complete media and 48 hours posttransfection, Western Blot analysis were performed and probed for auto-phosphorylation and kinase activation sites of IGF1R by specific antibodies as shown (Fig. 32). Loss of UBQLN1 showed slight increase in auto-phosphorylation of IGF1R compared to control. Loss of PTP1B did not show more auto-phosphorylation compared to control. Combined effect of loss of both, showed no additional increases in auto-phosphorylation of IGF1R. However, combined loss of UBQLN1 and PTP1B have a synergistic effect on activation of kinase domain of IGF1R. 


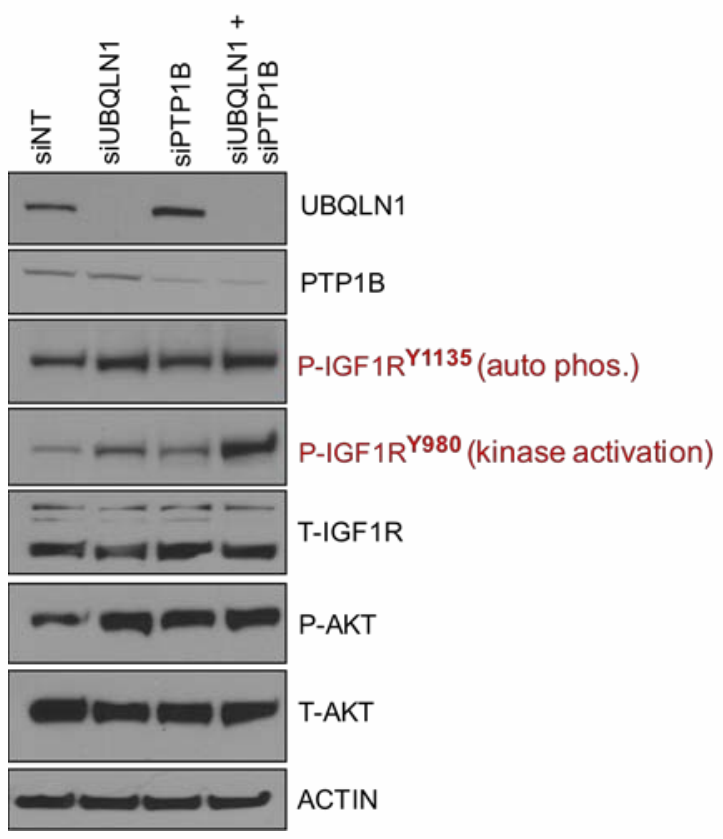

Figure 32: Combined loss of UBQLN1 and PTP1B has a synergistic effect on activation of kinase domain of IGF1R

H358 lung adenocarcinoma cells were transfected with siRNA for UBQLN1 and PTP1B. Cells were cultured in complete media and 48 hours post-transfection, Western Blot analysis were performed and probed for auto-phosphorylation and kinase activation sites of IGF1R by specific antibodies as shown. Loss of UBQLN1 and combined loss of UBQLN1 and PTP1B show a small increase in autophosphorylation of IGF1R. However, there is a synergistic effect of combined loss of both proteins on activation of kinase domain of IGF1R. (Data are from one experiment). 
UBQLN1 and UBQLN2 interact with Epidermal Growth Factor Receptor (EGFR)

Since UBQLN1 interacts with and reguates IGF receptor tyrosine kinases, we examined whether UBQLN also interacts with other receptor tyrsoine kinases like EGFR. FLAGUBQLN1 and FLAG-UBQLN2 were overexpressed in HEK293T cells followed by immunoprecipitation by FLAG antibody. Both, UBQLN1 and UBQLN2 interact with EGFR (Fig. 33). 


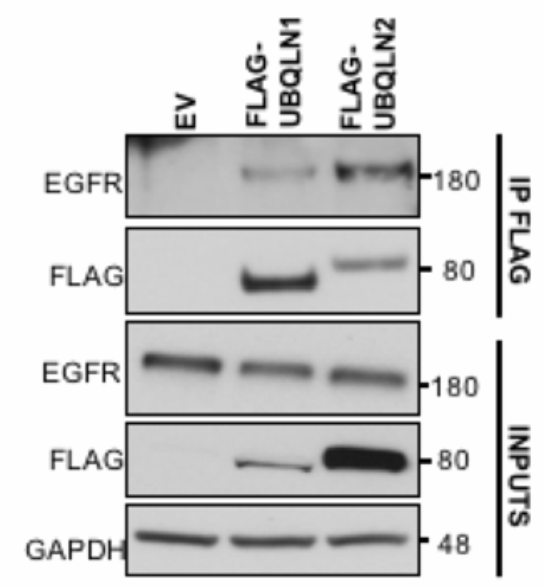

Figure 33: UBQLN1 and UBQLN2 interact with EGFR

HEK293T cells were transfected with empty vector (EV) or FLAG-UBQLN1 or FLAGUBQLN2. 48 hours post transfections, cells were lysed followed by immunoprecipitation by FLAG antibody and probed for EGFR by Western Blot analysis. Both, UBQLN1 and UBQLN2 interact with EGFR. (Experiment was performed twice). 
UBQLN1 regulates EGFR expression in K-RAS mutant A549 cells but not in EGFR mutant $\mathrm{H} 1650$ cells

We investigated whether UBQLN1 can universally regulate EGFR expression and therefore tested for effects of loss of UBQLN1 in k-ras mutant A549 (Fig. 34A) and EGFR mutant H1650 (Fig. 34B) lung adenocarcinoma cells. These cells were transfected with siRNA for UBQLN1. Cells were serum starved (SS) for 12 hours, or supplemented with EGF (50ng/ml for 1 hour) or Erlotinib (50nM), a small molecule inhibitor of EGFR kinase domain. A549 cells showed increased phosphorylated EGFR levels in UBQLN1 deficient cells and decreased total EGFR expression was in UBQLN1 deficient cells only in EGFstimulated condition. These data are similar to UBQLN1's regulation of IGF1R. However, H1650 cells did not show a similar effect. Although, phosphorylated EGFR expression was increased in UBQLN1 deficient cells in complete media, this effect was very slight and not accompanied with decrease in but total receptor expression as seen in A549 cells. Based on these data, we conclude that UBQLN1 regulates wild type EGFR but not mutant EGFR (E746-750Adel). A549 cells show increased basal phosphorylation of AKT in UBQLN1 deficient cells in all conditions tested as also seen in Fig. 29A. However, basal p-AKT expression completely disappeared upon inhibition of EGFR activity but not with inhibition of IGF1R activity. This implies that between these 2 receptor pathways, stimulation of EGFR has a dominant effect on AKT activation (phosphorylation of Ser 473) and may be partly responsible for increased basal expression of p-AKT in UBQLN1 deficient cells. 
A

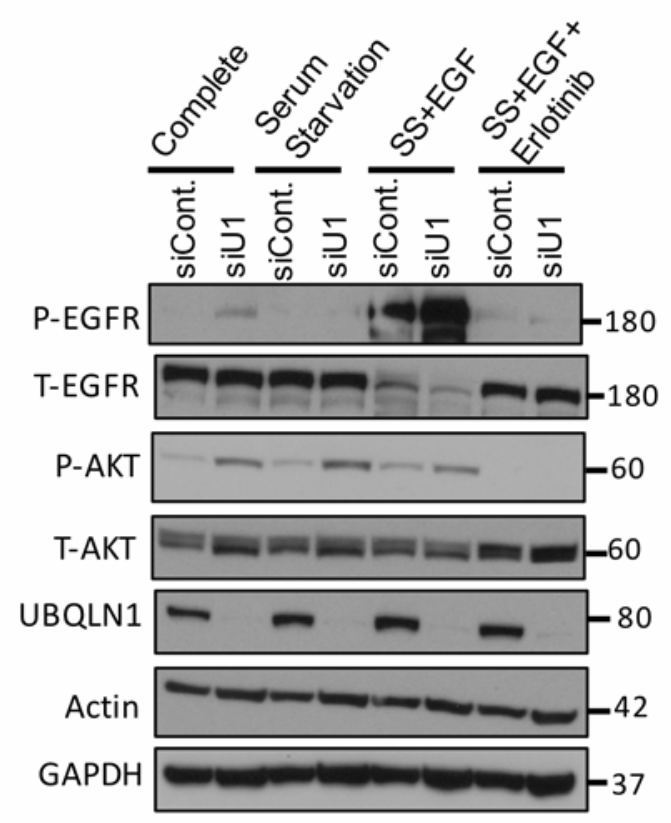

A549
B

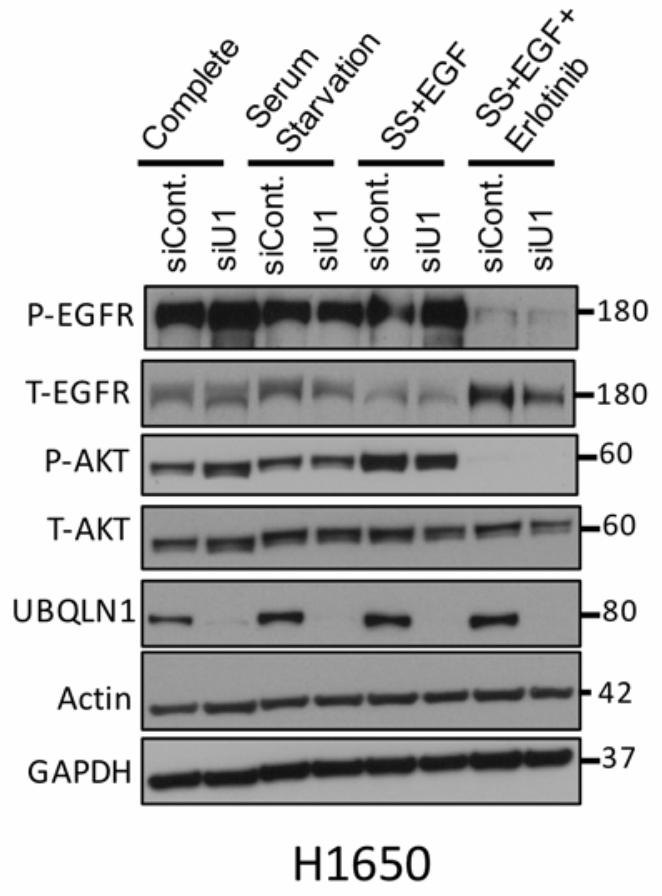

Figure 34: UBQLN1 regulates EGFR expression in K-RAS mutant A549 cells but not in EGFR mutant $\mathrm{H} 1650$ cells

A549 and H1650 lung adenocarcinoma cells were transfected with siRNA for UBQLN1. Cells were serum starved (SS) for 12 hours, or supplemented with EGF (50ng/ml, 1 hour) or Erlotinib (50nM), a small molecule inhibitor of EGFR kinase domain. (A) A549 cells showed increased phosphorylated EGFR levels in UBQLN1 deficient cells. Total EGFR expression was decreased in UBQLN1 deficient cells only in EGF-stimulated condition. (B) Phosphorylated EGFR expression was increased in UBQLN1 deficient $\mathrm{H} 1650$ cells in complete media, but total receptor expression didn't change. (Experiment was performed twice). 


\section{EGF-dose dependent regulation of EGFR by UBQLN1}

We observed that loss of UBQLN1 accelerates loss of EGFR post-stimulation with ligand, as seen with IGF1R. Here we tested loss in expression of EGFR with 2 different doses of EGF in A549 cells (Fig. 35). Cells were serum starved (SS) for 12 hours and supplemented with EGF (1 hour) with 2 different doses: 10ng/ml (Fig. 35A) and 100ng/ml (Fig. 35B). Overall, phosphorylated EGFR expression was higher when cells were stimulated with 100ng/ml EGF. For both ligand concentrations, phosphorylated EGFR expression was higher in UBQLN1 deficient cells. For both ligand concentrations, EGFR expression was decreased faster in UBQLN1 deficient cells and more pronounced with higher dose of ligand. Based on these data, we conclude that UBQLN1 may regulate EGFR in a EGFdose dependent manner. 
A

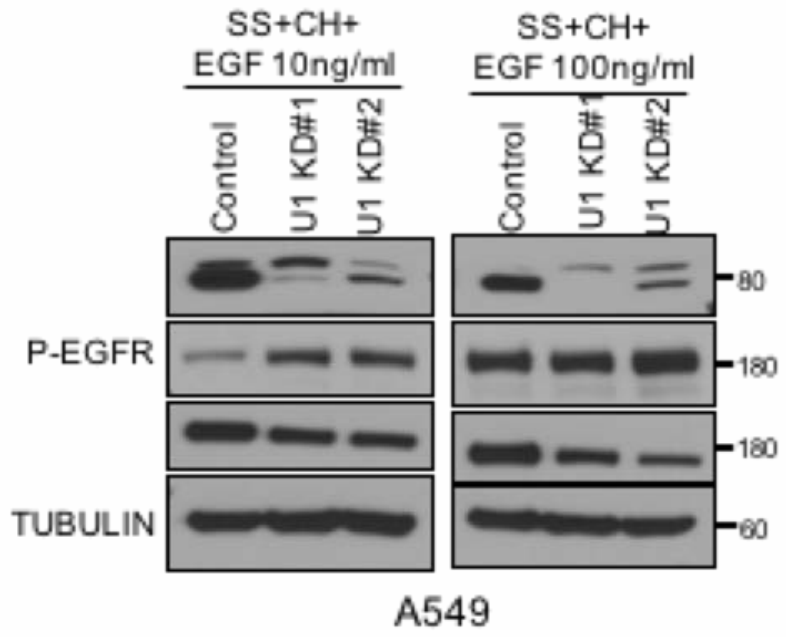

Figure 35: EGF-dose dependent regulation of EGFR by UBQLN1

A549 were transfected with siRNA for UBQLN1. Cells were serum starved (SS) for 12 hours and supplemented with EGF (1 hour) with 2 different doses: 10ng/ml (A) and $100 \mathrm{ng} / \mathrm{ml}$ (B). Overall, phosphorylated EGFR expression was higher in UBQLN1 deficient cells for both ligand doses. Total EGFR expression decreased with receptor activation by ligand but its loss is faster in UBQLN1 deficient cells. 


\section{DISCUSSION}

Role of UBQLN proteins is slowly emerging in the field of cancer. Recently, it was reported that increased UBQLN2 expression within osteosarcoma cells enhances tumor progression in patients. UBQLN2 expression in urine was also proposed to be used as a biomarker to diagnose, stage and grade urothelial cancer (Shimada et al., 2016). Our lab has published that UBQLN1 is lost and under-expressed in approximately fifty percent of non-small cell lung cancers (Shah et al., 2015). Cells that have loss of UBQLN1 adopt a more epithelial-to-mesenchymal (EMT) phenotype and are more and migratory and invasive (Shah et al., 2015). In this manuscript, we determined the role of UBQLN1 in regulation of IGF1R, a receptor tyrosine kinase overexpressed in lung cancer.

We have previously determined that fate of substrates UBQLN1 associates with is interaction domain specific. Transmembrane proteins like BCLb, ESYT2, IGF1R (Fig. 19), INSR (Fig. 29) interact with UBQLN1 through its STI-1 and STI-2 domains and are stabilized as a result of this interaction. UBQLN1 does not alter stability of substrates involved in proteasomal machinery like BAG6 and PSMD4 that associate through its UBL domain (Kurlawala et al., 2017). UBQLN1's interaction with IGF1R is unusual because unlike other regulators, this interaction is not dependent on phosphorylation of IGF1R. UBQLN1 interacts with all forms of IGF1R - non-processed immature form (pro-IGF1R) ,phosphorylated form and non-phosphorylated form (Fig. 19). It appears that UBQLN1 is recruited to IGF1R from the time of its synthesis and remains bound to it during trafficking. Known binding partners of IGF1R like IRS (L. M. Wang et al., 1993), Shc (Wills \& Jones, 2012), Grb2 , Grb10 (Dufresne \& Smith, 2005; Langlais et al., 2004; Mori, Giovannone, \& Smith, 2005; Ramos, Langlais, Hu, Dong, \& Liu, 2006; Stein, Gustafson, \& Hubbard, 2001) are recruited to IGF1R only when the receptor is stimulated by ligand, autophosphorylated, and have undergone structural conformation to accommodate binding 
IRS and Shc proteins are one of the first to bind to IGF1R which allows subsequent recruitment of more molecules in turn stimulating other signaling pathways. GRB10 (direct recruitment) and GIGYF2 (recruitment through GRB10) bind to IGF1R and have been identified as negative regulators of IGF1R phosphorylation (Giovannone et al., 2009). We found that UBQLN1 is a positive regulator of total-IGF1R expression. Loss of UBQLN1 causes accelerated loss of IGF1R and overexpression of UBQLN1 stabilizes it (Kurlawala et al., 2017).

Role of UBQLN1 has been studied in regulation of cell surface receptors like GABA $_{A}$ receptors (Saliba et al., 2008), Presenilins (Mah et al., 2000; Massey et al., 2004; Massey et al., 2005), GPCR's (N'Diaye et al., 2008) and nicotinic acetylcholine receptors (Ficklin et al., 2005) and others. Regulation of IGF1R by UBQLN1 is similar to its regulation of $\mathrm{GABA}_{\mathrm{A}}$ receptors which has been studied in detail (Bedford et al., 2001; Saliba et al., 2008). UBQLN1 interacts with $\mathrm{GABA}_{A}$ receptors through its UBA domain. Loss of interaction led to decrease in receptor numbers, without an effect on rate of internalization or endocytosis. Similarly, UBQLN1 stabilizes full length Presinilins and prevents its fragmentation within the gamma secretase complex embedded in the plasma membrane (Massey et al., 2005). In A549 lung cancer cells, loss of UBQLN1 causes accelerated loss of IGF1R without gross changes in trafficking or phosphorylation activity. We have a few theories that may possibly explain this phenomena. Loss of UBQLN1 causes decreased mRNA expression of IGF1R. When new protein synthesis is blocked by Cycloheximide, loss of UBQLN1 can lead to faster loss of the relatively smaller existing pool of receptors. We have not determined whether UBQLN1 has a direct or indirect effect on gene transcription of IGF1R but based on our immunofluorescence data in variety of cell types (HEK 293T, HeLa, A549, H358 and HPL1D, Fig. 2, 19, 20), we have not detected nuclear presence of UBQLN1 thus far. Another reason for decreased transcription of IGF1R could be the negative feedback loop caused by a relatively active IGF pathway seen in UBQLN1 
deficient cells leading to suppression of signal for new receptor synthesis. Once translated, multi-subunit proteins like IGF1R and $\mathrm{GABA}_{\mathrm{A}}$ receptors are assembled and processed in the Endoplasmic Reticulum and Golgi before delivery and insertion to designated organelles. However, the process of assembly is inefficient and only $25 \%$ of translated proteins are assembled into mature forms. Overexpression of UBQLN1 caused a $\sim 30 \%$ increase in Endo $H$ sensitive $\mathrm{GABA}_{\mathrm{A}}$ receptors in the ER (Saliba et al., 2008). UBQLN1 interacts with the higher molecular weight $(\sim 135 \mathrm{kD})$, pro-form of IGF1R and therefore maybe involved in assembly of IGF1R in the ER. Additionally, transport of these receptors to the cell surface takes about 4-6 hours. It is yet to be investigated how long after loss of interaction between UBQLN1 and IGF1R, does IGF1R expression begin to decrease. If the decrease in expression occurs within a matter of minutes after loss of interaction, it would indicate a stability/degradation based mechanism. However, if it takes hours, it would solidify UBQLN1's role in assembly in the ER of IGF1R other transmembrane proteins like INSR, BCLb, ESYT2, OMP25, and so on.

UBQLN1 also interacts with Insulin receptor and loss of UBQLN1 regulates INSR expression when A549 cells are serum starved and activated by IGF1 (Fig. 20, 29).

Despite having decreased total receptor levels in UBQLN1 deficient cells, the ratio of active to inactive IGF1R was at least 2 times higher (Fig. 20) and combined loss of UBQLN1 and PTP1B caused a synergistic increase in phosphorylation of the kinase domain of IGF1R (Fig. 32). This may indicate that normal dephosphorylation of IGF1R may be delayed in absence of UBQLN1. PTP1B and SHP2 are known phosphatases that negatively regulate IGF1R phosphorylation in a ligand dependent manner (Buckley, Cheng, Kiely, Tremblay, \& O'Connor, 2002). Phosphatases are recruited to the plasma membrane via adaptor proteins like SHPS2. It is known that Integrin ${ }_{\alpha \vee \beta 3}$ acts via SHP2 to dephosphorylate IGF1R (Rocchi, Tartare-Deckert, Sawka-Verhelle, Gamha, \& van Obberghen, 1996). It is a plausible hypothesis that UBQLN1 recruits phosphatases to the 
receptor for normal dephosphorylation directly or through participation in a multi-protein complex involving Integrins and other cell surface proteins that help recruit phosphatases to IGF1R to enable normal dephosphorylation.

One of UBQLN1's primary functions is in protein quality control and depending on type of substrate either facilitates substrate degradation or stabilization. We have previously shown that transmembrane proteins like BCLb, ESYT2 and IGF1R are stabilized by interaction with UBQLN1 and its UBA domain protects from MG132 (proteasomal inhibitor) mediated degradation in HEK 293T cells. Here, we investigated in A549 lung cancer cells whether UBQLN1 plays a role in normal turnover of IGF1R. IGF1R is degraded via both lysosomal and proteasomal pathways. Therefore, we blocked both pathways individually with inhibitors to determine role of UBQLN1 in degradation of IGF1R. IGF1R recycling is prevented upon loss of UBQLN1 as the same effect is seen with Monensin (Fig. 23) whereas blocking the proteasome did not seem to have a profound effect (Fig. 24). Additionally, IGF1R was detected to be slightly shifted towards higher density compartments (ex. Lysosomes) upon loss on UBQLN1 (Fig. 25). Taken together, we conclude that UBQLN1 plays a role in lysosomal turnover of IGF1R.

We then studied the biological relevance of UBQLN1's association with IGF1R in context of cancer progression. We determined that UBQLN1 deficient A549 cells showed improved survival when serum starved (Fig. 26). This phenomena may be at least partially attributable to higher basal levels of active AKT protein in UBQLN1 deficient cells (Fig. 29). Stimulation of the IGF pathway corresponded with an enhanced survival advantage (Fig. 26), possibly due to additional activation of AKT, a key central molecule activated downstream of IGF1R stimulation that via multiple mechanisms evades apoptosis and 
promotes survival for example directly inactivating pro-apoptotic Bad, inactivating Caspase 9 (Datta et al., 1997), increasing expression of anti-apoptotic NFKB which then downregulates p53 (Jeong, Pise-Masison, Radonovich, Park, \& Brady, 2005).

We have previously shown that lung adenocarcinoma cells that have loss of UBQLN1 adopt a more Epithelial-to-Mesenchymal Transition (EMT) like phenotype and are more migratory (Shah et al., 2015). Here, we found that stimulating UBQN1 deficient A549 cells with IGF1 increased their migratory potential 3-fold (Fig. 27). As UBQLN1 interacts with several plasma membrane proteins like IGF1R, INSR, IAP, it is worth testing whether UBQLN1 participates in a multi-protein complex with these receptors at the plasma membrane such that loss of UBQLN1 facilitates increased ECM-cell communication promoting migration. The IGF pathway is known to promote cell migration in cancer cells through multiple pathways. It can cause disassembly of adherens junctions and redistribution of movement fibers promoting motility (Cox et al., 2015). In communication with the microenvironment, IGF1R signaling can induce expression of proteases like cathepsin D (F. Wang, Duan, Chirgwin, \& Safe, 2000), matrix metalloproteinases (Yoon \& Hurta, 2001; D. Zhang \& Brodt, 2003) and urokinase plasminogen activator (Dunn, Torres, Oh, Cykert, \& Barrett, 2001), promoting disintegration of basement membranes to facilitate cell migration. These proteases can also bind and cleave IGFBP3, which can release bound IGF1 for further stimulation (Cohen et al., 1992). Integrin activation from the extracellular matrix can also regulate IGF1R signaling to promote migration (Clemmons \& Maile, 2003).

UBQLN1 interacts with receptor tyrosine kinases outside of the IGF pathway, such as the Epidermal Growth Factor Receptor, which is also mutated and overexpressed in a variety of cancers. We demonstrated that UBQLN1 and UBQLN2 interact with EGFR. For the two doses of EGF stimulation we tested $(10 \mathrm{ng} / \mathrm{ml}$ and $100 \mathrm{ng} / \mathrm{ml})$ UBQLN1 regulates activity of EGFR ${ }^{\mathrm{WT}}$ in $\mathrm{A} 549$ cells in an EGF-dose dependent manner. 
In conclusion, the IGF1 pathway axis has been a major target of research for chemotherapy. However, drugs that seem effective in in vitro, in vivo, and in early phase trials fail to show overall clinical benefit. The IGF pathway is complicated with presence of different receptor types including hybridization between receptors, multiple ligands, autocrine and paracrine stimulation, extensive influence of extracellular matrix signals, and compensatory signaling via other growth factor receptors. Additionally, intracellular IGF1R activity does not always correlate with its cell surface expression. We have also observed that IGF1R expression varies with cell confluence in vitro (data not shown). All these factors make it challenging to predict response to therapy thus highlighting the role of defining better biomarker profiles for choosing patients that will benefit from anti-IGF1R therapy. Therefore, it is essential to study cellular and extracellular factors that regulate activity of IGF1R directly or indirectly. Our data indicate that UBQLN1 expression is one such candidate. Future studies investigating role of UBQLN1 in IGF pathway mediated tumorigenesis, metastasis, response and resistance to therapy are warranted. Refer to Fig. 36 for model of regulation of IGF1R by UBQLN1. 
A

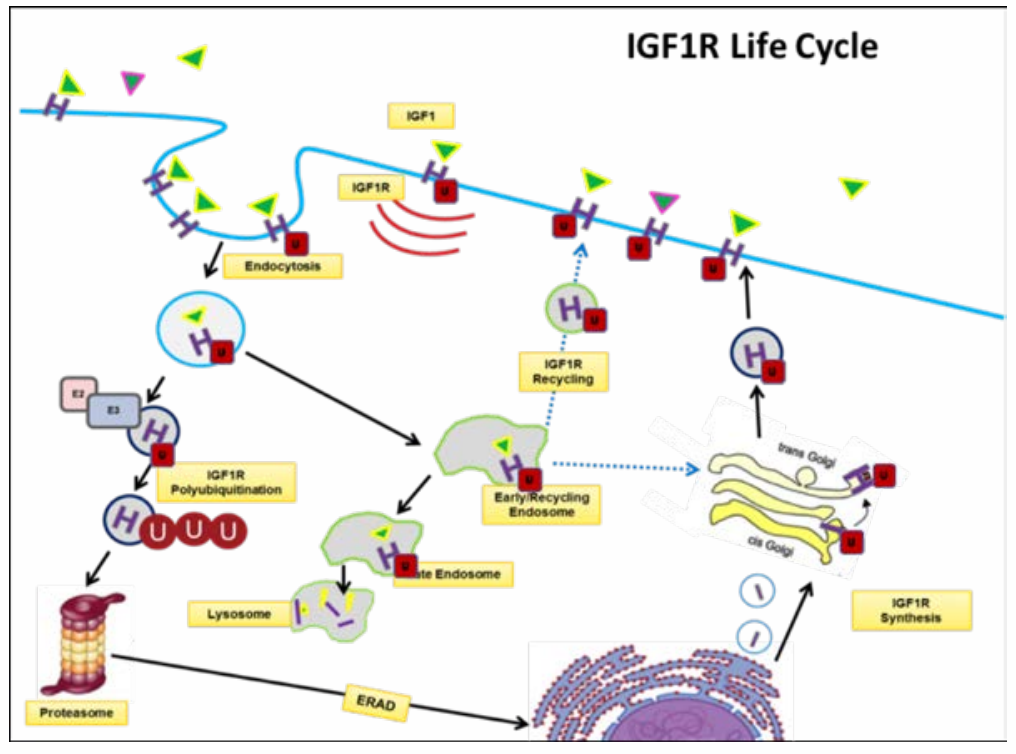

B

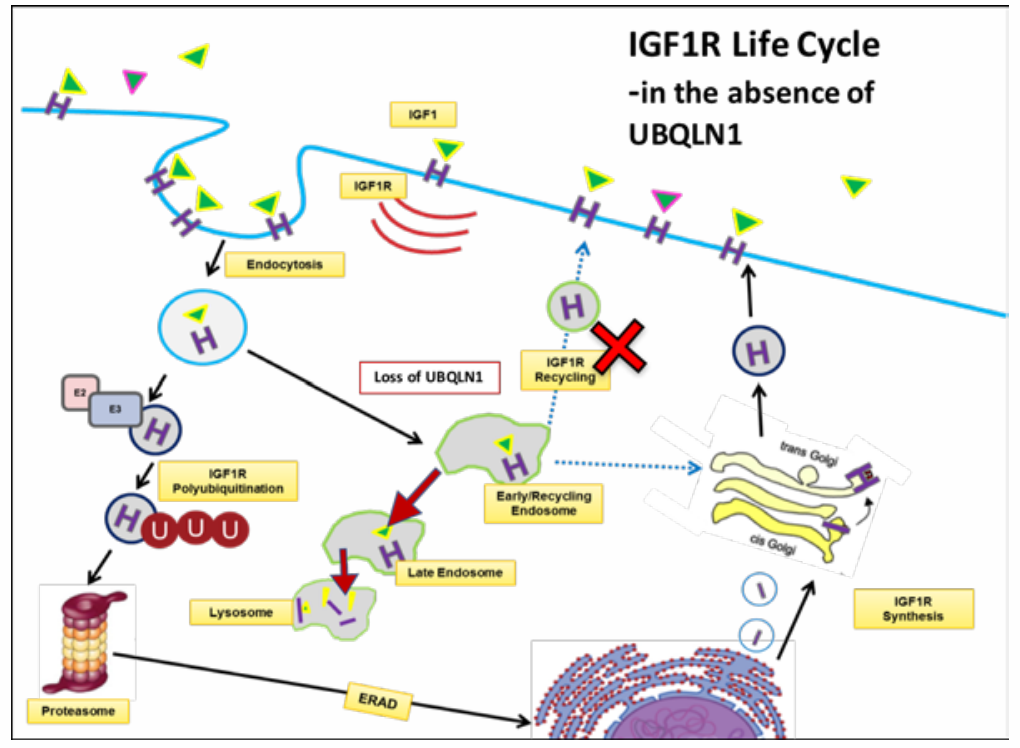

Figure 36: Model of regulation of IGF1R by UBQLN1: When UBQLN1 is present, it interacts with IGF1R from the time of its synthesis and assembly in the ER to processing in the Golgi to transport to the cell surface and continues to be recruited to the receptor upon ligand stimulation and internalization. Presence of UBQLN1 (A) allows normal recycling and turnover of the receptor. Absence of UBQLN1 (B) prevents normal recycling of the receptor, causes its accumulation and leads to eventual degradation by lysosomal and proteasomal pathways. 
CHAPTER 5

\section{OVERALL SUMMARY}

\section{Overall Goals and Specific Aims}

The Ubiquilin family proteins are implicated in a variety of cellular processes like autophagy, ERAD, receptor trafficking, proteasomal degradation, substrate stabilization and diseases like neurodegeneration and cancer. The overall goal of this dissertation was to identify a mechanism of action for UBQLN1. To do so, we developed the following aims:

\section{Specific Aims}

1) To investigate biochemical functions of UBQLN1

a) Identify domains of UBQLN1 responsible for interaction with multiple substrates

b) Determine role of UBQLN1 on substrate stability

2) To investigate role of UBQLN1 in regulation of receptor tyrosine kinases

a) Determine role of UBQLN1 in trafficking and turnover of IGF1R

b) Determine biological consequences of UBQLN1 loss and dysregulation of IGF pathway in lung adenocarcinoma cells.

c) Determine role of UBQLN1 in regulation of INSR and EGFR 


\section{Major Findings of this Dissertation}

We demonstrated that Ubiquilin1 regulates transmembrane proteins like $\mathrm{BClb}$, an anti-apoptotic mitochondrial membrane protein, insulin-like growth factor 1 receptor (IGF1R), a cell surface receptor tyrosine kinase, Extended Synaptotagmin 2 (ESYT2), a calcium sensing protein localized at ER-plasma membrane contact sites. The STI domains of UBQLN1 are essential for interaction with these substrates while the UBA domain is required to protect them from degradation.

\section{BCLb}

Using BCLb as a model substrate, we characterized UBQLN1-substrate interaction. We identified the first two STI domains of UBQLN1 as critical for binding to BCLb. Interaction of UBQLN1 with BCLb is independent of ubiquitination of BCLb, but interaction with ubiquitin via UBA domain is required for stabilization of BCLb. Similarly, we showed that UBQLN1 interacts with IGF1R and ESYT2 through the STI domains and stabilizes these proteins through its UBA domain. Interactions that are not dependent on STI domains, for example, UBL mediated interaction with PSMD4 and BAG6, do not appear to be stabilized by UBQLN1. We conclude that fate of substrates that UBQLN1 associates with, is interaction domain specific.

\section{ESYT2}

There are three Extended Synaptotagmin proteins (ESYT1/2/3). ESYTs have an $\mathrm{N}$-terminal ER-membrane binding domain, a mitochondrial-lipid-binding protein domain (SMP), and multiple calcium sensing C2 domains (Min, Chang, \& Südhof, 2007). Proteins like ESYTs connect the vast ER network to different compartments in the cell like plasma membrane and mitochondria and facilitate exchange of molecules and ions and regulate cell signaling between these compartments. ESYT2/3 are not integral membrane proteins like ESYT1, but are inserted into the ER membrane through its $\mathrm{N}$-terminal transmembrane domain and with the plasma membrane through its three C2 domains. Recently, it was 
shown that ESYT2 interacts with activated Fibroblast Growth Factor Receptor (FGFR) (Tremblay et al., 2015). Subsequently, ESYT2 interacts with early endosome marker EEA1 during the internalization phase of FGFR upon activation with FGF. Additionally, ESYT2 interacts with AP2, another protein involved in clathrin-mediated endocytosis (Jean et al., 2010). Our data demonstrate that UBQLN1 strongly interacts with ESYT1 and ESYT2 but not with ESYT3 (Immunoprecipitation/Mass Spectrometry). We confirmed that STI domains of UBQLN1 are critical in binding to ESYT2 (Immunoprecipitation/Western Blot) and the UBA domain protects it from MG132 induced degradation (Kurlawala et al., 2017).

\section{Receptor Tyrosine Kinases (IGF1R, IGF2R, INSR, EGFR)}

Our IP/MS and IP/WB data indicate that Ubiquilin1 interacts with IGF1R, IGF2R and INSR. Like with BCLb, the STI-1 and STI-2 domains are responsible for interaction with IGF1R and the UBA domain is critical in protecting it from MG132 (proteasomal inhibitor) induced degradation.

Next, we chose to study effects of loss of UBQLN1 on IGF1R in a lung adenocarcinoma cell line as IGF receptors are overexpressed in lung cancers. We demonstrate here that UBQLN1 regulates expression and activity of IGF1R. Following loss of UBQLN1 in lung adenocarcinoma cells, there is accelerated loss of IGF1R, partially rescued by blocking the proteasome. Despite decreased levels of total receptors, the ratio of active:total receptors is higher in cells that lack UBQLN1. UBQLN1 also regulates INSR and IGF2R post-stimulation with ligand. We conclude that UBQLN1 is essential for normal regulation of IGF1R. UBQLN1 deficient cells demonstrate increased survival when serum starved and stimulation of IGF pathway in these cells increased their migratory potential by 3-fold.

Additionally, we found that UBQLN1 and UBQLN2 interact with EGFR. UBQLN1 regulates expression of EGFR in a EGF-dose dependent fashion. 


\section{Substrate Stabilization Model for Ubiquilin1}

We demonstrated that STI and UBA mediated interactions of Ubiquilin1 result in proteostasis of three transmembrane proteins such that primary association of UBQLN1 with substrate occurs through the STI domains and a secondary association through its UBA domain leads to stabilization of the substrate. Additionally, we showed that the UBA domain of Ubiquilin1 binds non-preferentially to different ubiquitin linkages, Ubiquilin1 dimerizes through its STI-4 domains and Ubiquilin1 continues to interact with its substrates in its monomeric and dimerized forms (Kurlawala et al., 2017).

\section{Strengths of this Dissertation}

Ubiquilin1 was discovered in 1999, followed by its other family members. UBQLN proteins are evolutionary conserved and have distinct tissue distribution therefore these proteins seem to be important from an evolutionary standpoint. We developed constructs of UBQLN1 to study functions of individual domains of the protein and their general role upon interaction with substrate. We tried to identify patterns in UBQLN1-substrate interaction based on literature and our data and classified substrates of UBQLN1 into 2 categories - stabilizers and degraders, thus helping us understand its mechanism of action. We demonstrated that Ubiquilin1 may be a key regulator of transmembrane proteins like BCLb, IGF1R, INSR, ESYT2 and EGFR and potentially influence their activity.

\section{Limitations of this Dissertation}

We did not test whether interaction of UBQLN1 with its substrates was a direct or indirect interaction. Irrespective of the type of interaction, UBQLN1 continues to regulate BCLb, ESYT2, and receptor tyrosine kinases we tested. Next, the domain deletion constructs of UBQLN1 may have altered secondary and tertiary structures and may affect 
binding with substrates. Although, theoretically changes in folding can affect binding, we continued to detect interactions for UBQLN1 with $\triangle \mathrm{STI}$ domain, $\triangle \mathrm{UBL}$ and $\triangle \mathrm{UBA}$ domains for the proteins we tested. Thirdly, we did not test for compensation by other UBQLN family members in cells that have loss of UBQLN1 function, which may be responsible for lack of phenotype in some cell types. Regulation of receptor tyrosine kinases were tested in a 2D in vitro model and cellular confluency is known to influence expression and activity of cell surface receptors. Therefore, regulation of receptor tyrosine kinases by UBQLN1 should be tested in a more relevant 3D model and in vivo.

\section{Future directions}

So far, we have only scratched the surface of understanding the mechanics of interaction between UBQLN1 and its substrates. Detailed experiments characterizing their regulation are warranted. For example, are cancers with loss of UBQLN1 and overexpression of IGF1R and EGFR more sensitive to IGFR and EGFR activity inhibition? ESYT proteins are calcium sensing proteins present at ER-plasma membrane contact sites and participate in connecting cellular compartments (Sclip, Bacaj, Giam, \& Sudhof, 2016) UBQLN1 interacts with several transmembrane proteins which directly or indirectly interact with cytoskeletal proteins like Vimentin (A. L. Wu et al., 1999). Does UBQLN1 act as a scaffold to hold protein complexes together and may also play a role in preserving normal cellular morphology and signaling networks? Identifying interactions of UBQLN1 with components of the membrane and cytoskeleton using co-immunoprecipitation analysis, crosslinking protein interaction assays for detection of transient interactions, visualization of contact sites and tracking changes in live cells via immunofluorescence and electron microscopy will be valuable. Previously, Ubiquilin2 has been shown to negatively regulate clathrin-mediated endocytosis of G Protein-Coupled Receptors (GPCR's) (N'Diaye et al., 2008). The UBL domain of Ubiquilin1 interacts with the UIMs 
(ubiquitin interacting motif) of EPS15, Hrs, Hbp (Regan-Klapisz et al., 2005). These proteins form a multi-complex and assist in sorting of ubiquitinated cargo (endocytosed EGF receptor) into multi-vesicular bodies (Gucwa \& Brown, 2014). Whether Ubiquilin1 participates in a similar complex for regulation of IGF1R and EGFR is not known. As IGF1R is involved in processes of normal growth, development, metabolism and even cancer progression, understanding its regulation by Ubiquilin 1 can be of tremendous value to many disciplines.

\section{Conclusions}

We identified 5 new transmembrane proteins as substrates of UBQLN1: ESYT2, IGF1R, IGF2R, INSR and EGFR. We proposed a new substrate stabilization model such that these proteins primarily interact with UBQLN1 through its STI domains and are stabilized by a secondary association via the UBA domain (tested only for BCLb, ESYT2, IGF1R). UBL-mediated interactions with BAG6 and PSMD4 do not result in their stabilization.

Using these data, we determined that UBQLN1 is essential in regulation of IGF1R in lung adenocarcinoma cells. Loss of UBQLN1 leads to accelerated loss of IGF1R. Loss of UBQLN1 leads to increased activation of the IGF pathway leading to increased survival and migration of these cells. Additionally, we found that UBQLN1 also interacts with Insulin receptor, IGF2R and EGFR.

Overall, we conclude that Ubiquilin1 performs a variety of functions in cells and fate of substrates it binds to is interaction-domain specific. Individual domains may determine Ubiquilin1's underlying mechanism of action and elucidate its role in neurodegenerative disorders as well as in cancers. Ubiquilin1 may play an important role in endocytosis and trafficking of proteins. Whether this role is via its UBA domain associating with ubiquitin on substrates or through one of its other domains remains to be investigated. 


\section{REFERENCES}

Adhikari, A., \& Chen, Z. J. (2009). Diversity of polyubiquitin chains. Dev Cell, 16(4), 485486. doi:10.1016/j.devcel.2009.04.001

Bedford, F. K., Kittler, J. T., Muller, E., Thomas, P., Uren, J. M., Merlo, D., . . Moss, S. J. (2001). GABA(A) receptor cell surface number and subunit stability are regulated by the ubiquitin-like protein Plic-1. Nat Neurosci, 4(9), 908-916. doi:10.1038/nn0901-908

Bertram, L., Hiltunen, M., Parkinson, M., Ingelsson, M., Lange, C., Ramasamy, K., . . . Tanzi, R. E. (2005). Family-based association between Alzheimer's disease and variants in UBQLN1. N Engl J Med, 352(9), 884-894. doi:10.1056/NEJMoa042765

Beverly, L. J., Lockwood, W. W., Shah, P. P., Erdjument-Bromage, H., \& Varmus, H. (2012). Ubiquitination, localization, and stability of an anti-apoptotic BCL2-like protein, BCL2L10/BCLb, are regulated by Ubiquilin1. Proc Natl Acad Sci U S A, 109(3), E119-126. doi:10.1073/pnas.1119167109

Brahmkhatri, V. P., Prasanna, C., \& Atreya, H. S. (2015). Insulin-like growth factor system in cancer: novel targeted therapies. Biomed Res Int, 2015, 538019. doi: $10.1155 / 2015 / 538019$

Buchberger, A. (2002). From UBA to UBX: new words in the ubiquitin vocabulary. Trends Cell Biol, 12(5), 216-221.

Buckley, D. A., Cheng, A., Kiely, P. A., Tremblay, M. L., \& O'Connor, R. (2002). Regulation of insulin-like growth factor type I (IGF-I) receptor kinase activity by protein tyrosine phosphatase 1B (PTP-1B) and enhanced IGF-I-mediated suppression of apoptosis and motility in PTP-1B-deficient fibroblasts. Mol Cell Biol, 22(7), 19982010.

Chen, G., Wang, X., Yu, J., Varambally, S., Yu, J., Thomas, D. G., . . Chinnaiyan, A. M. (2007). Autoantibody profiles reveal ubiquilin 1 as a humoral immune response target in lung adenocarcinoma. Cancer Res, 67(7), 3461-3467. doi:10.1158/00085472.CAN-06-4475

Clemmons, D. R., \& Maile, L. A. (2003). Minireview: Integral membrane proteins that function coordinately with the insulin-like growth factor I receptor to regulate intracellular signaling. Endocrinology, 144(5), 1664-1670. doi:10.1210/en.2002221102

Cohen, P., Graves, H. C., Peehl, D. M., Kamarei, M., Giudice, L. C., \& Rosenfeld, R. G. (1992). Prostate-specific antigen (PSA) is an insulin-like growth factor binding protein-3 protease found in seminal plasma. J Clin Endocrinol Metab, 75(4), 10461053. doi:10.1210/jcem.75.4.1383255

Cox, O. T., O'Shea, S., Tresse, E., Bustamante-Garrido, M., Kiran-Deevi, R., \& O'Connor, R. (2015). IGF-1 Receptor and Adhesion Signaling: An Important Axis in Determining Cancer Cell Phenotype and Therapy Resistance. Front Endocrinol (Lausanne), 6, 106. doi:10.3389/fendo.2015.00106

Datta, S. R., Dudek, H., Tao, X., Masters, S., Fu, H., Gotoh, Y., \& Greenberg, M. E. (1997). Akt phosphorylation of BAD couples survival signals to the cell-intrinsic death machinery. Cell, 91(2), 231-241. 
Deng, H. X., Chen, W., Hong, S. T., Boycott, K. M., Gorrie, G. H., Siddique, N., . . . Siddique, T. (2011). Mutations in UBQLN2 cause dominant X-linked juvenile and adult-onset ALS and ALS/dementia. Nature, 477(7363), 211-215. doi:10.1038/nature10353

Dufresne, A. M., \& Smith, R. J. (2005). The adapter protein GRB10 is an endogenous negative regulator of insulin-like growth factor signaling. Endocrinology, 146(10), 4399-4409. doi:10.1210/en.2005-0150

Dunn, S. E., Torres, J. V., Oh, J. S., Cykert, D. M., \& Barrett, J. C. (2001). Up-regulation of urokinase-type plasminogen activator by insulin-like growth factor-I depends upon phosphatidylinositol-3 kinase and mitogen-activated protein kinase kinase. Cancer Res, 61(4), 1367-1374.

Feng, P., Scott, C. W., Cho, N. H., Nakamura, H., Chung, Y. H., Monteiro, M. J., \& Jung, J. U. (2004). Kaposi's sarcoma-associated herpesvirus K7 protein targets a ubiquitin-like/ubiquitin-associated domain-containing protein to promote protein degradation. Mol Cell Biol, 24(9), 3938-3948.

Ficklin, M. B., Zhao, S., \& Feng, G. (2005). Ubiquilin-1 regulates nicotine-induced upregulation of neuronal nicotinic acetylcholine receptors. J Biol Chem, 280(40), 34088-34095. doi:10.1074/jbc.M506781200

Ford, D. L., \& Monteiro, M. J. (2007). Studies of the role of ubiquitination in the interaction of ubiquilin with the loop and carboxyl terminal regions of presenilin-2. Biochemistry, 46(30), 8827-8837. doi:10.1021/bi700604q

Gao, L., Tu, H., Shi, S. T., Lee, K. J., Asanaka, M., Hwang, S. B., \& Lai, M. M. (2003). Interaction with a ubiquitin-like protein enhances the ubiquitination and degradation of hepatitis C virus RNA-dependent RNA polymerase. J Virol, 77(7), 4149-4159.

Giovannone, B., Tsiaras, W. G., de la Monte, S., Klysik, J., Lautier, C., Karashchuk, G., . Smith, R. J. (2009). GIGYF2 gene disruption in mice results in neurodegeneration and altered insulin-like growth factor signaling. Hum $\mathrm{Mol}$ Genet, 18(23), 4629-4639. doi:10.1093/hmg/ddp430

Girnita, L., Girnita, A., \& Larsson, O. (2003). Mdm2-dependent ubiquitination and degradation of the insulin-like growth factor 1 receptor. Proc Natl Acad Sci U S A, 100(14), 8247-8252. doi:10.1073/pnas.1431613100

Girnita, L., Shenoy, S. K., Sehat, B., Vasilcanu, R., Girnita, A., Lefkowitz, R. J., \& Larsson, O. (2005). \{beta\}-Arrestin is crucial for ubiquitination and down-regulation of the insulin-like growth factor-1 receptor by acting as adaptor for the MDM2 E3 ligase. J Biol Chem, 280(26), 24412-24419. doi:10.1074/jbc.M501129200

Girnita, L., Worrall, C., Takahashi, S. G., 2014 \#72\}., Seregard, S., \& Girnita, A. (2014). Something old, something new and something borrowed: emerging paradigm of insulin-like growth factor type 1 receptor (IGF-1R) signaling regulation. Cell $\mathrm{Mol}$ Life Sci, 71(13), 2403-2427. doi:10.1007/s00018-013-1514-y

Goh, L. K., \& Sorkin, A. (2013). Endocytosis of receptor tyrosine kinases. Cold Spring Harb Perspect Biol, 5(5), a017459. doi:10.1101/cshperspect.a017459

Grimberg, A. (2003). Mechanisms by which IGF-I may promote cancer. Cancer Biol Ther, 2(6), 630-635.

Gucwa, A. L., \& Brown, D. A. (2014). UIM domain-dependent recruitment of the endocytic adaptor protein Eps15 to ubiquitin-enriched endosomes. BMC Cell Biol, 15, 34. doi:10.1186/1471-2121-15-34

Guidi, F., Puglia, M., Gabbiani, C., Landini, I., Gamberi, T., Fregona, D., . . Messori, L. (2012). 2D-DIGE analysis of ovarian cancer cell responses to cytotoxic gold compounds. Mol Biosyst, 8(4), 985-993. doi:10.1039/c1mb05386h 
Haugsten, E. M., Malecki, J., Bjorklund, S. M., Olsnes, S., \& Wesche, J. (2008). Ubiquitination of fibroblast growth factor receptor 1 is required for its intracellular sorting but not for its endocytosis. Mol Biol Cell, 19(8), 3390-3403. doi:10.1091/mbc.E07-12-1219

Heir, R., Ablasou, C., Dumontier, E., Elliott, M., Fagotto-Kaufmann, C., \& Bedford, F. K. (2006). The UBL domain of PLIC-1 regulates aggresome formation. EMBO Rep, 7(12), 1252-1258. doi:10.1038/sj.embor.7400823

Hjerpe, R., Bett, J. S., Keuss, M. J., Solovyova, A., McWilliams, T. G., Johnson, C., . . Wightman, M. (2016). UBQLN2 Mediates Autophagy-Independent Protein Aggregate Clearance by the Proteasome. Cell, 166(4), 935-949.

Hochrainer, K., Kroismayr, R., Baranyi, U., Binder, B. R., \& Lipp, J. (2008). Highly homologous HERC proteins localize to endosomes and exhibit specific interactions with hPLIC and Nm23B. Cell Mol Life Sci, 65(13), 2105-2117. doi:10.1007/s00018-008-8148-5

Hochrainer, K., Pejanovic, N., Olaseun, V. A., Zhang, S., ladecola, C., \& Anrather, J. (2015). The ubiquitin ligase HERC3 attenuates NF-kappaB-dependent transcription independently of its enzymatic activity by delivering the RelA subunit for degradation. Nucleic Acids Res, 43(20), 9889-9904. doi:10.1093/nar/gkv1064

Hoffrogge, R., Mikkat, S., Scharf, C., Beyer, S., Christoph, H., Pahnke, J., . . Rolfs, A. (2006). 2-DE proteome analysis of a proliferating and differentiating human neuronal stem cell line (ReNcell VM). Proteomics, 6(6), 1833-1847. doi:10.1002/pmic.200500556

Huang, F., Kirkpatrick, D., Jiang, X., Gygi, S., \& Sorkin, A. (2006). Differential regulation of EGF receptor internalization and degradation by multiubiquitination within the kinase domain. Mol Cell, 21(6), 737-748. doi:10.1016/j.molcel.2006.02.018

Itakura, E., Zavodszky, E., Shao, S., Wohlever, M. L., Keenan, R. J., \& Hegde, R. S. (2016). Ubiquilins Chaperone and Triage mitochondrial membrane proteins for degradation. Molecular Cell, 63(1), 21-33.

Janssen, J. A., \& Varewijck, A. J. (2014). IGF-IR Targeted Therapy: Past, Present and Future. Front Endocrinol (Lausanne), 5, 224. doi:10.3389/fendo.2014.00224

Jean, S., Mikryukov, A., Tremblay, M. G., Baril, J., Guillou, F., Bellenfant, S., \& Moss, T. (2010). Extended-synaptotagmin-2 mediates FGF receptor endocytosis and ERK activation in vivo. Dev Cell, 19(3), 426-439. doi:10.1016/j.devcel.2010.08.007

Jeong, S. J., Pise-Masison, C. A., Radonovich, M. F., Park, H. U., \& Brady, J. N. (2005). Activated AKT regulates NF-kappaB activation, p53 inhibition and cell survival in HTLV-1-transformed cells. Oncogene, 24(44), 6719-6728. doi:10.1038/sj.onc.1208825

Kleijnen, M. F., Shih, A. H., Zhou, P., Kumar, S., Soccio, R. E., Kedersha, N. L., . . . Howley, P. M. (2000). The hPLIC proteins may provide a link between the ubiquitination machinery and the proteasome. Molecular Cell, 6(2), 409-419.

Ko, H. S., Uehara, T., \& Nomura, Y. (2002). Role of ubiquilin associated with proteindisulfide isomerase in the endoplasmic reticulum in stress-induced apoptotic cell death. J Biol Chem, 277(38), 35386-35392. doi:10.1074/jbc.M203412200

Ko, H. S., Uehara, T., Tsuruma, K., \& Nomura, Y. (2004). Ubiquilin interacts with ubiquitylated proteins and proteasome through its ubiquitin-associated and ubiquitin-like domains. FEBS Lett, 566(1-3), 110-114. doi:10.1016/j.febslet.2004.04.031

Kornilova, E., Sorkina, T., Beguinot, L., \& Sorkin, A. (1996). Lysosomal targeting of epidermal growth factor receptors via a kinase-dependent pathway is mediated by the receptor carboxyl-terminal residues 1022-1123. J Biol Chem, 271(48), 3034030346. 
Kurlawala, Z., Shah, P. P., Shah, C., \& Beverly, L. J. (2017). The STI and UBA Domains of UBQLN1 are Critical Determinants of Substrate Interaction and Proteostasis. $J$ Cell Biochem. doi:10.1002/jcb. 25880

Langlais, P., Dong, L. Q., Ramos, F. J., Hu, D., Li, Y., Quon, M. J., \& Liu, F. (2004). Negative regulation of insulin-stimulated mitogen-activated protein kinase signaling by Grb10. Mol Endocrinol, 18(2), 350-358. doi:10.1210/me.2003-0117

Laron, Z. (2008). The GH-IGF1 axis and longevity. The paradigm of IGF1 deficiency. Hormones (Athens), 7(1), 24-27.

Lee, D. Y., Arnott, D., \& Brown, E. J. (2013). Ubiquilin4 is an adaptor protein that recruits Ubiquilin1 to the autophagy machinery. EMBO reports, 14(4), 373-381.

Lim, P. J., Danner, R., Liang, J., Doong, H., Harman, C., Srinivasan, D., . . Monteiro, M. J. (2009). Ubiquilin and p97/VCP bind erasin, forming a complex involved in ERAD. J Cell Biol, 187(2), 201-217. doi:10.1083/jcb.200903024

Lu, A., Hiltunen, M., Romano, D. M., Soininen, H., Hyman, B. T., Bertram, L., \& Tanzi, R. E. (2009). Effects of ubiquilin 1 on the unfolded protein response. J Mol Neurosci, 38(1), 19-30. doi:10.1007/s12031-008-9155-6

Madshus, I. H., \& Stang, E. (2009). Internalization and intracellular sorting of the EGF receptor: a model for understanding the mechanisms of receptor trafficking. J Cell Sci, 122(Pt 19), 3433-3439. doi:10.1242/jcs.050260

Mah, A. L., Perry, G., Smith, M. A., \& Monteiro, M. J. (2000). Identification of ubiquilin, a novel presenilin interactor that increases presenilin protein accumulation. $J$ Cell Biol, 151(4), 847-862.

Mao, Y., Shang, Y., Pham, V. C., Ernst, J. A., Lill, J. R., Scales, S. J., \& Zha, J. (2011). Polyubiquitination of insulin-like growth factor I receptor (IGF-IR) activation loop promotes antibody-induced receptor internalization and down-regulation. $J$ Biol Chem, 286(48), 41852-41861. doi:10.1074/jbc.M111.288514

Massey, L. K., Mah, A. L., Ford, D. L., Miller, J., Liang, J., Doong, H., \& Monteiro, M. J. (2004). Overexpression of ubiquilin decreases ubiquitination and degradation of presenilin proteins. J Alzheimers Dis, 6(1), 79-92.

Massey, L. K., Mah, A. L., \& Monteiro, M. J. (2005). Ubiquilin regulates presenilin endoproteolysis and modulates gamma-secretase components, Pen-2 and nicastrin. Biochem J, 391(Pt 3), 513-525. doi:10.1042/BJ20050491

Medicherla, B., Kostova, Z., Schaefer, A., \& Wolf, D. H. (2004). A genomic screen identifies Dsk2p and Rad23p as essential components of ER-associated degradation. EMBO Rep, 5(7), 692-697. doi:10.1038/sj.embor.7400164

Min, S.-W., Chang, W.-P., \& Südhof, T. C. (2007). E-Syts, a family of membranous Ca2+sensor proteins with multiple C2 domains. Proceedings of the National Academy of Sciences, 104(10), 3823-3828.

Mori, K., Giovannone, B., \& Smith, R. J. (2005). Distinct Grb10 domain requirements for effects on glucose uptake and insulin signaling. Mol Cell Endocrinol, 230(1-2), 3950. doi:10.1016/j.mce.2004.11.004

Mueller, T. D., Kamionka, M., \& Feigon, J. (2004). Specificity of the interaction between ubiquitin-associated domains and ubiquitin. J Biol Chem, 279(12), 11926-11936. doi:10.1074/jbc.M312865200

Muller-Vahl, K. R., Kolbe, H., \& Dengler, R. (1997). [Gilles de la Tourette syndrome. Effect of nicotine, alcohol and marihuana on clinical symptoms]. Nervenarzt, 68(12), 985989.

N'Diaye, E. N., Hanyaloglu, A. C., Kajihara, K. K., Puthenveedu, M. A., Wu, P., von Zastrow, M., \& Brown, E. J. (2008). The ubiquitin-like protein PLIC-2 is a negative regulator of $G$ protein-coupled receptor endocytosis. Mol Biol Cell, 19(3), 12521260. doi:10.1091/mbc.E07-08-0775 
N'Diaye, E. N., Kajihara, K. K., Hsieh, I., Morisaki, H., Debnath, J., \& Brown, E. J. (2009). PLIC proteins or ubiquilins regulate autophagy-dependent cell survival during nutrient starvation. EMBO Rep, 10(2), 173-179. doi:10.1038/embor.2008.238

N'Diaye, E. N., Kajihara, K. K., Hsieh, I., Morisaki, H., Debnath, J., \& Brown, E. J. (2009). PLIC proteins or ubiquilins regulate autophagy - dependent cell survival during nutrient starvation. EMBO reports, 10(2), 173-179.

Nakagawa, M., Uramoto, H., Oka, S., Chikaishi, Y., Iwanami, T., Shimokawa, H., . . . Tanaka, F. (2012). Clinical significance of IGF1R expression in non-small-cell lung cancer. Clin Lung Cancer, 13(2), 136-142. doi:10.1016/j.cllc.2011.10.006

Ozaki, T., Kondo, K., Nakamura, Y., Ichimiya, S., Nakagawara, A., \& Sakiyama, S. (1997). Interaction of DA41, a DAN-binding protein, with the epidermal growth factor-like protein, S(1-5). Biochem Biophys Res Commun, 237(2), 245-250. doi:10.1006/bbrc.1997.7122

Ozaki, T., Nakamura, Y., Hanaoka, E., Nakagawara, A., \& Sakiyama, S. (2000). Overexpression of DA41 in v-Ha-ras-3Y1 cells causes growth suppression. Jpn J Cancer Res, 91(10), 987-993.

Persson, P., Stockhausen, M. T., Pahlman, S., \& Axelson, H. (2004). Ubiquilin-1 is a novel $\mathrm{HASH}-1$-complexing protein that regulates levels of neuronal bHLH transcription factors in human neuroblastoma cells. Int $J$ Oncol, 25(5), 1213-1221.

Peschard, P., Fournier, T. M., Lamorte, L., Naujokas, M. A., Band, H., Langdon, W. Y., \& Park, M. (2001). Mutation of the c-Cbl TKB domain binding site on the Met receptor tyrosine kinase converts it into a transforming protein. Mol Cell, 8(5), 995-1004.

Ramos, F. J., Langlais, P. R., Hu, D., Dong, L. Q., \& Liu, F. (2006). Grb10 mediates insulinstimulated degradation of the insulin receptor: a mechanism of negative regulation. Am J Physiol Endocrinol Metab, 290(6), E1262-1266. doi:10.1152/ajpendo.00609.2005

Regan-Klapisz, E., Sorokina, I., Voortman, J., de Keizer, P., Roovers, R. C., Verheesen, P., . . . van Bergen en Henegouwen, P. M. (2005). Ubiquilin recruits Eps15 into ubiquitin-rich cytoplasmic aggregates via a UIM-UBL interaction. J Cell Sci, 118(Pt 19), 4437-4450. doi:10.1242/jcs.02571

Renehan, A. G., O'Connell, J., O'Halloran, D., Shanahan, F., Potten, C. S., O'Dwyer, S. T., \& Shalet, S. M. (2003). Acromegaly and colorectal cancer: a comprehensive review of epidemiology, biological mechanisms, and clinical implications. Horm Metab Res, 35(11-12), 712-725. doi:10.1055/s-2004-814150

Riley, B. E., \& Orr, H. T. (2006). Polyglutamine neurodegenerative diseases and regulation of transcription: assembling the puzzle. Genes Dev, 20(16), 2183-2192. doi:10.1101/gad.1436506

Rocchi, S., Tartare-Deckert, S., Sawka-Verhelle, D., Gamha, A., \& van Obberghen, E. (1996). Interaction of SH2-containing protein tyrosine phosphatase 2 with the insulin receptor and the insulin-like growth factor-I receptor: studies of the domains involved using the yeast two-hybrid system. Endocrinology, 137(11), 4944-4952. doi:10.1210/endo.137.11.8895367

Rothenberg, C., Srinivasan, D., Mah, L., Kaushik, S., Peterhoff, C. M., Ugolino, J., . . Monteiro, M. J. (2010). Ubiquilin functions in autophagy and is degraded by chaperone-mediated autophagy. Hum Mol Genet, 19(16), 3219-3232. doi:10.1093/hmg/ddq231

Sakowski, E. T., Koster, S., Portal Celhay, C., Park, H. S., Shrestha, E., Hetzenecker, S. E., . . Philips, J. A. (2015). Ubiquilin 1 Promotes IFN-gamma-Induced Xenophagy of Mycobacterium tuberculosis. PLoS Pathog, 11(7), e1005076. doi:10.1371/journal.ppat.1005076 
Saliba, R. S., Pangalos, M., \& Moss, S. J. (2008). The ubiquitin-like protein Plic-1 enhances the membrane insertion of GABAA receptors by increasing their stability within the endoplasmic reticulum. J Biol Chem, 283(27), 18538-18544. doi:10.1074/jbc.M802077200

Sclip, A., Bacaj, T., Giam, L. R., \& Sudhof, T. C. (2016). Extended Synaptotagmin (ESyt) Triple Knock-Out Mice Are Viable and Fertile without Obvious Endoplasmic Reticulum Dysfunction. PLoS One, 11(6), e0158295. doi:10.1371/journal.pone.0158295

Sehat, B., Andersson, S., Girnita, L., \& Larsson, O. (2008). Identification of c-Cbl as a new ligase for insulin-like growth factor-I receptor with distinct roles from Mdm2 in receptor ubiquitination and endocytosis. Cancer Res, 68(14), 5669-5677. doi:10.1158/0008-5472.CAN-07-6364

Sell, C., Rubini, M., Rubin, R., Liu, J. P., Efstratiadis, A., \& Baserga, R. (1993). Simian virus 40 large tumor antigen is unable to transform mouse embryonic fibroblasts lacking type 1 insulin-like growth factor receptor. Proc Natl Acad Sci U S A, 90(23), 11217-11221.

Shah, P. P., Lockwood, W. W., Saurabh, K., Kurlawala, Z., Shannon, S. P., Waigel, S., . . . Beverly, L. J. (2015). Ubiquilin1 represses migration and epithelial-tomesenchymal transition of human non-small cell lung cancer cells. Oncogene, 34(13), 1709-1717. doi:10.1038/onc.2014.97

Shimada, K., Fujii, T., Tatsumi, Y., Anai, S., Fujimoto, K., \& Konishi, N. (2016). Ubiquilin2 as a novel marker for detection of urothelial carcinoma cells in urine. Diagn Cytopathol, 44(1), 3-9. doi:10.1002/dc.23332

Stein, E. G., Gustafson, T. A., \& Hubbard, S. R. (2001). The BPS domain of Grb10 inhibits the catalytic activity of the insulin and IGF1 receptors. FEBS Lett, 493(2-3), 106111.

Sun, Q., Liu, T., Yuan, Y., Guo, Z., Xie, G., Du, S., . . Chen, L. (2015). MiR-200c inhibits autophagy and enhances radiosensitivity in breast cancer cells by targeting UBQLN1. Int J Cancer, 136(5), 1003-1012. doi:10.1002/ijc.29065

Tanzi, R. E., \& Bertram, L. (2005). Twenty years of the Alzheimer's disease amyloid hypothesis: a genetic perspective. Cell, 120(4), 545-555. doi:10.1016/j.cell.2005.02.008

Tremblay, M. G., Herdman, C., Guillou, F., Mishra, P. K., Baril, J., Bellenfant, S., \& Moss, T. (2015). Extended Synaptotagmin Interaction with the Fibroblast Growth Factor Receptor Depends on Receptor Conformation, Not Catalytic Activity. J Biol Chem, 290(26), 16142-16156. doi:10.1074/jbc.M115.656918

Tsukamoto, S., Shimada, K., Honoki, K., Kido, A., Akahane, M., Tanaka, Y., \& Konishi, N. (2015). Ubiquilin 2 enhances osteosarcoma progression through resistance to hypoxic stress. Oncol Rep, 33(4), 1799-1806. doi:10.3892/or.2015.3788

Varadan, R., Assfalg, M., Haririnia, A., Raasi, S., Pickart, C., \& Fushman, D. (2004). Solution conformation of Lys63-linked di-ubiquitin chain provides clues to functional diversity of polyubiquitin signaling. J Biol Chem, 279(8), 7055-7063. doi:10.1074/jbc.M309184200

Vecchione, A., Marchese, A., Henry, P., Rotin, D., \& Morrione, A. (2003). The Grb10/Nedd4 complex regulates ligand-induced ubiquitination and stability of the insulin-like growth factor I receptor. Mol Cell Biol, 23(9), 3363-3372.

Vigneri, P. G., Tirro, E., Pennisi, M. S., Massimino, M., Stella, S., Romano, C., \& Manzella, L. (2015). The Insulin/IGF System in Colorectal Cancer Development and Resistance to Therapy. Front Oncol, 5, 230. doi:10.3389/fonc.2015.00230

Wang, F., Duan, R., Chirgwin, J., \& Safe, S. H. (2000). Transcriptional activation of cathepsin D gene expression by growth factors. J Mol Endocrinol, 24(2), 193-202. 
Wang, H., \& Monteiro, M. J. (2007). Ubiquilin interacts and enhances the degradation of expanded-polyglutamine proteins. Biochem Biophys Res Commun, 360(2), 423427. doi:10.1016/j.bbrc.2007.06.097

Wang, L. M., Myers, M. G., Jr., Sun, X. J., Aaronson, S. A., White, M., \& Pierce, J. H. (1993). IRS-1: essential for insulin- and IL-4-stimulated mitogenesis in hematopoietic cells. Science, 261(5128), 1591-1594.

Wilkinson, C. R., Seeger, M., Hartmann-Petersen, R., Stone, M., Wallace, M., Semple, C., \& Gordon, C. (2001). Proteins containing the UBA domain are able to bind to multiubiquitin chains. Nat Cell Biol, 3(10), 939-943. doi:10.1038/ncb1001-939

Wills, M. K., \& Jones, N. (2012). Teaching an old dogma new tricks: twenty years of Shc adaptor signalling. Biochem J, 447(1), 1-16. doi:10.1042/BJ20120769

Wu, A. L., Wang, J., Zheleznyak, A., \& Brown, E. J. (1999). Ubiquitin-related proteins regulate interaction of vimentin intermediate filaments with the plasma membrane. Mol Cell, 4(4), 619-625.

Wu, S., Mikhailov, A., Kallo-Hosein, H., Hara, K., Yonezawa, K., \& Avruch, J. (2002). Characterization of ubiquilin 1, an mTOR-interacting protein. Biochim Biophys Acta, 1542(1-3), 41-56.

Yadav, S., Singh, N., Shah, P. P., Rowbotham, D. A., Malik, D., Srivastav, A., . . Beverly, L. J. (2017). MIR155 Regulation of Ubiquilin1 and Ubiquilin2: Implications in Cellular Protection and Tumorigenesis. Neoplasia, 19(4), 321-332. doi:10.1016/j.neo.2017.02.001

Yarden, Y. (2001). The EGFR family and its ligands in human cancer. signalling mechanisms and therapeutic opportunities. Eur J Cancer, 37 Suppl 4, S3-8.

Yoon, A., \& Hurta, R. A. (2001). Insulin like growth factor-1 selectively regulates the expression of matrix metalloproteinase- 2 in malignant $\mathrm{H}$-ras transformed cells. $\mathrm{Mol}$ Cell Biochem, 223(1-2), 1-6.

Zhang, C., \& Saunders, A. J. (2009). An emerging role for Ubiquilin 1 in regulating protein quality control system and in disease pathogenesis. Discov Med, 8(40), 18-22.

Zhang, D., \& Brodt, P. (2003). Type 1 insulin-like growth factor regulates MT1-MMP synthesis and tumor invasion via PI 3-kinase/Akt signaling. Oncogene, 22(7), 974982. doi:10.1038/sj.onc.1206197

Zhang, D., Raasi, S., \& Fushman, D. (2008). Affinity makes the difference: nonselective interaction of the UBA domain of Ubiquilin-1 with monomeric ubiquitin and polyubiquitin chains. J Mol Biol, 377(1), 162-180. doi:10.1016/j.jmb.2007.12.029

Zhang, T., \& Jia, Y. (2014). Meta-analysis of Ubiquilin1 gene polymorphism and Alzheimer's disease risk. Med Sci Monit, 20, 2250-2255. doi:10.12659/MSM.891030

Zhang, Y., Li, Z., Gu, J., Zhang, Y., Wang, W., Shen, H., . . Wang, X. (2015). Plic-1, a new target in repressing epileptic seizure by regulation of GABAAR function in patients and a rat model of epilepsy. Clinical Science, 129(12), 1207-1223.

Zhao, L., \& Ackerman, S. L. (2006). Endoplasmic reticulum stress in health and disease. Curr Opin Cell Biol, 18(4), 444-452. doi:10.1016/j.ceb.2006.06.005

Zhao, S., Qiu, Z., He, J., Li, L., \& Li, W. (2014). Insulin-like growth factor receptor 1 (IGF1R) expression and survival in non-small cell lung cancer patients: a metaanalysis. Int J Clin Exp Pathol, 7(10), 6694-6704. 


\section{CURRICULUM VITAE}

Zimple Kurlawala M.D., M.P.H., M.S.

833 E Madison St., Apt F, Louisville, KY 40204

PHONE 270.392.9207•EMAIL zimple323@gmail.com

EDUCATION

2017 PhD in Pharmacology \& Toxicology, University of Louisville, KY (07/2017)

2015 Masters in Pharmacology \& Toxicology, University of Louisville, KY (08/2015) GPA: 3.72

2011 M.P.H., Western Kentucky University, Bowling Green, KY (01/2009 - 08/2011) GPA: 3.81

2008 M.D., University of Seychelles - American Institute of Medicine (USAIM), Victoria, Seychelles (08/2004 - 11/2008)

Honors: Internal Medicine, Family Medicine, Radiology, Ophthalmology, Medical Terminology

President of Student Body at USAIM (2004 - 2005)

U.S. CLINICAL EXPERIENCE

2012 Insured hands-on clinical experience supervised and evaluated by teaching attending physicians in a sub-internship-like atmosphere.

Psychiatry (4 weeks), PACT Atlanta, Decatur, GA (11/12)

Internal Medicine (4 weeks), Emory University Hospital Midtown, Atlanta, GA $(10 / 12$ - 10/12)

Neurology (4 weeks), Piedmont Hospital, Atlanta, GA (09/12 - 09/12)

Neurology (4 weeks), Piedmont Hospital, Fayetteville, GA (07-08/12)

Family Medicine (4 weeks), Cascade Primary Care, Atlanta, GA (07/12)

- Individual Performance Evaluations available

Geriatric Medicine (6 weeks), Observership, Life Care Centers of America, Palm Bay, FL (05-06/2012) 
- Kurlawala, Z \& Beverly, L. (2017) Ubiquilin Proteins are critical adaptors that regulate proteostasis. Journal of Cell Signaling 2:145

- Kurlawala, Z., Shah PP, Shah C, Beverly, L., (2017) The STI and UBA domains of UBQLN1 are critical determinants of substrate interaction and proteostasis. Journal of Cellular Biochemistry, 201. [PMID 28075048]

- Kurlawala, Z., \& Vatsalya, V. (2016). Heavy Alcohol Drinking Associated Akathisia and Management with Quetiapine XR in Alcohol Dependent Patients. Journal of Addiction, 2016. [PMID 27847671]

- Shah, P. P., Lockwood, W. W., Saurabh, K., Kurlawala, Z., Shannon, S. P., Waigel, S., ... \& Beverly, L. J. (2015). Ubiquilin1 represses migration and epithelialto-mesenchymal transition of human non-small cell lung cancer cells. Oncogene, 34(13), 1709-1717.

- SF, Jones GM, Jones ML, Kurlawala ZD, Leet TA, Lovell L.J, Phillips KE, Shoup LE. Complete Genome Sequences of 138 Mycobacteriophages. Journal of Virology. 2012 Feb; 86(4): 2382-2384. [PMID: 22282335]

IN PREPARATION

- Kurlawala, Z., Shah, P. P., Gosney, J., Siskind, L.J., Ceresa, B.P. \& Beverly, L. J. (2017). Regulation of Insulin Growth Factor Receptors by UBQLN1.

- Kurlawala, Z., \& Beverly, L. J. (2017). UBQLN1-The Multidomain Protein with Multiple Functions, Review.

- Kurlawala, Z., Shah, P. P., Gosney, J., Siskind, L.J., Ceresa, B.P. \& Beverly, L. J. (2017). Regulation of Epidermal Growth Factor Receptors by UBQLN1.

HONORS \& AWARDS

2017 Graduate Student Travel Award, American Society for Pharmacology and Experimental Therapeutics (ASPET) at Experimental Biology, Chicago, IL

2016 Reviewer for BMJ Global Health journal

2016 Arno Spatola Endowment Graduate Research Fellowship 
The Arno Spatola Graduate Research Fellowship is awarded to an exceptional graduate student who has received or is entering candidacy for the $\mathrm{Ph}$. D. degree under the guidance of an Institute for Molecular Diversity and Drug Design (IMD3) faculty member.

2016 First Place - Graduate Research

Graduate Student Council Regional Research Conference, University of Louisville.

2016 Candidacy Fee Award

School of Interdisciplinary Graduate Studies, University of Louisville.

2015 Graduate Student Ambassador, University of Louisville, KY

2015 Graduate Student Council Research Fund Award, University of Louisville, KY

2014 First Place - Masters Level-Basic Science Research

Research!Louisville, , Louisville, KY

2014 Institute for Molecular Diversity and Drug Design (IMD3) Travel Award, University of Louisville, KY

2013 Integrated Program in Biomedical Sciences Fellowship, University of Louisville, KY (2013-2015)

2012 Presenter, "Lunch and Learn" presentation on congestive heart failure for the nursing staff at Life Care Centers of America, Palm Bay, FL (06/2012)

2012 Athlete of the Week, Western Kentucky University

2010 Recipient of the American Humanics Emerging Non-Profit Award, 2010, as a participant in the Future Selves Program, Western Kentucky University (20072010)

2010 Best Student Chapter Award, served as Vice President of KPHA, Western Kentucky University

2010 Guest Lecturer, First Aid for Injury Emergencies, Bowling Green Housing Authorities

2006 Honors: Internal Medicine, Family Medicine, Radiology, Ophthalmology, Medical Terminology

2005 President of Student Body, USAIM

ORAL PRESENTATIONS

- Kurlawala, Z., Shah PP, Shah C, Beverly, L., (July 2016) The STI and UBA domains of UBQLN1 are critical determinants of substrate interaction and 
proteostasis, Cancer Colloquia, James Graham Brown Cancer Center, University of Louisville, $\mathrm{KY}$

- Kurlawala, Z., Beverly, L. (April 2016) Regulation of IGF1R by Ubiquilin1, Graduate Student Council Research Regional Symposium, Louisville, KY.

- Kurlawala, Z., Beverly, L. (Nov 2015) Regulation of IGF1R by Ubiquilin1, IMD3 Fall Fest, University of Louisville, KY.

- Kurlawala, Z., English, G. (2011). Examining college students' perceptions about organ donation, WKU Research Conference; Bowling Green, KY.

- Kurlawala, Z., Nagy C., English, G. (2011). Examining college students' perceptions about organ donation, Kentucky Public Health Association, Louisville, $\mathrm{KY}$.

- Kurlawala, Z., Nagy, S. (2010). A personalized programming approach towards sexual violence risk reduction, WKU Research Conference, Bowling Green, KY.

- Kurlawala, Z., Nagy, S. (2010). A personalized programming approach towards sexual violence risk reduction, Kentucky Public Health Association, Louisville, KY.

- Kurlawala, Z., Nagy, S. (2010). A personalized programming approach towards sexual violence risk reduction. American Public Health Association, Denver, CO.

\section{POSTER PRESENTATIONS}

- Kurlawala, Z., Shah PP, Shah C, Beverly, L., (Nov 2016) The STI and UBA domains of UBQLN1 are critical determinants of substrate interaction and proteostasis, Southeast Regional Lipid Conference, Cashiers, NC.

- Kurlawala, Z., Beverly, L., (Oct 2015) Understanding the role of UBQLN1 in lung cancer, Research!Louisville, Louisville, KY.

- Kurlawala, Z., Malik, D., Beverly, L., (Oct 2014) Regulation of IGF1R by Ubiquilin1, Midwest Membrane Trafficking and Signaling Symposia, American Society for Cell Biology, Louisville, KY.

- Kurlawala, Z., Malik, D., Beverly, L., (Aug 2014) Regulation of IGF1R by Ubiquilin1, Mechanisms and Models of Cancer, Cold Spring Harbor Laboratories, NY.

- Kurlawala, Z., Malik, D., Beverly, L., (Sept. 2014) Regulation of IGF1R by Ubiquilin1, Research!Louisville, Louisville, KY. 
- Kurlawala, Z., Nagy, C. (2011) Examining college students' perceptions about organ donation. Poster presented at: American Public Health Association, D.C.

WORK EXPERIENCE

2013 Graduate Student, Dept. of Pharmacology/Toxicology, University of Louisville, KY (08/2013--)

2012 Part-time Faculty, Department of Public Health, Western Kentucky University, Bowling Green, KY (06/2009 - 05/2012)

- Taught American Heart Association curriculum, 'Heartsaver First Aid and CPR'. Provided opportunity for students to obtain certification as CPR and first provider.

2012 Public Health Intern, Barren County Public Health Department, KY (01/2011 08/2011)

- Smoking coalition; prepared presentations on cardiovascular diseases and obesity which are now being used as health education materials in 8 counties in Kentucky; participated in school health fair for elementary school children, participated in a health education booth about CDC recommended food safety guidelines at Kroger grocery store in Bowling Green, KY

2009 Instructor, Juvenile Detention Center, Bowling Green, KY

2009 Master Control Operator

WKYU-PBS, Western Kentucky University, KY (06 - 08/2009)

CONFERENCES, WORKSHOPS AND SYMPOSIA

2017 American Society for Pharmacology and Experimental Therapeutics (ASPET) at Experimental Biology, Chicago, IL

2016 Southeastern Regional Lipid Conference, Cashiers, NC

2016 Kentucky Lung Cancer Symposium, Lexington, KY

2016 Research!Louisville, University of Louisville, KY

2016 Geoffrey P. Herzig Memorial Leukemia Symposium, Louisville, KY

2016 Graduate Student Council Regional Research Conference, Louisville, KY

2015 Research!Louisville, University of Louisville, KY 
2015 Institute of Molecular Diversity and Drug Design Fall Fest, Louisville, KY

2014 James Graham Brown Cancer Center Retreat, Louisville, KY

2014 Mechanisms and Models of Cancer, Cold Spring Harbor Laboratories, NY

2014 Research!Louisville, University of Louisville, KY

2011 American Public Health Association (APHA) Annual Conference, Washington, D.C. $(10 / 2011)$

Kentucky Public Health Association (KPHA) Annual Conference, Louisville, KY $(03 / 2011)$

Western Kentucky University Annual Research Conference, Bowling Green, KY (02/2011)

2010 APHA Annual Conference, Denver, CO (11/2010)

KPHA Annual Conference, Louisville, KY (03/2010)

WKU Annual Research Conference, Bowling Green, KY (02/2010) 